\title{
Existence theorems for noncoercive incremental contact problems with Coulomb friction
}

\author{
Andreas Rietz
}


Existence theorems for noncoercive incremental contact problems with Coulomb friction

Andreas Rietz

Department of Mathematics

Linköping University

SE-581 83 Linköping, Sweden

ISBN 91-85297-38-0

ISSN 0345-7524

Printed by UniTryck, Linköping 2005 


\section{Contents}

Abstract v v

Acknowledgements vii

I Introduction 1

1 Intent and motivation . . . . . . . . . . . . . 3

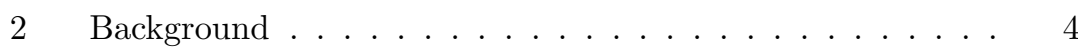

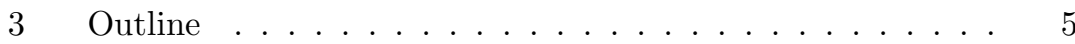

II Discrete Systems $\quad 7$

1 Preliminaries 9

1 Notation and problem formulation . . . . . . . . . 9

2 Existence results for discrete contact problems $\quad 15$

1 Existence of solutions in the coercive case . . . . . . . . 15

2 Existence of solutions in the noncoercive case . . . . . . . 17

3 Interpretation . . . . . . . . . . . . . . 21

4 Previous results . . . . . . . . . . . . . . . . 24

3 Examples of noncoercive friction problems $\quad 27$

1 Single node . . . . . . . . . . . . . . . . 27

2 Truss with three nodes . . . . . . . . . . . . . 28

3 Two dimensional truss on a flat obstacle . . . . . . . . . . . 29

4 Cylinder in a cylindrical groove. . . . . . . . . . . . 32

4 Corollaries from the existence theorem 39

1 Boundedness of the solution . . . . . . . . . . . 39

2 Stability results for the solution of two dimensional problems 40

3 Example on the stability results . . . . . . . . . . . 43 
III Continuous Systems with Nonlocal Friction Laws

5 Preliminaries $\quad \mathbf{4 7}$

1 Notation and problem formulation . . . . . . . . . . 47

2 Variational formulation . . . . . . . . . . . . . . 51

6 Existence results for nonlocal friction laws $\quad 57$

1 Existence theorem for three dimensional problems . . . . . 57

2 Previous results . . . . . . . . . . . . . . . 71

3 Appendix: Some elementary estimates . . . . . . . . . 72

7 Example on nonlocal friction $\quad \mathbf{7 7}$

1 Some auxiliary results . . . . . . . . . . . . . . 77

2 Example: Flat obstacle . . . . . . . . . . . . . . 78

\section{Continuous Systems with Normal Compliance} Friction Laws $\quad 83$

8 Preliminaries $\quad 85$

1 Notation and problem formulation . . . . . . . . 85

9 Existence results for normal compliance friction laws $\quad 91$

1 Auxiliary friction problem . . . . . . . . . . . . . 91

2 Existence results . . . . . . . . . . . . . . . . . 95

10 Extensions 103

1 Initial displacement . . . . . . . . . . . . . . . . . 103

2 Discrete friction problems with compliance friction laws . . 104

$\begin{array}{lll}\text { V Concluding remarks } & 107\end{array}$

1 Conclusions and future research . . . . . . . . . . . . 109

$\begin{array}{ll}\text { Bibliography } & 111\end{array}$ 


\section{Abstract}

Friction is a phenomenon which is present in most mechanical devices and frequently encountered in everyday life. In particular, understanding of this phenomenon is important in the modelling of contact between an elastic object and an obstacle. Noncoercive incremental contact problems with Coulomb friction constitute an important class of such friction problems due to their frequent occurrence in mechanical engineering. They occur for example when modelling an object which is not fixed to a support. The topic of this thesis is to study this class of friction problems.

This thesis considers both discrete and continuous systems. For the continuous systems we consider both problems with a nonlocal friction law where the contact force is mollified and problems with a normal compliance friction law where the body may penetrate the obstacle. For all friction problems we derive a sufficient condition for the existence of a solution. This condition is a compatibility condition on the applied force field, and if it is violated there exists a nontrivial solution to a corresponding dynamical problem. 


\section{Acknowledgements}

I would like to thank professor Lars-Erik Andersson for his supervision, for his encouragement and for providing a challenging topic of research. This work was a project of the graduate school Programme for Interdisciplinary Mathematics at the University of Linköping, and it is the outcome of a collaboration between the Department of Mathematics and the Department of Mechanical engineering. I thank all people involved, in particular professor Anders Klarbring for commenting on earlier manuscripts. In addition, my graduate studies have been supported by grants from the Swedish Research Council for engineering sciences (grant 621-2002-5693), the Royal Swedish Academy of Sciences, the foundation of G S Magnusson, the Danish Center for Applied Mathematics and Mechanics and the foundation of Knut and Alice Wallenberg. Moreover, the National Graduate School in Scientific Computing (NGSSC) admitted me to some of their graduate courses. I also thank prof. Marius Cocou at the Laboratoire de Mecanique et d'Acoustique, CNRS, Marseille, France, for his hospitality.

Andreas Rietz

Linköping, 6 March 2004 
Part I

\section{Introduction}





\section{Intent and motivation}

Noncoercive friction problems are frequently encountered in everyday life, for example when piercing a potato with a fork (see Figure 1). In this example, it is of interest to know whether or not the potato will slide off the plate when the fork touches its skin.

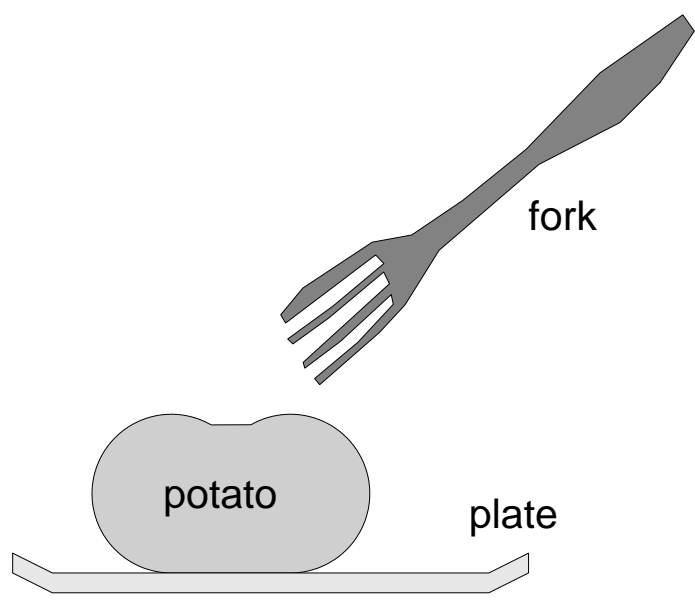

Figure 1: An example of a noncoercive friction problem: A fork piercing a potato.

To put this problem into a mathematical form, we require a mathematical model of the friction between the potato and the plate, a model of the composition of the potato and a knowledge about the shape of the potato and the plate. The object of this thesis is to study the mathematically posed noncoercive friction problem and, in particular, determine the existence of solutions of this problem. The reason is to gain a better understanding of the frictional problem and to use adequate models for e.g. numerical simulation.

There are many mechanical systems where it is important to analyse frictional contact. Examples include car breaks [33], railway wagon - rail contact [26], ball bearings and gears [27]. There are also geophysical applications such as the modelling of earthquakes [29].

Figure 2 is a mechanical illustration of the noncoercive problem in Figure 1. In mechanical terms, the potato is considered as an elastic body while the plate is considered as a rigid obstacle. The action of the fork is modelled by an applied force.

The problem formulation in this thesis is a model of the mechanical friction problem, and a few assumptions have been made. Firstly, the problem formulation neglects the acceleration of the body. This simplification is called a quasistatic assumption, and is used in many applications. 


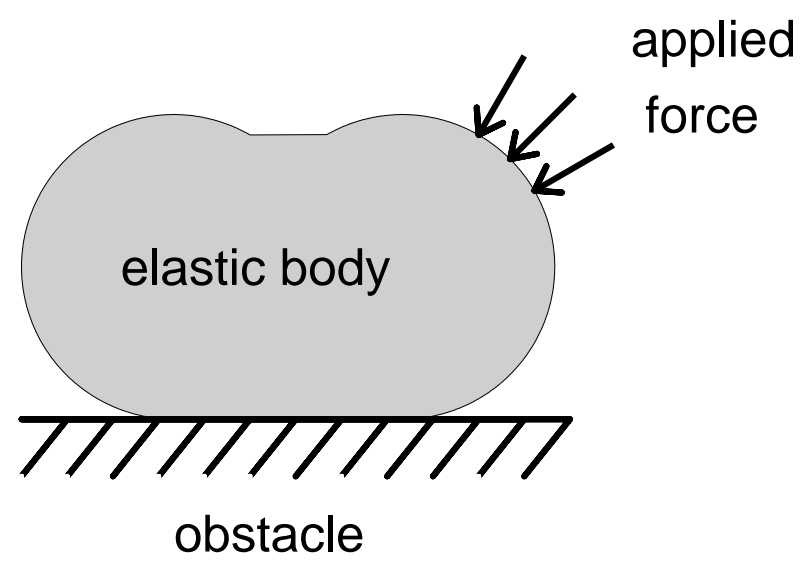

Figure 2: Mechanical model of a noncoercive friction problem.

Moreover, we use a Coulomb type of friction model, which means that the tangential frictional forces are proportional to the normal forces. Also, velocities are approximated by finite differences of the displacements. This thesis considers both discrete systems and continuous systems. Discrete systems refer for instance to trusses, where the displacement field can be represented by a finite number of variables. Continuous systems refer to continuous elastic bodies. Friction problems for discrete systems are called discrete friction problems and friction problems for continuous systems are called continuous friction problems.

We shall not prove any uniqueness of the solutions to the friction problems. In fact, even for simple elastic systems there can be multiple solutions provided the coefficient of friction is large, see [17], [18] and [23].

The problem illustrated in Figure 2 will recur as an example in this thesis. The topic in [4] is a similar example in three dimensions for a symmetric body and a symmetry condition on the applied force.

\section{Background}

Signorini's law of contact and Coulomb's law of friction are frequently used to model contact between bodies. Despite the simplicity of these laws, the existence of solutions to various contact problems involving them is still an open problem.

This thesis considers incremental friction problems, which means that velocities have been approximated by backward differences of successive displacements. Only a few existence theorems for friction problems with- 
out the incremental approximation have been presented, see for example Andersson [1], and the counterexamples by Klarbring [23] and Ballard [2] indicate that such theorems must put restrictive conditions on the applied force and the coefficient of friction.

The main issue of this thesis is to present existence theorems for noncoercive friction problems. In this thesis, noncoercivity means that the stiffness matrix is semidefinite in discrete friction problems and the bilinear elastic energy functional is noncoercive in continuous friction problems. For example we obtain a noncoercive friction problem when no displacements are prescribed, which occurs if the body is not fixed to a support. In case the stiffness matrix is definite or the bilinear elastic energy functional is coercive, we call the friction problem coercive.

The theory of coercive friction problems has been more developed than the theory of noncoercive friction problems, since some fixed point theorems and linear complementarity programming results can be used to study the coercive case. The first proof of existence of solutions to a general class of incremental coercive friction problems was due to Duvaut and Lion in 1972, [10] and [11]. However, they only established the existence of solutions to friction problems with prescribed normal pressure on the contact surface. Later, in 1980, Duvaut [9] studied another class of friction problems where the normal pressure is mollified. This regularization enabled him to prove the existence of solutions and, provided the coefficient of friction was small, also uniqueness. Since then, similar results have been proved by i.e. Demkowicz and Oden in 1982 [8] and Cocou in 1984 [6].

In 1980 Necas et. al. [28] proved an existence result for a friction problem without any regularization of the friction, but the treatment was restricted to an infinite strip in the plane and the coefficient of friction was assumed to be small. Jarušek [20] generalized the results in [28] to more general domains but the assumption on the coefficient of friction remained. The results were also generalized to the noncoercive case [21], but the outcome was a restrictive compatibility condition on the applied force that does not involve the coefficient of friction.

An analysis of noncoercive discrete systems with an incremental Coulomb friction model was performed by Klarbring and Pang in 1998 [25], using linear complementarity programming. Their results on existence of solutions to the friction problem are similar to some results in this thesis. This thesis interprets and polishes these conditions for existence of solutions, and hence make it possible to consider some applicable examples of noncoercive friction problems.

\section{Outline}

This thesis is divided in five parts, and each part is divided in chapters. Part I introduces the friction problem and part V summarizes the results. Part II, III and IV contain the main material. 
Part II: This part treats discrete systems. The main results are the theorem on the existence of solutions to the noncoercive friction problem, Theorem 2.3, and the examples of noncoercive friction problems in Chapter 3 . Chapter 1 introduces the notation and formulates the discrete incremental Coulomb friction problem. Chapter 2 presents results on the existence of solutions for discrete coercive and noncoercive friction problems. The proof of existence of solutions for the coercive friction problem applies the same methodology as Cocou [6], using fixed point theory originally developed by Fan [13] and Brezis et. al. [5]. This proof is included to make the thesis comprehensive. The noncoercive friction problem is analysed by considering an auxiliary coercive friction problem, in the same way as Klarbring and Pang [25]. In Chapter 3 results from Chapter 2 are applied to find sufficient conditions on the applied force that yield existence of solutions of some noncoercive friction problems. A single node on a flat obstacle is considered to illustrate the theory, and then an arbitrary truss on a flat obstacle and a truss in a cylindrical groove are considered to show the efficiency of the existence theorem. Finally, Chapter 4 contains some corollaries from the proof of Theorem 2.3.

Part III: This part treats noncoercive continuous systems with a nonlocal incremental Coulomb law of friction. Chapter 5 introduces the notation, presents the assumptions on the shape of the contact surface and establishes some mathematical properties of the problem. Then Chapter 6 considers the issue of existence of solutions to the friction problem with a nonlocal friction law. The focus is the three dimensional case, but the theorems given also apply in the two dimensional case with only small alterations. Theorems on the existence of solutions to the coercive friction problem are available in Cocou [6] and Kikuchi and Oden [22]. The existence of solutions of the noncoercive friction problem is possible to analyse by considering an auxiliary coercive problem. We derive conditions on the applied force that ensure the existence of solutions. Section 3 contains some basic estimates that are required in the proof. Finally, Chapter 7 considers an object on a flat obstacle as an example on how to apply the nonlocal friction laws.

Part IV: Chapter 8 formulates a friction problem with a normal compliance friction law. Notation and premises are stated, and constraints on the shape of the contact surface are imposed. A variational formulation is proved. Chapter 9 derives a sufficient condition for the existence of solutions to the problem. The condition is similar to the conditions in earlier chapters. In the end, Chapter 10 considers the effect of an initial gap and displacement. Moreover this chapter comments on the case of discrete friction problems with normal compliance friction laws. 


\section{Part II}

\section{Discrete Systems}





\section{Chapter 1}

\section{Preliminaries}

This chapter introduces the notation and formulates the discrete friction problem studied in this thesis.

\section{Notation and problem formulation}

Let us consider a truss consisting of $n$ nodes, compare Figure 1.1 where $n=6$. Some of these nodes can come into contact with a rigid obstacle. Denote the set of indices of these nodes by $I_{c}$.

Let $\overline{\mathbf{u}}_{i}$ be the displacement of node $i$. We form a block vector $\overline{\mathbf{u}}^{P}$ of the prescribed displacements and a block vector $\overline{\mathbf{u}}$ of the remaining displacements. A force $\overline{\mathbf{f}}_{i}$ act at each node $i$. We denote the block vector of forces corresponding to the nodes with prescribed displacements by $\overline{\mathbf{f}}^{P}$, and the block vector of the remaining forces by $\overline{\mathbf{f}}^{*}$. The linear elasticity of the truss is expressed by

$$
\left[\begin{array}{cc}
\mathbf{K}_{1} & \mathbf{K}_{2} \\
\mathbf{K}_{3} & \mathbf{K}
\end{array}\right]\left[\begin{array}{c}
\overline{\mathbf{u}}^{P} \\
\overline{\mathbf{u}}
\end{array}\right]=\left[\begin{array}{c}
\overline{\mathbf{f}}^{P} \\
\overline{\mathbf{f}}^{*}
\end{array}\right]
$$

if all displacements are measured from equilibrium. Thus, let us define $\overline{\mathbf{f}}$ by

$$
\mathbf{K} \overline{\mathbf{u}}=\overline{\mathbf{f}}^{*}-\mathbf{K}_{3} \overline{\mathbf{u}}^{P}=\overline{\mathbf{f}} .
$$

The stiffness matrix, $\mathbf{K}$, is symmetric and positive semidefinite. $\mathbf{K}$ is positive definite if a sufficiently large number of nodes have prescribed displacements, for example when the truss is rigidly anchored. Moreover, we decompose the force $\overline{\mathbf{f}}$ into a block vector of contact forces, $\mathbf{r}$, caused by the contact and a block vector of externally applied forces $\mathbf{f}^{a p p}$, so that $\overline{\mathbf{f}}=\mathbf{f}^{a p p}+\mathbf{r}$. 


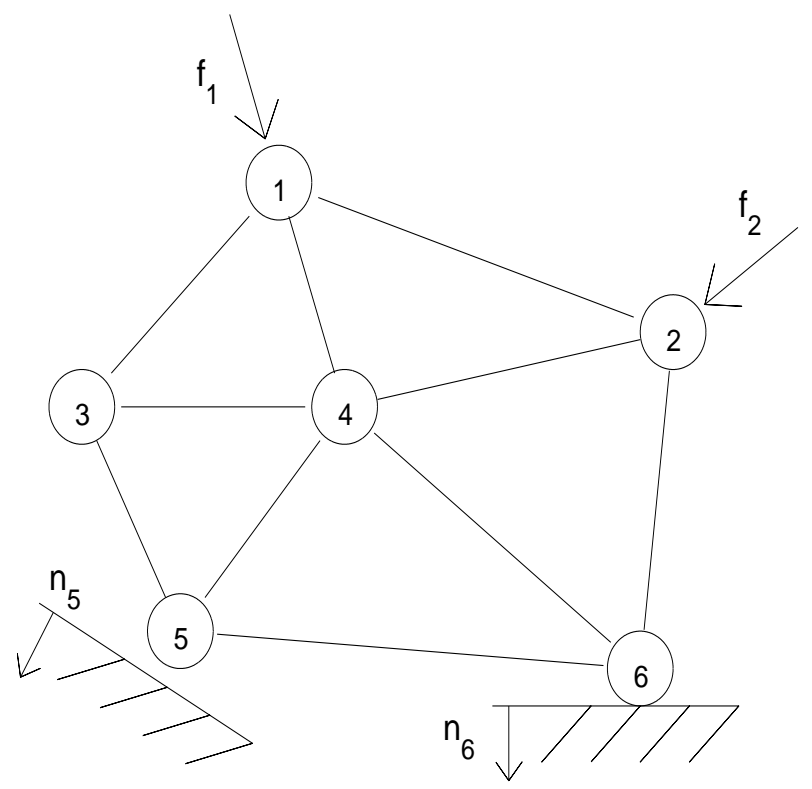

Figure 1.1: Problem setting and notation.

When $i \in I_{c}$, let $\mathbf{n}_{i}$ be a normal of unit length of the obstacle directed into the obstacle. Decompose the displacement $\overline{\mathbf{u}}_{i}$ into its normal component $\bar{u}_{i, N}=\overline{\mathbf{u}}_{i} \cdot \mathbf{n}_{i}$ and tangential component $\overline{\mathbf{u}}_{i, T}=\overline{\mathbf{u}}_{i}-\bar{u}_{i, N} \mathbf{n}_{i}$. Let us also decompose the contact force $\mathbf{r}_{i}$ into components. The normal component is $r_{i, N}=\mathbf{r}_{i} \cdot \mathbf{n}_{i}$, and the tangential component is $\mathbf{r}_{i, T}=\mathbf{r}_{i}-r_{i, N} \mathbf{n}_{i}$. This decomposition enable us to express the impenetrability of the body and obstacle according to Signorini. The contact law of Signorini reads

$$
\bar{u}_{i, N} \leq g_{i}, \quad r_{i, N} \leq 0, \quad r_{i, N}\left(\bar{u}_{i, N}-g_{i}\right)=0 \text { for all } i \in I_{c}
$$

where $g_{i}$ is the orthogonal distance from the equilibrium position of node $i$ to the obstacle. To simplify, let $\mathbf{u}_{i}=\left(\bar{u}_{i, N}-g_{i}\right) \mathbf{n}_{i}+\overline{\mathbf{u}}_{i, T}$. Then the normal component of $\mathbf{u}_{i}$ is $u_{i, N}=\bar{u}_{i, N}-g_{i}$ and the tangential component is $\mathbf{u}_{i, T}=\overline{\mathbf{u}}_{i, T}$. Hence, in terms of the displacement $\mathbf{u},(1.2)$ can be written as

$$
u_{i, N} \leq 0, \quad r_{i, N} \leq 0, \quad r_{i, N} u_{i, N}=0 \text { for all } i \in I_{c} .
$$

Equation (1.1) yields

$$
\mathbf{K u}=\mathbf{K g}+\mathbf{f}^{a p p}+\mathbf{r},
$$

where $\mathbf{g}$ is a block vector defined by $\mathbf{g}_{i, N}=g_{i} \mathbf{n}_{i}$ and $\mathbf{g}_{i, T}=\mathbf{0}$ when $i \in I_{c}$ and $\mathbf{g}_{i}=\mathbf{0}$ when $i \notin I_{c}$. Let $\mathbf{f}=\mathbf{K g}+\mathbf{f}^{a p p}$, then (1.3) is equivalent to

$$
\mathbf{K u}=\mathbf{f}+\mathbf{r} .
$$


The Coulomb law of friction defines the tangential reaction force $\mathbf{r}_{i, T}$ caused by a change of equilibrium, due to a change in $\mathbf{f}$. If $\mathbf{u}^{0}$ and $\mathbf{u}^{1}$ are two successive displacement fields, then the incremental Coulomb law of friction requires

$$
\begin{cases}\left|\mathbf{r}_{i, T}\right| \leq-\mu_{i} r_{i, N} & \\ \mathbf{u}_{i, T}^{1}-\mathbf{u}_{i, T}^{0}=\mathbf{0} & \text { if }\left|\mathbf{r}_{i, T}\right|<-\mu_{i} r_{i, N} \\ \mathbf{u}_{i, T}^{1}-\mathbf{u}_{i, T}^{0}=-\lambda_{i} \mathbf{r}_{i, T} & \text { if }\left|\mathbf{r}_{i, T}\right|=-\mu_{i} r_{i, N}>0\end{cases}
$$

where $\lambda_{i} \geq 0$ and $i \in I_{c}$. The coefficient of friction $\mu_{i}$ is assumed to be positive, $\mu_{i} \geq \mu_{\min }>0$ for all $i \in I_{c}$, where $\mu_{\text {min }}$ is a constant. The norm $|\cdot|$ is the usual Euclidean norm of a vector.

We are now in position to formulate the discrete friction problem studied in this thesis. Given an initial equilibrium $\left(\mathbf{u}^{\text {ref }}, \mathbf{r}^{\text {ref }}\right)$ as a response of a force $\mathbf{f}^{\text {ref }}$, find a new equilibrium $(\mathbf{u}, \mathbf{r})$ corresponding to the force $\mathbf{f}$ such that Coulomb's friction law and Signorini's contact law are fulfilled. Thus we assume that $\left(\mathbf{u}^{\text {ref }}, \mathbf{r}^{\text {ref }}\right)$ satisfies force equilibrium and Signorini's contact law

$$
\begin{cases}\mathbf{K} \mathbf{u}^{\mathrm{ref}}=\mathbf{f}^{\mathrm{ref}}+\mathbf{r}^{\mathrm{ref}} & \\ \mathbf{r}_{i}^{\mathrm{ref}}=\mathbf{0} & \text { when } i \notin I_{c} \\ u_{i, N}^{\mathrm{ref}} \leq 0, r_{i, N}^{\mathrm{ref}} \leq 0, u_{i, N}^{\mathrm{ref}} r_{i, N}^{\mathrm{ref}}=0 & \text { when } i \in I_{c} \\ \left|\mathbf{r}_{i, T}^{\mathrm{ref}}\right| \leq-\mu_{i} r_{i, N}^{\mathrm{ref}} & \end{cases}
$$

and find $(\mathbf{u}, \mathbf{r})$ such that

$$
\begin{cases}\mathbf{K u}=\mathbf{f}+\mathbf{r} & \\ \mathbf{r}_{i}=\mathbf{0} & \text { when } i \notin I_{c} \\ u_{i, N} \leq 0, r_{i, N} \leq 0, u_{i, N} r_{i, N}=0 & \text { when } i \in I_{c} \\ \left|\mathbf{r}_{i, T}\right| \leq-\mu_{i} r_{i, N} & \\ \mathbf{u}_{i, T}-\mathbf{u}_{i, T}^{\text {ref }}=0 & \text { if }\left|\mathbf{r}_{i, T}\right|<-\mu_{i} r_{i, N} \\ \mathbf{u}_{i, T}-\mathbf{u}_{i, T}^{\text {ref }}=-\lambda_{i} \mathbf{r}_{i, T} & \text { if }\left|\mathbf{r}_{i, T}\right|=-\mu_{i} r_{i, N}>0\end{cases}
$$

where $\lambda_{i} \geq 0$.

Introduce the inner product

$$
\langle\mathbf{f}, \mathbf{v}\rangle=\sum_{i=1}^{n} \mathbf{f}_{i} \cdot \mathbf{v}_{i}
$$

of block vectors representing force $\mathbf{f}$ and displacement $\mathbf{v}$ and let us define a norm

$$
|\mathbf{v}|=\sqrt{\sum_{i=1}^{n}\left|\mathbf{v}_{i}\right|^{2}}
$$


for displacement block vectors $\mathbf{v}$ and a corresponding norm

$$
|\mathbf{f}|=\sup _{|\mathbf{v}|=1}|\langle\mathbf{f}, \mathbf{v}\rangle|=\sqrt{\sum_{i=1}^{n}\left|\mathbf{f}_{i}\right|^{2}}
$$

for block vectors of forces. Thus, the same notation, $|\cdot|$, will be used for the Euclidean norm of forces, displacements and for the norm of block vectors of forces and displacements.

Like [11], we can now formulate (1.5) as a variational inequality. This is the content of the next lemma.

1.1. Lemma. Assume $\mathbf{u} \in \mathbb{V}=\left\{\mathbf{u}: u_{i, N} \leq 0\right.$ when $\left.i \in I_{c}\right\}$ fulfills

$$
\begin{aligned}
g(\mathbf{u}, \mathbf{v})= & \langle\mathbf{r}, \mathbf{v}-\mathbf{u}\rangle \\
& +\sum_{i \in I_{c}} \mu_{i}\left|r_{i, N}\right|\left(\left|\mathbf{v}_{i, T}-\mathbf{u}_{i, T}^{\mathrm{ref}}\right|-\left|\mathbf{u}_{i, T}-\mathbf{u}_{i, T}^{\mathrm{ref}}\right|\right) \geq 0
\end{aligned}
$$

for all $\mathbf{v} \in \mathbb{V}$, where $\mathbf{r}=\mathbf{K u}-\mathbf{f}$. Then

$$
\begin{cases}\mathbf{K u}=\mathbf{f}+\mathbf{r} & \\ \mathbf{r}_{i}=\mathbf{0} & \text { when } i \notin I_{c} \\ u_{i, N} \leq 0, r_{i, N} \leq 0, u_{i, N} r_{i, N}=0 & \text { when } i \in I_{c} \\ \left|\mathbf{r}_{i, T}\right| \leq-\mu_{i} r_{i, N} & \\ \mathbf{u}_{i, T}-\mathbf{u}_{i, T}^{\mathrm{ref}}=0 & \text { if }\left|\mathbf{r}_{i, T}\right|<-\mu_{i} r_{i, N} \\ \mathbf{u}_{i, T}-\mathbf{u}_{i, T}^{\mathrm{ref}}=-\lambda_{i} \mathbf{r}_{i, T} & \text { if }\left|\mathbf{r}_{i, T}\right|=-\mu_{i} r_{i, N}>0\end{cases}
$$

that is, $\mathbf{u}$ is a solution of (1.5) if (1.7) is satisfied.

Proof. The equilibrium of force, $\mathbf{K u}=\mathbf{f}+\mathbf{r}$, is fulfilled by definition of $\mathbf{r}$.

Let us first choose $\mathbf{v}$ in the variational inequality (1.7) so that $\mathbf{v}_{k, T}=$ $\mathbf{u}_{k, T}$ for all $k \in I_{C}$ and $v_{k, N}=u_{k, N}$ for all $k \in I_{C}$ except for $k=i$. We find

$$
r_{i, N}\left(v_{i, N}-u_{i, N}\right) \geq 0
$$

where $v_{i, N} \leq 0$ for all $i \in I_{c}$. Since $v_{i, N}$ in (1.8) is arbitrary when $i \notin I_{c}$, we have $r_{i, N}=0$ in that case. If $i \in I_{c}$ we conclude $r_{i, N} u_{i, N}=0$ and also $r_{i, N} \leq 0$.

Let $v_{k, N}=u_{k, N}$ for all $k \in I_{C}$, we find from the variational inequality (1.7) that

$$
\mathbf{r}_{i, T} \cdot\left(\mathbf{v}_{i, T}-\mathbf{u}_{i, T}\right)+\mu_{i}\left|r_{i, N}\right|\left(\left|\mathbf{v}_{i, T}-\mathbf{u}_{i, T}^{\mathrm{ref}}\right|-\left|\mathbf{u}_{i, T}-\mathbf{u}_{i, T}^{\mathrm{ref}}\right|\right) \geq 0
$$

for all $\mathbf{v}_{i, T}$ and $i \in I_{c}$. The inequality (1.9) can only hold for all $\mathbf{v}_{i, T}$ if

$$
\mathbf{r}_{i, T} \cdot \mathbf{v}_{i, T}+\mu_{i}\left|r_{i, N}\right|\left|\mathbf{v}_{i, T}\right| \geq 0
$$


for all $\mathbf{v}_{i, T}$ and $i \in I_{c}$, which yields $\left|\mathbf{r}_{i, T}\right| \leq \mu_{i}\left|r_{i, N}\right|=-\mu_{i} r_{i, N}$. Now, let $\mathbf{v}_{i, T}=\mathbf{u}_{i, T}^{\text {ref }}$ in (1.9), then

$$
\mathbf{r}_{i, T} \cdot\left(\mathbf{u}_{i, T}^{\mathrm{ref}}-\mathbf{u}_{i, T}\right)-\mu_{i}\left|r_{i, N}\right|\left(\left|\mathbf{u}_{i, T}-\mathbf{u}_{i, T}^{\mathrm{ref}}\right|\right) \geq 0
$$

so that

$$
\begin{cases}\mathbf{u}_{i, T}-\mathbf{u}_{i, T}^{\mathrm{ref}}=0 & \text { if }\left|\mathbf{r}_{i, T}\right|<-\mu_{i} r_{i, N} \\ \mathbf{u}_{i, T}-\mathbf{u}_{i, T}^{\mathrm{ref}}=-\lambda_{i} \mathbf{r}_{i, T} & \text { if }\left|\mathbf{r}_{i, T}\right|=-\mu_{i} r_{i, N}>0\end{cases}
$$

where $\lambda_{i} \geq 0$. 


\section{Chapter 2}

\section{Existence results for discrete contact problems}

In this chapter we examine the variational inequality (1.7) to find compatibility conditions on the applied force that ensure the existence of solutions to the friction problem (1.5).

\section{Existence of solutions in the coercive case}

Let us to prove the existence of a solution to the friction problem (1.5) or (1.7) when the stiffness matrix $\mathbf{K}$ is positive definite. The positive definiteness requires

$$
\langle\mathbf{K u}, \mathbf{u}\rangle \geq \lambda_{\min }|\mathbf{u}|^{2}
$$

where $\lambda_{\min }>0$ is the smallest eigenvalue of $\mathbf{K}$. We have the following theorem.

2.1. Theorem. If the stiffness matrix $\mathbf{K}$ is positive definite, that is (2.1) holds, then the friction problem (1.5) has at least one solution.

Proof. We use a fixed point theorem, which in a more general form originates from Brezis et.al. [5] and which generalizes results from Fan [13] (see also [30]):

Let $\mathbb{M}$ be a convex subset of $\mathbb{R}^{m}$ and let $\ell(x, y)$ be a real valued function defined on $\mathbb{M} \times \mathbb{M}$ such that

I. For every fixed $y \in \mathbb{M}, \ell(x, y)$ is a continuous function of $x \in \mathbb{M}$.

II. There is a compact set $L \subset R^{m}$ and an element $y_{0} \in L \cap C$ such that $\ell\left(x, y_{0}\right)<0$ for all $x \in \mathbb{M}$ such that $x \notin L$. 
III. For every fixed $x \in \mathbb{M}$, the set $\{y \in \mathbb{M}: \ell(x, y)<0\}$ is convex.

IV. $\ell(x, x) \geq 0$ for all $x \in \mathbb{M}$.

Then there exists $x_{0} \in L \cap \mathbb{M}$ such that

$$
\ell\left(x_{0}, y\right) \geq 0 \text { for all } y \in \mathbb{M} \text {. }
$$

It suffices to verify the conditions (I), (II), (III) and (IV) of the fixed point theorem to guarantee the existence of a solution of (1.7). Let $\mathbb{M}=$ $\mathbb{V}=\left\{\mathbf{u}: u_{i, N} \leq 0\right.$ when $\left.i \in I_{c}\right\}$ and $\ell=g$ from (1.7), then condition (I) is satisfied since $g(\mathbf{u}, \mathbf{v})$ is continuous with respect to $\mathbf{u}$ for a fixed $\mathbf{v}$. To verify (II), we prove that

$$
L=\left\{\mathbf{u}: g\left(\mathbf{u}, \mathbf{u}^{\text {ref }}\right) \geq 0\right\}
$$

is compact and notice that $\mathbf{u}^{\text {ref }} \in L \cap \mathbb{V}$. It suffices to show that $L$ is bounded, since closedness follows from the continuity of $g\left(\mathbf{u}, \mathbf{u}^{\text {ref }}\right)$. Let us consider a sequence $\mathbf{v}^{k} \in \mathbb{V}$ such that $\left|\mathbf{v}^{k}\right| \rightarrow \infty$, and use $\mathbf{r}^{k}=\mathbf{K} \mathbf{v}^{k}-\mathbf{f}$ to find

$$
\begin{aligned}
\left\langle\mathbf{r}^{k}, \mathbf{u}^{\text {ref }}-\mathbf{v}^{k}\right\rangle= & \left\langle\mathbf{K} \mathbf{v}^{k}-\mathbf{f}, \mathbf{u}^{\text {ref }}-\mathbf{v}^{k}\right\rangle \\
= & -\left\langle\mathbf{K} \mathbf{v}^{k}, \mathbf{v}^{k}\right\rangle+\left\langle\mathbf{K} \mathbf{v}^{k}, \mathbf{u}^{\text {ref }}\right\rangle+\left\langle\mathbf{f}, \mathbf{v}^{k}\right\rangle-\left\langle\mathbf{f}, \mathbf{u}^{\text {ref }}\right\rangle \\
\leq & -\lambda_{\text {min }}\left|\mathbf{v}^{k}\right|^{2}+\left(\lambda_{\max }\left|\mathbf{u}^{\text {ref }}\right|+|\mathbf{f}|\right)\left|\mathbf{v}^{k}\right|+\left\langle\mathbf{f}, \mathbf{u}^{\text {ref }}\right\rangle \\
& \rightarrow-\infty \text { when } k \rightarrow \infty
\end{aligned}
$$

where $\lambda_{\min }$ and $\lambda_{\max }$ denotes the smallest and largest eigenvalue of $\mathbf{K}$. This yields

$$
g\left(\mathbf{v}^{k}, \mathbf{u}^{\text {ref }}\right)=\left\langle\mathbf{r}^{k}, \mathbf{u}^{\text {ref }}-\mathbf{v}^{k}\right\rangle-\sum_{i \in I_{c}} \mu_{i}\left|r_{i, N}^{k}\right|\left(\left|\mathbf{v}_{i, T}^{k}-\mathbf{u}_{i, T}^{\mathrm{ref}}\right|\right) \rightarrow-\infty
$$

which contradicts $\mathbf{v}_{k} \in L$, so that $L$ is bounded, which verifies (II). Let us continue with (III). If $\mathbf{u}$ is kept fixed, then $\mathbf{r}$ is constant. From the linearity of $\langle\mathbf{r}, \mathbf{v}-\mathbf{u}\rangle$ and convexity of

$$
\sum_{i=1}^{n} \mu_{i}\left|r_{i, N}\right|\left(\left|\mathbf{v}_{i, T}-\mathbf{u}_{i, T}^{\mathrm{ref}}\right|-\left|\mathbf{u}_{i, T}-\mathbf{u}_{i, T}^{\mathrm{ref}}\right|\right)
$$

with respect to $\mathbf{v}$ for every fixed $\mathbf{u} \in \mathbb{V}$, it follows that $g(\mathbf{u}, \mathbf{v})$ is convex, and this yields the convexity of the set $\{\mathbf{v} \in \mathbb{V}: g(\mathbf{u}, \mathbf{v})<0\}$. Finally, (IV) is fulfilled since $g(\mathbf{u}, \mathbf{u})=0$ for all $\mathbf{u} \in \mathbb{V}$.

Theorem 2.1 is a discrete version of the theorems by Duvaut [9], Demkowicz et. al. [8] and Cocou [6]. It is also proved in Klarbring et. al. [25]. 


\section{Existence of solutions in the noncoercive case}

If the truss considered in the friction problem is insufficiently anchored, then the stiffness matrix $\mathbf{K}$ is not positive definite, so (2.1) is not fulfilled. However, $\mathbf{K}$ is still semidefinite,

$$
\langle\mathbf{K u}, \mathbf{u}\rangle \geq 0
$$

for all displacements $\mathbf{u}$. Let us call elements of the set $\mathcal{N}=\{\mathbf{u}: \mathbf{K u}=\mathbf{0}\}$ rigid body displacements. We will consider the case when $\mathcal{N} \neq\{\mathbf{0}\}$, and call such friction problems semicoercive or noncoercive.

Let us introduce the auxiliary matrix $\mathbf{D}$, which is a positive definite matrix so that $d_{\text {max }}|\mathbf{v}|^{2} \geq\langle\mathbf{v}, \mathbf{D v}\rangle \geq d_{\text {min }}|\mathbf{v}|^{2}$ for some positive constants $d_{\min }$ and $d_{\max }$. The aim of this section is to prove compatibility conditions on the applied force so that there are solutions of the semicoercive discrete friction problem. We perturb the stiffness matrix $\mathbf{K}$ by adding $\varepsilon \mathbf{D}$, where $\varepsilon$ is a positive real number, to obtain a positive definite matrix $\mathbf{K}+\varepsilon \mathbf{D}$. Then, for every $\varepsilon_{n}$ in a sequence $\left\{\varepsilon_{n}\right\}_{n=1}^{\infty}$ where $\varepsilon_{n}>0$ for all $n$, the problem

$$
\begin{cases}\left(\mathbf{K}+\varepsilon_{n} \mathbf{D}\right) \mathbf{u}^{n}=\mathbf{f}+\mathbf{r}^{n} & \\ \mathbf{r}_{i}=\mathbf{0} & \text { when } i \notin I_{c} \\ u_{i, N}^{n} \leq 0, r_{i, N}^{n} \leq 0, u_{i, N}^{n} r_{i, N}^{n}=0 & \text { when } i \in I_{c} \\ \left|\mathbf{r}_{i, T}^{n}\right| \leq-\mu_{i} r_{i, N}^{n} & \\ \mathbf{u}_{i, T}^{n}-\mathbf{u}_{i, T}^{\text {ref }}=0 & \text { if }\left|\mathbf{r}_{i, T}^{n}\right|<-\mu_{i} r_{i, N}^{n} \\ \mathbf{u}_{i, T}^{n}-\mathbf{u}_{i, T}^{\text {ref }}=-\lambda_{i}^{n} \mathbf{r}_{i, T}^{n} & \text { if }\left|\mathbf{r}_{i, T}^{n}\right|=-\mu_{i} r_{i, N}^{n}>0\end{cases}
$$

has a solution $\left(\mathbf{u}^{n}, \mathbf{r}^{n}\right)$ according to Theorem 2.1 , where $\left(\mathbf{u}^{\text {ref }}, \mathbf{r}^{\text {ref }}\right)$ is assumed to fulfill the equilibrium and contact conditions (1.4). We shall consider the sequence of displacements and reaction forces $\left(\mathbf{u}^{n}, \mathbf{r}^{n}\right)$ obtained as solutions of $(2.2)$ when $\varepsilon_{n} \rightarrow 0^{+}$as $n \rightarrow \infty$. If one $\varepsilon_{n}$ yields several solutions, select one of these solutions arbitrarily.

Before studying (2.2), we recapture two tools from real analysis and linear algebra:

1. Bolzano-Weierstrass theorem, which states that a bounded sequence in a finite dimensional linear space has a convergent subsequence. In the following we focus on the convergent subsequences to determine the properties of the limits, and to simplify notation we often assume that the entire sequence converges, without loss of generality.

2. The stiffness matrix $\mathbf{K}$ is symmetric, hence if $\mathbf{y}_{n}$ is a bounded sequence in the range $\mathcal{R}=\{\mathbf{y}: \mathbf{y}=\mathbf{K} \mathbf{x}$ for some $\mathbf{x}\}$ of $\mathbf{K}$ then there is a bounded sequence $\mathbf{x}_{n}$ such that $\mathbf{y}_{n}=\mathbf{K} \mathbf{x}_{n}$ and $\mathbf{x}_{n} \in \mathcal{R}$.

The following lemma establishes some properties of the auxiliary friction problem (2.2). 
2.2. Lemma. I. Define $\mathbf{u}_{T}^{\mathrm{ref}}$ by $\left(\mathbf{u}_{T}^{\mathrm{ref}}\right)_{i}=\mathbf{0}$ when $i \notin I_{c}$ and

$$
\left\{\begin{array}{l}
\left(\mathbf{u}_{T}^{\mathrm{ref}}\right)_{i, N}=0 \\
\left(\mathbf{u}_{T}^{\mathrm{ref}}\right)_{i, T}=\mathbf{u}_{i, T}^{\mathrm{ref}}
\end{array}\right.
$$

when $i \in I_{c}$. Let us also define

$$
\mathbf{w}^{n}=\left\{\begin{array}{cl}
\frac{\mathbf{u}^{n}-\mathbf{u}_{T}^{\mathrm{ref}}}{\left|\mathbf{u}^{n}-\mathbf{u}_{T}^{\mathrm{ref}}\right|} & \text { if }\left|\mathbf{u}^{n}-\mathbf{u}_{T}^{\mathrm{ref}}\right|>0 \\
\mathbf{0} & \text { otherwise }
\end{array}\right.
$$

Then

$$
\mathbf{w}_{i}^{n} \cdot \mathbf{r}_{i}^{n}=-\mu_{i}\left|r_{i, N}^{n}\right|\left|\mathbf{w}_{i, T}^{n}\right|
$$

and

$$
\left\langle\mathbf{r}^{n}, \mathbf{w}^{n}\right\rangle=\sum_{i} \mathbf{w}_{i}^{n} \cdot \mathbf{r}_{i}^{n} \leq 0
$$

II. Let us assume $\left|\mathbf{u}^{n}\right| \rightarrow \infty$ when $\varepsilon_{n} \rightarrow 0$. Then there is a convergent subsequence of $\mathbf{w}^{n}$, such that $\mathbf{w}^{n} \rightarrow \mathbf{w}$, where $\mathbf{w} \in \mathcal{N}$ and $|\mathbf{w}|=1$. Moreover

$$
\lim _{n \rightarrow \infty} \varepsilon_{n} \mathbf{u}^{n}=\alpha \mathbf{w}
$$

where $\alpha \geq 0$, and

$$
\left|\varepsilon_{n} \mathbf{u}^{n}\right|<C_{1}<\infty \text { for all } n
$$

where $C_{1}$ is a constant.

III. The sequence of contact forces $\mathbf{r}^{n}$ obtained as a solution of the auxiliary problem (2.2) when $\varepsilon_{n} \rightarrow 0$ is bounded.

Proof. I. If $\left|\mathbf{u}^{n}-\mathbf{u}_{T}^{\text {ref }}\right|=0$ then $\mathbf{w}^{n}=\mathbf{0}$, so that (2.3) and (2.4) are fulfilled. If $\left|\mathbf{u}^{n}-\mathbf{u}_{T}^{\text {ref }}\right|>0$, then from (2.2) follows that for any node $i$ we have

$$
\begin{aligned}
\mathbf{w}_{i}^{n} \cdot \mathbf{r}_{i}^{n} & =\frac{1}{\left|\mathbf{u}^{n}-\mathbf{u}_{T}^{\mathrm{ref}}\right|}\left(u_{i, N}^{n} r_{i, N}^{n}+\left(\mathbf{u}_{i, T}^{n}-\mathbf{u}_{i, T}^{\mathrm{ref}}\right) \cdot \mathbf{r}_{i, T}^{n}\right) \\
& =-\mu_{i}\left|r_{i, N}^{n}\right| \frac{\left|\mathbf{u}_{i, T}^{n}-\mathbf{u}_{i, T}^{\mathrm{ref}}\right|}{\left|\mathbf{u}^{n}-\mathbf{u}_{T}^{\text {ref }}\right|}=-\mu_{i}\left|r_{i, N}^{n}\right|\left|\mathbf{w}_{i, T}^{n}\right|
\end{aligned}
$$

which yields

$$
\left\langle\mathbf{r}^{n}, \mathbf{w}^{n}\right\rangle=\sum_{i} \mathbf{w}_{i}^{n} \cdot \mathbf{r}_{i}^{n} \leq 0
$$


II. The solutions of (2.2) fulfill $\left(\mathbf{K}+\varepsilon_{n} \mathbf{D}\right) \mathbf{u}^{n}=\mathbf{f}+\mathbf{r}^{n}$, so that

$$
-\mathbf{r}^{n}+\varepsilon_{n} \mathbf{D}\left(\mathbf{u}^{n}-\mathbf{u}_{T}^{\mathrm{ref}}\right)+\mathbf{K}\left(\mathbf{u}^{n}-\mathbf{u}_{T}^{\mathrm{ref}}\right)=\mathbf{f}-\mathbf{K} \mathbf{u}_{T}^{\mathrm{ref}}-\varepsilon_{n} \mathbf{D} \mathbf{u}_{T}^{\mathrm{ref}}
$$

and hence multiplying by $\mathbf{w}^{n}$

$$
\begin{aligned}
0 \leq & -\left\langle\mathbf{r}^{n}, \mathbf{w}^{n}\right\rangle+\varepsilon_{n}\left|\mathbf{u}^{n}-\mathbf{u}_{T}^{\text {ref }}\right|\left\langle\mathbf{w}^{n}, \mathbf{D} \mathbf{w}^{n}\right\rangle \\
& +\left|\mathbf{u}^{n}-\mathbf{u}_{T}^{\text {ref }}\right|\left\langle\mathbf{w}^{n}, \mathbf{K} \mathbf{w}^{n}\right\rangle \\
= & \left\langle\mathbf{f}, \mathbf{w}^{n}\right\rangle-\left\langle\mathbf{K} \mathbf{u}_{T}^{\text {ref }}, \mathbf{w}^{n}\right\rangle-\varepsilon_{n}\left\langle\mathbf{D} \mathbf{u}_{T}^{\text {ref }}, \mathbf{w}^{n}\right\rangle \\
\leq & |\mathbf{f}|+\left|\mathbf{K} \mathbf{u}_{T}^{\text {ref }}\right|+\varepsilon_{n}\left|\mathbf{D} \mathbf{u}_{T}^{\text {ref }}\right| \leq C_{2}<\infty
\end{aligned}
$$

where $C_{2}$ is a constant.

Since $\left|\mathbf{w}^{n}\right|=1$, there is a convergent subsequence of $\left\{\mathbf{w}^{n}\right\}_{n=1}^{\infty}$. For simplicity, assume that $\mathbf{w}^{n} \rightarrow \mathbf{w}$. From (2.6) follows that $\left\langle\mathbf{K} \mathbf{w}^{n}, \mathbf{w}^{n}\right\rangle \rightarrow$ 0 when $n \rightarrow \infty$, so that $\langle\mathbf{w}, \mathbf{K w}\rangle=0$. Since $\mathbf{K}$ is positive semidefinite, this implies $\mathbf{K w}=\mathbf{0}$, and thus $\mathbf{w} \in \mathcal{N}$.

Equation (2.6) yields

$$
\varepsilon_{n}\left|\mathbf{u}^{n}-\mathbf{u}_{T}^{\mathrm{ref}}\right| \leq C_{2} /\left\langle\mathbf{w}^{n}, \mathbf{D} \mathbf{w}^{n}\right\rangle \leq C_{2} / d_{\min }<\infty,
$$

and hence we can assume that the sequence $\left\{\varepsilon_{n}\left|\mathbf{u}^{n}-\mathbf{u}_{T}^{\text {ref }}\right|\right\}_{n=1}^{\infty}$ converges, $\varepsilon_{n}\left|\mathbf{u}^{n}-\mathbf{u}^{\text {ref }}\right| \rightarrow \alpha<\infty$ when $n \rightarrow \infty$. Thus

$$
\lim _{n \rightarrow \infty} \varepsilon_{n} \mathbf{u}^{n}=\lim _{n \rightarrow \infty} \varepsilon_{n}\left|\mathbf{u}^{n}-\mathbf{u}_{T}^{\mathrm{ref}}\right| \frac{\mathbf{u}^{n}-\mathbf{u}_{T}^{\mathrm{ref}}}{\left|\mathbf{u}^{n}-\mathbf{u}_{T}^{\mathrm{ref}}\right|}=\alpha \mathbf{w} .
$$

and also $\left|\varepsilon_{n} \mathbf{u}^{n}\right|<C_{1}<\infty$ for all $n$, where $C_{1}$ is a constant.

III. To prove this proposition, let us assume $\left|\mathbf{r}^{n}\right| \rightarrow \infty$ to obtain a contradiction. Since $\mathbf{r}^{n}=\varepsilon_{n} \mathbf{D} \mathbf{u}^{n}+\mathbf{K} \mathbf{u}^{n}-\mathbf{f}$ is bounded if $\mathbf{u}^{n}$ is bounded, we also have $\left|\mathbf{u}^{n}\right| \rightarrow \infty$. Let $\mathcal{F}^{n}=\mathbf{f}-\varepsilon_{n} \mathbf{D} \mathbf{u}^{n}$, then

$$
\left|\mathcal{F}^{n}\right|=\left|\mathbf{f}-\varepsilon_{n} \mathbf{D} \mathbf{u}^{n}\right| \leq C_{1} d_{\max }+|\mathbf{f}| \leq C_{3}<\infty
$$

where (2.5) was used and $C_{3}$ is a positive constant.

Let us define $\mathbf{R}^{n}$ by $\mathbf{R}^{n}=\mathbf{K} \mathbf{u}^{n}=\mathbf{r}^{n}+\mathbf{f}-\varepsilon_{n} \mathbf{D} \mathbf{u}^{n}=\mathbf{r}^{n}+\mathcal{F}^{n}$, then it follows from (2.7) that $\left|\mathbf{R}^{n}\right| \geq\left|\mathbf{r}^{n}\right|-\left|\mathcal{F}^{n}\right| \rightarrow \infty$ so we can assume $\left|\mathbf{R}^{n}\right|>0$ for all $n$. Let $\mathbf{x}^{n}=\mathbf{R}^{n} /\left|\mathbf{R}^{n}\right|$, then $\mathbf{r}^{n}=\left|\mathbf{R}^{n}\right| \mathbf{x}^{n}-\mathcal{F}^{n}$.

We have $\left|\mathbf{x}^{n}\right|=1$ and $\mathbf{x}^{n} \in \mathcal{R}$, so that we can assume convergence, $\mathbf{x}^{n} \rightarrow \mathbf{x} \in \mathcal{R}$, where $\mathcal{R}$ is the range of $\mathbf{K}$. If $\left|\mathbf{x}_{i}\right| \neq 0$, then

$$
\lim _{n \rightarrow \infty} \frac{\mathbf{r}_{i}^{n}}{\left|\mathbf{r}_{i}^{n}\right|}=\sigma_{i} \mathbf{x}_{i}
$$

where $\sigma_{i} \geq 1$. Let $\sigma=\max _{i} \sigma_{i}$. Using (2.3) and the inequality

$$
\left|\mathbf{r}_{i}^{n}\right| \leq\left|r_{i, N}^{n}\right|+\left|\mathbf{r}_{i, T}^{n}\right| \leq\left(1+\mu_{i}\right)\left|r_{i, N}^{n}\right| \leq\left(1+\max _{i \in I c} \mu_{i}\right)\left|r_{i, N}^{n}\right|
$$


and the observation $\mathbf{u}_{i}=\mathbf{u}_{i, T}$ if $\mathbf{r}_{i}^{n} \neq 0$, we conclude

$$
\begin{aligned}
\mathbf{r}_{i}^{n} \cdot\left(\mathbf{u}_{i}^{n}-\mathbf{u}_{i, T}^{\mathrm{ref}}\right) & =-\mu_{i}\left|r_{i, N}^{n}\right|\left|\mathbf{u}_{i, T}^{n}-\mathbf{u}_{i, T}^{\mathrm{ref}}\right| \\
& \leq-\frac{\mu_{\min }}{1+\max _{i \in I c} \mu_{i}}\left|\mathbf{r}_{i}^{n}\right|\left|\mathbf{u}_{i}^{n}-\mathbf{u}_{i, T}^{\mathrm{ref}}\right| \leq 0
\end{aligned}
$$

and thus

$$
\mathbf{x}_{i} \cdot\left(\mathbf{u}_{i}^{n}-\mathbf{u}_{i, T}^{\mathrm{ref}}\right) \leq-\frac{\mu_{\min }}{2 \sigma\left(1+\max _{i \in I c} \mu_{i}\right)}\left|\mathbf{u}_{i}^{n}-\mathbf{u}_{i, T}^{\mathrm{ref}}\right| \leq 0
$$

for large $n$, which implies

$$
\left\langle\mathbf{x}, \mathbf{u}^{n}-\mathbf{u}_{T}^{\mathrm{ref}}\right\rangle=\sum_{i} \mathbf{x}_{i} \cdot\left(\mathbf{u}_{i}^{n}-\mathbf{u}_{i}^{\mathrm{ref}}\right) \leq 0 .
$$

But since $\mathbf{x}^{n} \in \mathcal{R}$ and $\left|\mathbf{x}^{n}\right|$ is a bounded sequence, there is a bounded sequence $\mathbf{y}^{n} \in \mathcal{R}$ such that $\mathbf{x}^{n}=\mathbf{K} \mathbf{y}^{n}$. Assume $\mathbf{y}^{n} \rightarrow \mathbf{y}$, then $\mathbf{y} \neq \mathbf{0}$ since $\mathbf{K y}=\mathbf{x} \neq \mathbf{0}$. We obtain

$$
\mathbf{K}\left(\mathbf{u}^{n}-\left|\mathbf{R}^{n}\right| \mathbf{y}^{n}\right)=\mathbf{R}^{n}-\mathbf{R}^{n}=0 .
$$

Hence, let us define $\mathbf{z}^{n}=\mathbf{u}^{n}-\left|\mathbf{R}^{n}\right| \mathbf{y}^{n} \in \mathcal{N}$. Note that $\mathbf{u}_{T}^{\text {ref }}$ is bounded, $\left\langle\mathbf{x}, \mathbf{z}^{n}\right\rangle=0$ and $\lim _{n \rightarrow \infty}\left\langle\mathbf{x}, \mathbf{y}^{n}\right\rangle=\langle\mathbf{K y}, \mathbf{y}\rangle>0$. We find

$$
\begin{aligned}
\left\langle\mathbf{x}, \mathbf{u}^{n}-\mathbf{u}_{T}^{\mathrm{ref}}\right\rangle & =\left\langle\mathbf{x}, \mathbf{z}^{n}+\left|\mathbf{R}^{n}\right| \mathbf{y}^{n}-\mathbf{u}_{T}^{\mathrm{ref}}\right\rangle \\
& =\left\langle\mathbf{x}, \mathbf{z}^{n}\right\rangle+\left|\mathbf{R}^{n}\right|\left\langle\mathbf{x}, \mathbf{y}^{n}\right\rangle-\left\langle\mathbf{x}, \mathbf{u}_{T}^{\mathrm{ref}}\right\rangle \rightarrow \infty
\end{aligned}
$$

which contradicts (2.9). Thus $\left|\mathbf{r}^{n}\right|$ is a bounded sequence, and we can assume $\mathbf{r}^{n} \rightarrow \mathbf{r}$.

2.3. Theorem. Let $\mathbf{D}$ be an arbitrary positive definite matrix. Then lack of solutions to the discrete incremental noncoercive friction problem (1.5) implies that for any choice of the positive definite matrix $\mathbf{D}$, there exist a rigid body velocity $\mathbf{w} \in \mathcal{N}$ such that $\mathbf{w} \neq \mathbf{0}$, a contact force field $\mathbf{r}$ and $\alpha \geq 0$ that fulfill

$$
\alpha\langle\mathbf{D w}, \mathbf{v}\rangle=\left\langle\mathbf{f}^{\text {app }}, \mathbf{v}\right\rangle+\langle\mathbf{r}, \mathbf{v}\rangle
$$

for all $\mathbf{v} \in \mathcal{N}=\{\mathbf{v}: \mathbf{K} \mathbf{v}=\mathbf{0}\}$, where $\mathbf{w} \in \mathbb{V}=\left\{\mathbf{u}: u_{i, N} \leq 0\right.$ when $i \in$ $\left.I_{c}\right\}$. The contact forces $\mathbf{r}_{i}$ fulfill $\left|\mathbf{r}_{i, T}\right| \leq-\mu_{i} r_{i, N}$ and correspond to $\mathbf{w}$ by $w_{i, N} r_{i, N}=0$ and

$$
\begin{cases}\mathbf{w}_{i, T}=0 & \text { if }\left|\mathbf{r}_{i, T}\right|<-\mu_{i} r_{i, N} \\ \mathbf{w}_{i, T}=-\lambda_{i} \mathbf{r}_{i, T} & \text { if }\left|\mathbf{r}_{i, T}\right|=-\mu_{i} r_{i, N}>0\end{cases}
$$

where $\lambda_{i} \geq 0$. 
Proof. Let us consider the sequence of solutions $\left\{\mathbf{u}^{n}\right\}_{n=1}^{\infty}$ to the auxiliary problem (2.2) as $\varepsilon_{n} \rightarrow 0$. If $\left|\mathbf{u}^{n}\right|$ is a bounded sequence, then there is a convergent subsequence, $\mathbf{u}^{n} \rightarrow \mathbf{u}$ and $\mathbf{r}^{n} \rightarrow \mathbf{r}$ as $n \rightarrow \infty$, according to Lemma 2.2 (III). If we let $n \rightarrow \infty$ in (2.2) then we obtain a solution of the semicoercive friction problem (1.5). Also, note that

$$
\langle\mathbf{f}, \mathbf{v}\rangle=\left\langle\mathbf{K g}+\mathbf{f}^{\text {app }}, \mathbf{v}\right\rangle=\left\langle\mathbf{f}^{\text {app }}, \mathbf{v}\right\rangle,
$$

where $\mathbf{f}^{\text {app }}$ is the applied force defined in (1.3).

If $\left|\mathbf{u}^{n}\right|$ is not bounded, then without loss of generality we can assume $\left|\mathbf{u}^{n}\right| \rightarrow \infty$ and $\mathbf{r}^{n} \rightarrow \mathbf{r}$ as $n \rightarrow \infty$. From 2.2 (II) we obtain

$$
\begin{aligned}
0= & \left\langle\mathbf{K} \mathbf{u}^{n}, \mathbf{v}\right\rangle \\
= & \langle\mathbf{f}, \mathbf{v}\rangle+\left\langle\mathbf{r}^{n}, \mathbf{v}\right\rangle-\varepsilon_{n}\left\langle\mathbf{D} \mathbf{u}_{n}, \mathbf{v}\right\rangle \\
& \rightarrow\langle\mathbf{f}, \mathbf{v}\rangle+\langle\mathbf{r}, \mathbf{v}\rangle-\alpha\langle\mathbf{D w}, \mathbf{v}\rangle
\end{aligned}
$$

for all $\mathbf{v} \in \mathcal{N}$ where $\mathbf{w} \in \mathcal{N}$ and $\alpha \geq 0$. We obtain (2.10) from (2.12).

Also, from (2.2) follows

$$
\begin{cases}\mathbf{r}_{i}^{n}=\mathbf{0} & \text { when } i \notin I_{c} \\ w_{i, N}^{n} \leq 0, r_{i, N}^{n} \leq 0, w_{i, N}^{n} \cdot r_{i, N}^{n}=0 & \text { when } i \in I_{c} \\ \left|\mathbf{r}_{i, T}^{n}\right| \leq-\mu_{i} r_{i, N}^{n} & \\ \mathbf{w}_{i, T}^{n}=0 & \text { if }\left|\mathbf{r}_{i, T}^{n}\right|<-\mu_{i} r_{i, N}^{n} \\ \mathbf{w}_{i, T}^{n}=-\frac{\lambda_{i}^{n}}{\mid \mathbf{u}^{n}-\mathbf{u}^{\text {ref } \mid}} \mathbf{r}_{i, T}^{n} & \text { if }\left|\mathbf{r}_{i, T}^{n}\right|=-\mu_{i} r_{i, N}^{n}>0 .\end{cases}
$$

Hence if $n \rightarrow \infty$, we obtain

$$
\begin{cases}\mathbf{r}_{i}=\mathbf{0} & \text { when } i \notin I_{c} \\ w_{i, N} \leq 0, r_{i, N} \leq 0, w_{i, N} \cdot r_{i, N}=0 & \text { when } i \in I_{c} \\ \left|\mathbf{r}_{i, T}\right| \leq-\mu_{i} r_{i, N} & \\ \mathbf{w}_{i, T}=0 & \text { if }\left|\mathbf{r}_{i, T}\right|<-\mu_{i} r_{i, N} \\ \mathbf{w}_{i, T}=-\lambda_{i} \mathbf{r}_{i, T} & \text { if }\left|\mathbf{r}_{i, T}\right|=-\mu_{i} r_{i, N}>0\end{cases}
$$

where $\lambda_{i}=\frac{\left|\mathbf{w}_{i, T}\right|}{\left|\mathbf{r}_{i, T}\right|}$.

\section{Interpretation}

Assume that the applied force is such that the incremental friction problem (1.5) lacks solutions. Let us consider a rigid truss exposed to the same applied force. We will show that the dynamical problem for this rigid truss has a solution where the truss is in motion, which indicates that the quasistatic modelling of the truss is inadequate in this case.

By dynamical solution we mean the classical solution obtained from Newton's second law of motion. To use this equation we have to verify 
that no collisions with the obstacle will occur, so that a smooth solution is possible.

To find the motion of a rigid truss we use equations of linear and angular momentum (see [16]). If we assign the mass $m_{i}$ to each node, the equations read

$$
\begin{gathered}
\sum_{i=1}^{n} m_{i} \frac{\mathrm{d}^{2} \mathbf{q}_{i}}{\mathrm{~d} t^{2}}=\sum_{i=1}^{n} \mathbf{f}_{i}^{\mathrm{app}}+\mathbf{r}_{i} \\
\sum_{i=1}^{n} m_{i}\left(\mathbf{q}_{i} \times \frac{\mathrm{d}^{2} \mathbf{q}_{i}}{\mathrm{~d} t^{2}}\right)=\sum_{i=1}^{n}\left(\mathbf{q}_{i} \times \mathbf{f}_{i}^{\mathrm{app}}+\mathbf{q}_{i} \times \mathbf{r}_{i}\right)
\end{gathered}
$$

where $\mathbf{q}_{i}$ is the radius vector of node $i$ from some given origin and $\times$ denotes the cross product. Let $\mathbf{M}$ be a diagonal matrix with the masses $m_{i}$ as diagonal elements. The rigid body motions $\mathcal{N}$ consist of translations and rotations, let us consider two vectors $\mathbf{e}^{x}$ and $\mathbf{e}^{x x}$ in $\mathcal{N}$ that are defined by

$$
\mathbf{e}_{i}^{x}=\hat{\mathbf{x}}, \quad \mathbf{e}_{i}^{x x}=\hat{\mathbf{x}} \times \mathbf{q}_{i}
$$

where $\hat{\mathbf{x}}$ is a unit vector in the $\mathbf{x}$-direction. We find from (2.13)

$$
\left\langle\mathbf{e}^{x}, \mathbf{M} \frac{\mathrm{d}^{2} \mathbf{u}(t)}{\mathrm{d} t^{2}}\right\rangle=\sum_{i=1}^{n} m_{i} \hat{\mathbf{x}} \cdot \frac{\mathrm{d}^{2} \mathbf{q}_{i}}{\mathrm{~d} t^{2}}=\sum_{i=1}^{n} \hat{\mathbf{x}} \cdot\left(\mathbf{f}_{i}^{\text {app }}+\mathbf{r}_{i}\right)=\left\langle\mathbf{e}^{x}, \mathbf{f}^{\text {app }}+\mathbf{r}\right\rangle
$$

and

$$
\begin{aligned}
\left\langle\mathbf{e}^{x x}, \mathbf{M} \frac{\mathrm{d}^{2} \mathbf{u}(t)}{\mathrm{d} t^{2}}\right\rangle & =\sum_{i=1}^{n} m_{i}\left(\hat{\mathbf{x}} \times \mathbf{q}_{i}\right) \cdot \frac{\mathrm{d}^{2} \mathbf{q}_{i}}{\mathrm{~d} t^{2}}=\sum_{i=1}^{n} m_{i} \hat{\mathbf{x}} \cdot\left(\mathbf{q}_{i} \times \frac{\mathrm{d}^{2} \mathbf{q}_{i}}{\mathrm{~d} t^{2}}\right) \\
& =\sum_{i=1}^{n} \hat{\mathbf{x}} \cdot\left(\mathbf{q}_{i} \times \mathbf{f}_{i}^{\mathrm{app}}+\mathbf{q}_{i} \times \mathbf{r}_{i}\right) \\
& =\sum_{i=1}^{n}\left(\hat{\mathbf{x}} \times \mathbf{q}_{i}\right) \cdot\left(\mathbf{f}_{i}^{\text {app }}+\mathbf{r}_{i}\right)=\left\langle\mathbf{e}^{x x}, \mathbf{f}^{\text {app }}+\mathbf{r}\right\rangle
\end{aligned}
$$

Since we can compose a basis for $\mathcal{N}$ of vectors representing rotations and translations we conclude

$$
\left\langle\mathbf{v}, \mathbf{M} \frac{\mathrm{d}^{2} \mathbf{u}(t)}{\mathrm{d} t^{2}}\right\rangle=\left\langle\mathbf{v}, \mathbf{f}^{\text {app }}+\mathbf{r}\right\rangle
$$

for all $\mathbf{v} \in \mathcal{N}$.

Since the incremental problem was assumed to lack solutions, Theorem 2.3 with $\mathbf{D}$ chosen as $\mathbf{M}$ yields the existence of a rigid body motion $\mathbf{w}$ and a contact force $\mathbf{r}$ such that

$$
\left\langle\mathbf{f}^{\text {app }}, \mathbf{v}\right\rangle+\langle\mathbf{r}, \mathbf{v}\rangle=\alpha\langle\mathbf{M w}, \mathbf{v}\rangle .
$$


Let $\mathbf{u}(t)$ be a the time dependent displacement vector. Assuming

$$
\mathbf{u}(t)-\mathbf{u}(0) \in \mathcal{N}
$$

where $\mathcal{N}=\{\mathbf{v}: \mathbf{K v}=\mathbf{0}\}$, let us consider the dynamical problem for the truss exposed to the applied force $\mathbf{f}^{\text {app }}$ and the contact force $\mathbf{r}$. The law of motion (2.14) yields

$$
\left\langle\mathbf{v}, \mathbf{M} \frac{\mathrm{d}^{2} \mathbf{u}(t)}{\mathrm{d} t^{2}}\right\rangle=\left\langle\mathbf{v}, \mathbf{f}^{\text {app }}+\mathbf{r}\right\rangle=\alpha\langle\mathbf{v}, \mathbf{M w}\rangle
$$

for all $\mathbf{v} \in \mathcal{N}$. Since $\frac{\mathrm{d}^{2} \mathbf{u}(t)}{\mathrm{d} t^{2}}, \mathbf{w}$ and $\mathbf{v}$ are vectors in $\mathcal{N}$ and $\mathbf{M}$ is positive definite we conclude from (2.15) that

$$
\frac{\mathrm{d}^{2} \mathbf{u}(t)}{\mathrm{d} t^{2}}=\alpha \mathbf{w} .
$$

Let us solve this equation with the initial conditions

$$
\mathbf{u}(0)=\mathbf{u}_{0}, \quad \frac{\mathrm{d} \mathbf{u}(0)}{\mathrm{d} t}=v_{0} \mathbf{w}
$$

where $\mathbf{u}_{0} \in \mathbb{V}$ and $v_{0}>0$ is a constant. Also, assume $\left(\mathbf{u}_{0}\right)_{i, N}=0$ for all $i \in I_{C}$. The solution of this differential equation is

$$
\mathbf{u}(t)=\mathbf{u}_{0}+\left(v_{0} t+\alpha \frac{t^{2}}{2}\right) \mathbf{w} .
$$

To verify the solution (2.16) we need to prove that this solution is compatible with the contact force field $\mathbf{r}$, and also that the truss never collides with the obstacle so that the solution is smooth and makes sense in equation (2.15). We have

$$
\frac{\mathrm{d} \mathbf{u}(t)_{i, T}}{\mathrm{~d} t}=\left(v_{0}+\alpha t\right) \mathbf{w}_{i, T}=-\lambda_{i}\left(v_{0}+\alpha t\right) \mathbf{r}_{i, T}
$$

that is, $\frac{\mathrm{d} \mathbf{u}(t)}{\mathrm{d} t}$ and $\mathbf{r}$ have opposite direction, as expected. Moreover,

$$
\frac{\mathrm{d} \mathbf{u}(t)_{i, N}}{\mathrm{~d} t}=\left(v_{0}+\alpha t\right) \mathbf{w}_{i, N} \leq 0
$$

so that all nodes are transported away from the obstacles, which implies that no collisions will occur.

Note also that rigid body problems are often statically and dynamically indeterminate systems. But, $\mathbf{u}(t)$ and $\mathbf{r}$ is one solution of this problem.

We have $v_{0} t+\alpha \frac{t^{2}}{2} \geq v_{0} t$, so from (2.16) we find that the a rigid truss may continue to move in the direction of $\mathbf{w}$ if it has a small initial velocity in this direction. We thus find that there is a dynamical solution of the rigid truss problem where the truss is in motion, if the compatibility conditions of Theorem 2.3 are violated. 


\section{Previous results}

By Theorem 2.3 we have the following sufficient condition for a solution to the friction problem (1.5)

$$
\left.\begin{array}{l}
\mathbf{K} \mathbf{w}=\mathbf{0} \\
\alpha\langle\mathbf{D} \mathbf{w}, \mathbf{v}\rangle=\left\langle\mathbf{f}^{\text {app }}, \mathbf{v}\right\rangle+\langle\mathbf{r}, \mathbf{v}\rangle \text { for all } \mathbf{v} \in \mathcal{N} \\
w_{i, N} \leq 0, r_{i, N} \leq 0, w_{i, N} r_{i, N}=0 \\
\left|\mathbf{r}_{i, T}\right| \leq-\mu_{i} r_{i, N} \\
\mathbf{w}_{i, T}=0 \text { if }\left|\mathbf{r}_{i, T}\right|<-\mu_{i} r_{i, N} \\
\mathbf{w}_{i, T}=-\lambda_{i} \mathbf{r}_{i, T} \text { if }\left|\mathbf{r}_{i, T}\right|=-\mu_{i} r_{i, N}>0
\end{array}\right\} \Rightarrow \mathbf{w}=\mathbf{0}
$$

where $\mathcal{N}=\{\mathbf{v}: \mathbf{K v}=\mathbf{0}\}, \mathbf{f}^{\text {app }}$ is the applied force, $\mu_{i}$ is the coefficient of friction, $\alpha \geq 0, \lambda_{i} \geq 0$ and $\mathbf{D}$ is some positive definite matrix.

The inequalities on the left hand side of (2.17) implies

$$
\begin{aligned}
\left\langle\mathbf{f}^{\text {app }}, \mathbf{w}\right\rangle & =\alpha\langle\mathbf{D w}, \mathbf{w}\rangle-\langle\mathbf{r}, \mathbf{w}\rangle \\
& \geq-\langle\mathbf{r}, \mathbf{w}\rangle=-\sum_{i \in I_{C}}\left(r_{i, N} w_{i, N}+\mathbf{r}_{i, T} \cdot \mathbf{w}_{i, T}\right) \\
& =\sum_{i \in I_{C}} \lambda_{i}\left|\mathbf{r}_{i, T}\right|^{2} \geq 0
\end{aligned}
$$

and thus, a weaker condition than (2.17) is

$$
\left.\begin{array}{l}
\mathbf{K} \mathbf{w}=\mathbf{0} \\
w_{i, N} \leq 0 \\
\left\langle\mathbf{f}^{\text {app }}, \mathbf{w}\right\rangle \geq 0
\end{array}\right\} \Rightarrow \mathbf{w}=\mathbf{0}
$$

or equivalently

$$
\left.\begin{array}{l}
\mathbf{K} \mathbf{w}=\mathbf{0} \\
w_{i, N} \leq 0 \\
\mathbf{w} \neq \mathbf{0}
\end{array}\right\} \Rightarrow\left\langle\mathbf{f}^{\text {app }}, \mathbf{w}\right\rangle<0
$$

Hence, if (2.18) or (2.19) are valid, then (2.17) assures the existence of a solution to the friction problem (1.5). The conditions (2.18) and (2.19) are simple, but do not involve the coefficient of friction which makes them restrictive. Note however that we assumed $\mu_{i} \geq \mu_{\text {min }}>0$ for some constant $\mu_{\min }$ when (2.17) was derived.

Sufficient conditions for a solution of problem (1.5) have also been stated by Klarbring et. al. [25]. They study several different friction laws, and state the following condition for three dimensional problems (1.5)

$$
\left.\begin{array}{l}
\mathbf{K} \mathbf{w}=\mathbf{0} \\
w_{i, N} \leq 0
\end{array}\right\} \Rightarrow\left\langle\mathbf{f}^{\text {app }}, \mathbf{w}\right\rangle \leq 0
$$


which does not involve the coefficient of friction, similar to (2.18) and (2.19). For two dimensional friction problems (1.5), Klarbring et. al. [25] use complementarity results to derive the condition (2.17). In fact, they derive a stronger condition which only assumes $\alpha>0$ for the two dimensional case. 


\section{Chapter 3}

\section{Examples of noncoercive friction problems}

This chapter contains examples of noncoercive discrete friction problems that illustrate how Theorem 2.3 can be used to prove sufficient compatibility conditions on the applied force. The general idea is that Theorem 2.3 considers lack of solutions, and thus if we find that the applied force is incompatible with the lack of solutions according to this theorem, then there has to exist a solution to the original friction problem.

\section{Single node}

Let us consider a single node on a flat obstacle to illustrate the use of Theorem 2.3 in a simple example. We will find compatibility conditions on the applied force that are sufficient for the existence of a solution. Figure 3.1 presents the problem and introduces a coordinate frame.

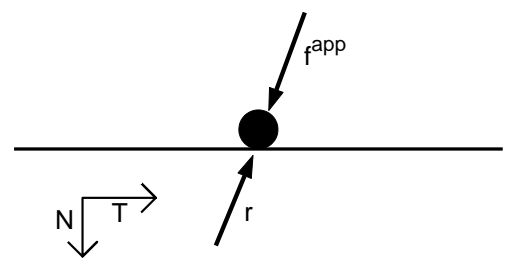

Figure 3.1: A friction problem with a single node. 
3.1. Theorem. There is a solution of the friction problem in Figure 3.1 if

$$
\left\{\begin{array}{c}
f_{N}^{\text {app }}>0 \\
\mu f_{N}^{\text {app }}>\left|\mathbf{f}_{T}^{a p p}\right|
\end{array}\right.
$$

Proof. We prove that (2.10) is prevented if (3.1) is satisfied. Note that $\mathcal{N}=\mathbb{R}^{2}$. We have $\mathbb{V}=\left\{\mathbf{u}: u_{N} \leq 0\right\}$ and choose $\mathbf{D}$ to be the identity matrix. Let us consider the cases $w_{N}=0$ and $w_{N}<0$, where $\mathbf{w}$ is the rigid body motion given by Theorem 2.3 .

I. If $w_{N}<0$ then from (2.11) follows $\mathbf{r}=0$ so (2.10) yields $f_{N}^{\text {app }}=$ $\alpha w_{N} \leq 0$, contradicting (3.1).

II. When $w_{N}=0$, then $(2.10)$ gives $f_{N}^{\text {app }}=-r_{N}$, so that

$$
\begin{aligned}
\left|\mathbf{f}_{T}^{\mathrm{app}}\right| & =\left|\alpha \mathbf{w}_{T}-\mathbf{r}_{T}\right|=\left|-\alpha \lambda \mathbf{r}_{T}-\mathbf{r}_{T}\right| \\
& \geq\left|\mathbf{r}_{T}\right|=-\mu r_{N}=\mu f_{N}^{\text {app }}
\end{aligned}
$$

where we used that $\left|\mathbf{r}_{T}\right|=-\mu r_{N}$ since $\mathbf{w} \neq 0$. Equation (3.2) also contradicts (3.1).

We conclude that (3.1) makes (2.10) impossible, so there exists a solution if the applied force satisfies (3.1) according to the Theorem 2.3. ////

\section{Truss with three nodes}

To give an example on a stiffness matrix and its rigid body displacements, let us consider the truss in Figure 3.2.

Each bar is of length $L$ and has a stiffness $k$. The block vector of displacements is $\mathbf{u}=\left(u_{1 x}, u_{1 y}, u_{2 x}, u_{2 y}, u_{3 x}, u_{3 y}\right)$ and the stiffness matrix is

$$
\mathbf{K}=k\left(\begin{array}{cccccc}
\frac{5}{4} & \frac{\sqrt{3}}{4} & -1 & 0 & -\frac{1}{4} & -\frac{\sqrt{3}}{4} \\
\frac{\sqrt{3}}{4} & \frac{3}{4} & 0 & 0 & -\frac{\sqrt{3}}{4} & -\frac{3}{4} \\
-1 & 0 & \frac{5}{4} & -\frac{\sqrt{3}}{4} & -\frac{1}{4} & \frac{\sqrt{3}}{4} \\
0 & 0 & -\frac{\sqrt{3}}{4} & \frac{3}{4} & \frac{\sqrt{3}}{4} & -\frac{3}{4} \\
-\frac{1}{4} & -\frac{\sqrt{3}}{4} & -\frac{1}{4} & \frac{\sqrt{3}}{4} & \frac{1}{2} & 0 \\
-\frac{\sqrt{3}}{4} & -\frac{3}{4} & \frac{\sqrt{3}}{4} & -\frac{3}{4} & 0 & \frac{3}{2}
\end{array}\right)
$$

A basis for $\mathcal{N}$ is given by

$$
\begin{aligned}
& \mathbf{e}_{x}=\frac{1}{\sqrt{3}}(1,0,1,0,1,0) \\
& \mathbf{e}_{y}=\frac{1}{\sqrt{3}}(0,1,0,1,0,1) \\
& \mathbf{e}_{p}=\frac{1}{\sqrt{3}}\left(\frac{1}{2},-\frac{\sqrt{3}}{2}, \frac{1}{2}, \frac{\sqrt{3}}{2},-1,0\right) .
\end{aligned}
$$


The block vectors $\mathbf{e}_{x}$ and $\mathbf{e}_{y}$ correspond to translations and $\mathbf{e}_{p}$ corresponds to a rotation about the centre $P$. Node 1 and 2 are assumed to be in frictional contact with an obstacle. Sufficient conditions for existence of solutions of the friction problem (1.5) are given in the next section.

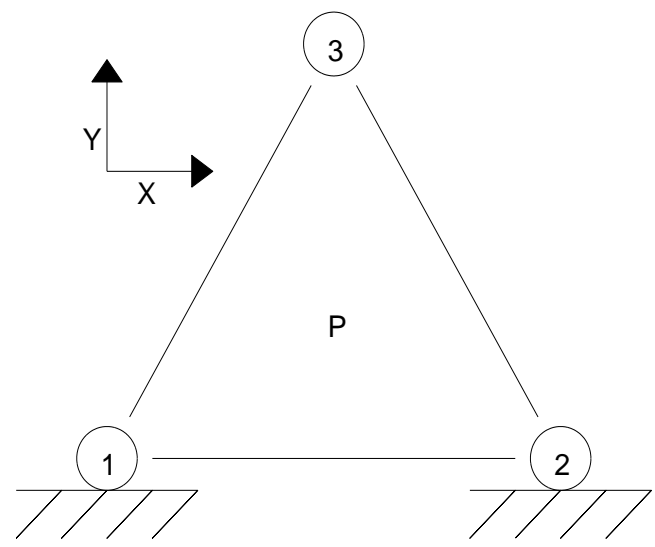

Figure 3.2: A truss consisting of three nodes.

\section{Two dimensional truss on a flat obstacle}

Figure 3.3 illustrates a two dimensional truss of arbitrary shape which is at rest on a flat obstacle with at least two nodes in contact. If only two nodes are in contact, as in Figure 3.3, let us call these nodes node 1 and node 2 . In case more than two nodes are in contact, denote the two nodes in contact which are at the largest distance from each other by node 1 and node 2. The remaining nodes are numbered from 3 to $n$. The point $P$ lies between node 1 and 2 , with equal distance $L$ to both. The friction is assumed to be constant, $\mu_{i}=\mu$ for all $i \in I_{c}$.

We prove the following

3.2. Theorem. A sufficient condition for the existence of a solution to the incremental friction problem presented in Figure 3.3 is

$$
\left\{\begin{array}{c}
F_{y}<0 \\
\left|F_{x}\right|<-\mu F_{y} \\
\left|M_{p}\right|<-L F_{y}
\end{array}\right.
$$

where $F_{x}$ and $F_{y}$ are the resultants of the force $\mathbf{f}^{a p p}$, and $M_{p}$ is the torque caused by $\mathbf{f}^{\text {app }}$ about $P$.

remark: To interpret (3.4) intuitively, the condition $F_{y}<0$ makes the truss remain in contact, the condition $\left|F_{x}\right|<-\mu F_{y}$ is required to prevent 


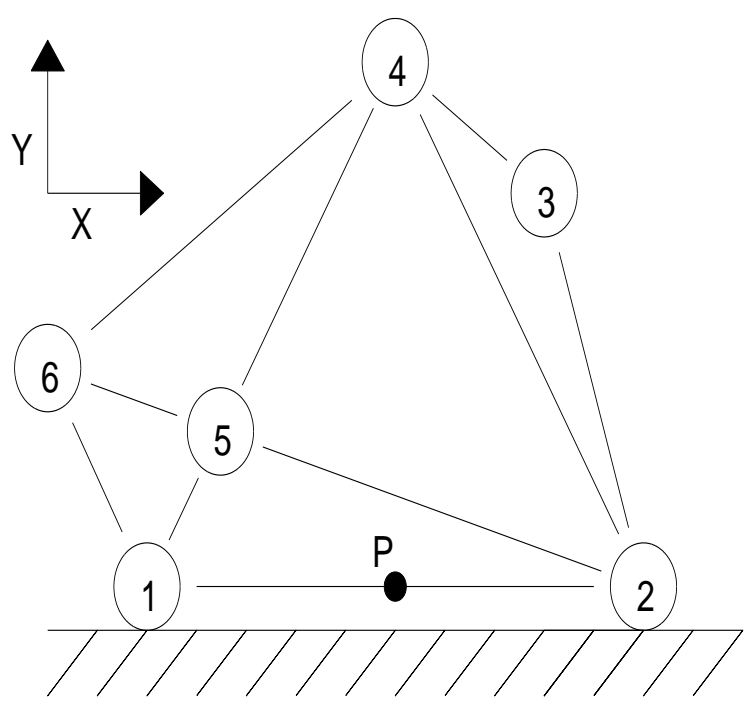

Figure 3.3: Two dimensional truss on a flat obstacle.

the truss from sliding and $\left|M_{p}\right|<-L F_{y}$ prevents the truss from falling over. The last condition can also be stated as a requirement on the line of action of the applied forces, this condition is equivalent to the fact that the line of action have to intersect the contact surface between node 1 and 2 .

Proof. Let us compose a basis of $\mathcal{N}$ by chosing $\mathbf{e}^{x}$ which consists of unit displacements along the $\mathrm{x}$-axis, $\mathbf{e}^{y}$ which consists of unit displacements along the $\mathrm{y}$-axis and finally $\mathbf{e}^{p}$ which is a rotation about $P$, so that $\mathbf{e}_{i}^{p}=$ $\hat{\mathbf{z}} \times \mathbf{q}_{p i}$ where $\hat{\mathbf{z}}$ is a unit vector directed out of Figure 3.3, perpendicular to the $\mathrm{x}$ and y-axes, and $\mathbf{q}_{p i}$ is the position vector of node $i$ relative to $P$. The advantage of this choice of basis is that $F_{x}=\left\langle\mathbf{e}^{x}, \mathbf{f}^{a p p}\right\rangle, F_{y}=\left\langle\mathbf{e}^{y}, \mathbf{f}^{a p p}\right\rangle$ and $M_{p}=\left\langle\mathbf{e}^{p}, \mathbf{f}^{a p p}\right\rangle$, the penalty is that the base is not orthogonal. Decompose $\mathbf{w}$ into components as $\mathbf{w}=w_{x} \mathbf{e}^{x}+w_{y} \mathbf{e}^{y}+w_{p} \mathbf{e}^{p}$.

Since the matrix $\mathbf{D}$ in Theorem 2.3 is at our disposal, let us choose $\mathbf{D}$ as a diagonal matrix so that $\left\langle\mathbf{e}^{y}, \mathbf{D} \mathbf{e}^{p}\right\rangle=0,\left\langle\mathbf{e}^{x}, \mathbf{D} \mathbf{e}^{y}\right\rangle=0$ and $\left\langle\mathbf{e}^{x}, \mathbf{D e}^{p}\right\rangle \leq 0$. That such a matrix can be chosen follows from the fact that $\mathbf{e}_{1}^{y} \cdot \mathbf{e}_{1}^{\bar{p}}=$ $-\frac{L}{2}<0$ and $\mathbf{e}_{2}^{y} \cdot \mathbf{e}_{2}^{p}=\frac{L}{2}>0$, so there are positive numbers $d_{i}$ such that $\left\langle\mathbf{e}^{y}, \mathbf{D} \mathbf{e}^{p}\right\rangle=\sum_{i} d_{i} \mathbf{e}_{i}^{y} \cdot \mathbf{e}_{i}^{p}=0$. Moreover, $\left\langle\mathbf{e}^{x}, \mathbf{D} \mathbf{e}^{y}\right\rangle=0$ and $\left\langle\mathbf{e}^{x}, \mathbf{D e}^{p}\right\rangle \leq 0$ since $\mathbf{e}_{i}^{x} \cdot \mathbf{e}_{i}^{y}=0$ and $\mathbf{e}_{i}^{x} \cdot \mathbf{e}_{i}^{p} \leq 0$ for all nodes $i$.

In the following we consider three possible cases for the action of the displacement $\mathbf{w}$ given by Theorem 2.3 . 
I. First consider the case when both node 1 and 2 leave the contact surface. Then $\mathbf{r}=\mathbf{0}$, so (2.10) with $\mathbf{v}=\mathbf{e}^{y}$ yields

$$
\begin{aligned}
F_{y} & =\left\langle\mathbf{e}^{y}, \mathbf{f}^{a p p}\right\rangle=\alpha\left\langle\mathbf{e}^{y}, \mathbf{w}\right\rangle \\
& =\alpha\left(w_{x}\left\langle\mathbf{e}^{y}, \mathbf{D e}^{x}\right\rangle+w_{y}\left\langle\mathbf{e}^{y}, \mathbf{D e}^{y}\right\rangle+w_{p}\left\langle\mathbf{e}^{y}, \mathbf{D e}^{p}\right\rangle\right) \\
& =\alpha w_{y}\left\langle\mathbf{e}^{y}, \mathbf{D e}^{y}\right\rangle \geq 0
\end{aligned}
$$

which does not agree with (3.4).

II. Next consider the case with only one node in contact, let us assume node 1. Then $0=\mathbf{w}_{1 y}=w_{y} \mathbf{e}_{1 y}^{y}+w_{p} \mathbf{e}_{1 y}^{p}=w_{y}-L w_{p}$. By choosing $\mathbf{v}=\mathbf{e}^{x}, \mathbf{v}=\mathbf{e}^{y}$ and $\mathbf{v}=\mathbf{e}^{p}$ in (2.10) we obtain

$$
\left\{\begin{array}{l}
r_{1 x}+F_{x}=\alpha w_{x}\left\langle\mathbf{e}^{x}, \mathbf{D} \mathbf{e}^{x}\right\rangle+\alpha w_{p}\left\langle\mathbf{e}^{x}, \mathbf{D e}^{p}\right\rangle \\
r_{1 y}+F_{y}=\alpha w_{y}\left\langle\mathbf{e}^{y}, \mathbf{D e}^{y}\right\rangle \\
-L r_{1 y}+M_{p}=\alpha w_{x}\left\langle\mathbf{e}^{p}, \mathbf{D e} \mathbf{e}^{x}\right\rangle+\alpha w_{p}\left\langle\mathbf{e}^{p}, \mathbf{D} \mathbf{e}^{p}\right\rangle
\end{array}\right.
$$

We have $w_{y}>0$ and $w_{p}>0$. First assume also $w_{x} \leq 0$, then from (3.5) follows

$$
\begin{aligned}
L F_{y}+M_{p}= & L \alpha w_{y}\left\langle\mathbf{e}^{y}, \mathbf{D e}^{y}\right\rangle+\alpha w_{x}\left\langle\mathbf{e}^{p}, \mathbf{D e}^{x}\right\rangle \\
& +\alpha w_{p}\left\langle\mathbf{e}^{p}, \mathbf{D e}^{p}\right\rangle \geq 0
\end{aligned}
$$

and thus $M_{p} \geq-L F_{y} \geq 0$, contradicting (3.4). Next assume $w_{x}>0$, then $r_{1 x}=-\mu r_{1 y}$ so that (3.5) give:

$$
\left\{\begin{array}{l}
F_{x}+\mu F_{y}=\alpha w_{x}\left\langle\mathbf{e}^{x}, \mathbf{D e} \mathbf{e}^{x}\right\rangle+\alpha w_{p}\left\langle\mathbf{e}^{x}, \mathbf{D e}^{p}\right\rangle+\mu \alpha w_{y}\left\langle\mathbf{e}^{y}, \mathbf{D} \mathbf{e}^{y}\right\rangle \\
L F_{y}+M_{p}=L \alpha w_{y}\left\langle\mathbf{e}^{y}, \mathbf{D e}^{y}\right\rangle+\alpha w_{x}\left\langle\mathbf{e}^{p}, \mathbf{D e}^{x}\right\rangle+\alpha w_{p}\left\langle\mathbf{e}^{p}, \mathbf{D e}^{p}\right\rangle
\end{array}\right.
$$

and thus

$$
\begin{aligned}
w_{x}\left(F_{x}+\right. & \left.\mu F_{y}\right)+w_{p}\left(L F_{y}+M_{p}\right) \\
= & \alpha \mu w_{x} w_{y}\left\langle\mathbf{e}^{y}, \mathbf{D e}^{y}\right\rangle+\alpha L w_{p} w_{y}\left\langle\mathbf{e}^{y}, \mathbf{D e}^{y}\right\rangle \\
& +\alpha\left\langle w_{p} \mathbf{e}^{p}+w_{x} \mathbf{e}^{x}, \mathbf{D}\left(w_{p} \mathbf{e}^{p}+w_{x} \mathbf{e}^{x}\right)\right\rangle \geq 0
\end{aligned}
$$

so $F_{x}+\mu F_{y} \geq 0$ or $L F_{y}+M_{p} \geq 0$ and hence $F_{x} \geq-\mu F_{y} \geq 0$ or $M_{p} \geq-L F_{y} \geq 0$, contradicting (3.4). The case where node 2 is in contact is analysed similarly.

III. Finally consider the case where both nodes remain in contact. Then $w_{y}=w_{p}=0$ so that $w_{x} \neq 0$ as $\mathbf{w}$ is nonzero, and $\mathbf{v}=\mathbf{e}^{x}$ and $\mathbf{v}=\mathbf{e}^{y}$ in (2.10) we obtain:

$$
\left\{\begin{array}{l}
\sum_{i} r_{i x}+F_{x}=\alpha w_{x}\left\langle\mathbf{e}^{x}, \mathbf{D e}^{x}\right\rangle \\
\sum_{i} r_{i y}+F_{y}=0
\end{array}\right.
$$


Since $w_{x} \neq 0$, we find that $\left|\mu r_{i x}\right|=r_{i y}$ and that $r_{i x}$ and $w_{x}$ have opposite sign. Thus (3.6) yields

$$
\mu\left|F_{x}\right|=\mu\left|\alpha w_{x}\left\langle\mathbf{e}^{x}, \mathbf{D e}^{x}\right\rangle-\sum_{i} r_{i x}\right| \geq\left|\sum_{i} r_{i y}\right|=\left|F_{y}\right|
$$

which again contradicts (3.4).

To sum up, we conclude that (3.4) and (2.10) contradict, and thus Theorem 2.3 establishes that the conditions (3.4) are sufficient to guarantee a solution of the noncoercive friction problem in Figure 3.3.

$/ / /$

\section{Cylinder in a cylindrical groove.}

Let us consider a cylindrical truss in a cylindrical groove, which is illustrated in Figure 3.4. The cylinder has radius $R$ and length $2 L$, and the friction is assumed to be constant in each contact node, $\mu_{i}=\mu$ for all $i \in I_{c}$. Introduce a coordinate frame and node 1 to 6 as displayed in Figure 3.5. The origin of the coordinate frame is at the geometrical symmetry axis of the cylinder. Additional nodes are numbered from 7 to $n$. Use the block vectors $\mathbf{e}^{x}, \mathbf{e}^{y}$ and $\mathbf{e}^{z}$ to denote unit displacements for each node in the $x, y$ and $z$-direction, and $\mathbf{e}^{x x}, \mathbf{e}^{y y}$ and $\mathbf{e}^{z z}$ to denote a rotation about respective axis, so that $\mathbf{e}_{i}^{x}=\hat{\mathbf{x}}$ and $\mathbf{e}_{i}^{x x}=\hat{\mathbf{x}} \times \mathbf{q}_{i}$ where $\hat{\mathbf{x}}$ is a unit vector directed along the $\mathrm{x}$-axis and $\mathbf{q}_{i}$ is the position vector of node $i$ relative to the origin of the coordinate frame. The resultants of the applied force $\mathbf{f}^{a p p}$ are denoted $F_{x}, F_{y}$ and $F_{z}$ and the resulting torques are $M_{x}, M_{y}$ and $M_{z}$. Thus $F_{x}=\left\langle\mathbf{e}^{x}, \mathbf{f}^{a p p}\right\rangle, F_{y}=\left\langle\mathbf{e}^{y}, \mathbf{f}^{a p p}\right\rangle$ and $F_{z}=\left\langle\mathbf{e}^{z}, \mathbf{f}^{a p p}\right\rangle$, and also $M_{x}=\left\langle\mathbf{e}^{x x}, \mathbf{f}^{a p p}\right\rangle, M_{y}=\left\langle\mathbf{e}^{y y}, \mathbf{f}^{a p p}\right\rangle$ and $M_{z}=\left\langle\mathbf{e}^{z z}, \mathbf{f}^{a p p}\right\rangle$.

The rigid body motion $\mathbf{w}$ can be uniquely decomposed in components, $\mathbf{w}=w_{x} \mathbf{e}^{x}+w_{y} \mathbf{e}^{y}+w_{z} \mathbf{e}^{z}+w_{x x} \mathbf{e}^{x x}+w_{y y} \mathbf{e}^{y y}+w_{z z} \mathbf{e}^{z z}$. Due to the existence of nodes 1 to 6 , we have $w_{y}=0$ and $w_{z z}=0$, and also $\left|w_{y y}\right| \leq L w_{z}$. Since

$$
\begin{array}{ll}
\mathbf{e}_{1}^{z} \cdot \mathbf{e}_{1}^{x x}>0, & \mathbf{e}_{1}^{y y} \cdot \mathbf{e}_{1}^{x x}<0 \\
\mathbf{e}_{3}^{z} \cdot \mathbf{e}_{3}^{x x}<0, & \mathbf{e}_{3}^{y y} \cdot \mathbf{e}_{3}^{x x}>0 \\
\mathbf{e}_{4}^{z} \cdot \mathbf{e}_{4}^{x x}>0, & \mathbf{e}_{4}^{y y} \cdot \mathbf{e}_{4}^{x x}>0 \\
\mathbf{e}_{6}^{z} \cdot \mathbf{e}_{6}^{x x}<0, & \mathbf{e}_{6}^{y y} \cdot \mathbf{e}_{6}^{x x}<0
\end{array}
$$

it is possible to choose the diagonal elements $d_{1}, d_{3}, d_{4}$ and $d_{6}$ of a positive diagonal matrix $\mathbf{D}$ so that $\left\langle\mathbf{e}^{z}, \mathbf{D} \mathbf{e}^{x x}\right\rangle=0$ and $\left\langle\mathbf{e}^{y y}, \mathbf{D} \mathbf{e}^{x x}\right\rangle=0$.

Let us first prove the following:

3.3. Lemma. Assume $w_{z}>0$ and define

$$
a=\left\{\begin{array}{l}
w_{x} \text { if } w_{x}=0 \text { or }\left|w_{x}\right| \leq R\left|w_{y y}\right| \\
\frac{R\left|w_{y y}\right| w_{x}}{\left|w_{x}\right|} \text { otherwise }
\end{array}\right.
$$




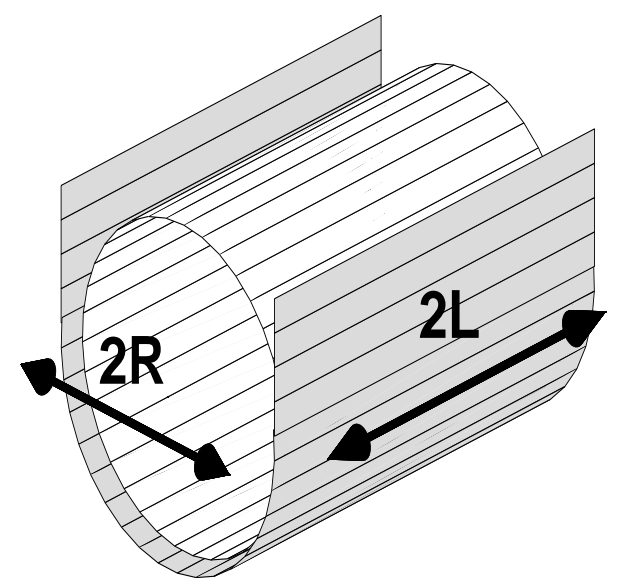

Figure 3.4: A cylinder in a cylindrical groove.

and

$$
b=\left\{\begin{array}{l}
w_{x x} \text { if } w_{x x}=0 \text { or }\left|w_{x x}\right| \leq \frac{2}{R}\left|w_{z}\right| \\
\frac{2\left|w_{z}\right| w_{x x}}{R\left|w_{x x}\right|} \text { otherwise }
\end{array}\right.
$$

and let

$$
\hat{\mathbf{w}}=a \mathbf{e}^{x}+w_{z} \mathbf{e}^{z}+b \mathbf{e}^{x x}+w_{y y} \mathbf{e}^{y y}
$$

Then

I. $\hat{\mathbf{w}}_{i} \cdot \mathbf{w}_{i} \geq 0$ for any node $i$ such that $\left|\mathbf{e}_{i}^{x x}\right|=R$.

II. the following inequality

$$
\begin{aligned}
\hat{\mathbf{w}}_{i} \cdot \mathbf{w}_{i} \geq & w_{z}\left(b+w_{x x}\right)\left(\mathbf{e}_{i}^{z} \cdot \mathbf{e}_{i}^{x x}\right) \\
& +w_{y y}\left(b+w_{x x}\right)\left(\mathbf{e}_{i}^{y y} \cdot \mathbf{e}_{i}^{x x}\right)
\end{aligned}
$$

is valid for every node $i$ of the truss.

III. $\langle\hat{\mathbf{w}}, \mathbf{D w}\rangle \geq 0$.

$I V .\langle\hat{\mathbf{w}}, \mathbf{r}\rangle \leq 0$, where $\mathbf{r}$ is the contact force that correspond to $\mathbf{w}$ according to Theorem 2.3.

Proof. $\quad$ I. Decompose $\mathbf{e}_{i}^{y y}$ in $x$ and $z$-component, $\mathbf{e}_{i}^{y y}=\mathbf{e}_{i, x}^{y y}+\mathbf{e}_{i, z}^{y y}$. We 


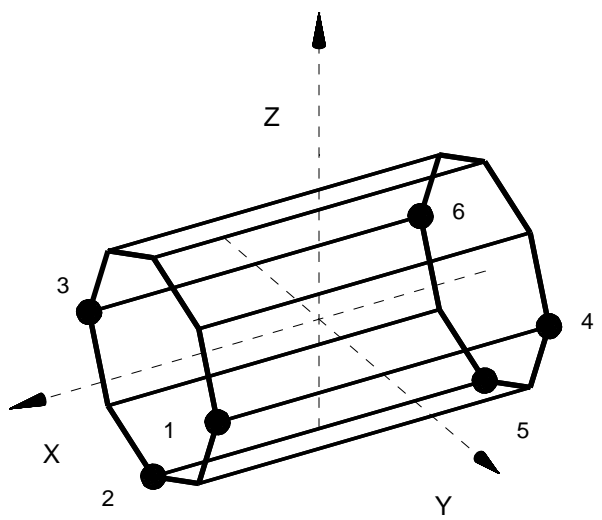

Figure 3.5: Coordinate frame and node numbering of the cylinder.

obtain

$$
\begin{aligned}
\hat{\mathbf{w}}_{i} \cdot \mathbf{w}_{i}= & \left(a \mathbf{e}_{i}^{x}+w_{z} \mathbf{e}_{i}^{z}+b \mathbf{e}_{i}^{x x}+w_{y y} \mathbf{e}_{i}^{y y}\right) \\
& \cdot\left(w_{x} \mathbf{e}_{i}^{x}+w_{z} \mathbf{e}_{i}^{z}+w_{x x} \mathbf{e}_{i}^{x x}+w_{y y} \mathbf{e}_{i}^{y y}\right) \\
= & {\left[a w_{x}+w_{y y}^{2}\left|\mathbf{e}_{i, x}^{y y}\right|^{2}+\left(w_{x} w_{y y}+a w_{y y}\right)\left(\mathbf{e}_{i, x}^{y y} \cdot \mathbf{e}_{i}^{x}\right)\right] } \\
& +\left[w_{z}^{2}+b w_{x x}\left|\mathbf{e}_{i}^{x x}\right|^{2}+w_{y y}^{2}\left|\mathbf{e}_{i, z}^{y y}\right|^{2}\right. \\
& +\left(w_{z} b+w_{z} w_{y y}\right)\left(\mathbf{e}_{i}^{z} \cdot \mathbf{e}_{i}^{x x}\right) \\
& \left.+2 w_{z} w_{y y}\left(\mathbf{e}_{i}^{z} \cdot \mathbf{e}_{i}^{y y}\right)+\left(w_{x x} w_{y y}+b w_{y y}\right)\left(\mathbf{e}_{i}^{x x} \cdot \mathbf{e}_{i}^{y y}\right)\right]
\end{aligned}
$$

We prove that both expressions within square brackets are positive. Let us first consider the first square bracket. If $a=w_{x}$, then $\left|w_{x}\right| \leq$ $R\left|w_{y y}\right|$ and we obtain

$$
\begin{aligned}
{\left[a w_{x}\right.} & \left.+w_{y y}^{2}\left|\mathbf{e}_{i, x}^{y y}\right|^{2}+\left(w_{x} w_{y y}+a w_{y y}\right)\left(\mathbf{e}_{i, x}^{y y} \cdot \mathbf{e}_{i}^{x}\right)\right] \\
& =\left(w_{x} \mathbf{e}_{i}^{x}+w_{y y} \mathbf{e}_{i, x}^{y y}\right) \cdot\left(w_{x} \mathbf{e}_{i}^{x}+w_{y y} \mathbf{e}_{i, x}^{y y}\right) \geq 0
\end{aligned}
$$

and if $a=\frac{R\left|w_{y y}\right| w_{x}}{\left|w_{x}\right|}$, then $\left|w_{x}\right|>R\left|w_{y y}\right|$ so that

$$
\begin{aligned}
{\left[a w_{x}\right.} & \left.+w_{y y}^{2}\left|\mathbf{e}_{i, x}^{y y}\right|^{2}+\left(w_{x} w_{y y}+a w_{y y}\right)\left(\mathbf{e}_{i, x}^{y y} \cdot \mathbf{e}_{i}^{x}\right)\right] \\
& \geq R\left|w_{x}\right|\left|w_{y y}\right|+\left|w_{y y}\right|^{2}\left|\mathbf{e}_{i, x}^{y y}\right|^{2}-\left|w_{x}\right|\left|w_{y y}\right|\left|\mathbf{e}_{i, x}^{y y}\right|-R\left|w_{y y}\right|^{2}\left|\mathbf{e}_{i, x}^{y y}\right| \\
& =\left|w_{y y}\right|\left(\left|w_{x}\right|-\left|w_{y y}\right|\left|\mathbf{e}_{i, x}^{y y}\right|\right)\left(R-\left|\mathbf{e}_{i, x}^{y y}\right|\right) \geq 0
\end{aligned}
$$


where $\left|\mathbf{e}_{i, x}^{y y}\right| \leq R$ was used to conclude the last inequality. This proves that the first square bracket in (3.9) is positive.

Now let us prove the second square bracket in (3.9) is positive. Define $\eta_{i}=w_{z} \mathbf{e}_{i}^{z}+w_{y y} \mathbf{e}_{i, z}^{y y}$, then we obtain

$$
\begin{aligned}
{\left[w_{z}^{2}\right.} & +b w_{x x}\left|\mathbf{e}_{i}^{x x}\right|^{2}+w_{y y}^{2}\left|\mathbf{e}_{i, z}^{y y}\right|^{2}+\left(w_{z} b+w_{z} w_{y y}\right)\left(\mathbf{e}_{i}^{z} \cdot \mathbf{e}_{i}^{x x}\right) \\
& \left.+2 w_{z} w_{y y}\left(\mathbf{e}_{i}^{z} \cdot \mathbf{e}_{i}^{y y}\right)+\left(w_{x x} w_{y y}+b w_{y y}\right)\left(\mathbf{e}_{i}^{x x} \cdot \mathbf{e}_{i}^{y y}\right)\right] \\
& =\left(b \mathbf{e}_{i}^{x x}+w_{z} \mathbf{e}_{i}^{z}+w_{y y} \mathbf{e}_{i, z}^{y y}\right) \cdot\left(w_{x x} \mathbf{e}_{i}^{x x}+w_{z} \mathbf{e}_{i}^{z}+w_{y y} \mathbf{e}_{i, z}^{y y}\right) \\
& =\left(b \mathbf{e}_{i}^{x x}+\eta_{i}\right) \cdot\left(w_{x x} \mathbf{e}_{i}^{x x}+\eta_{i}\right)
\end{aligned}
$$

where we used $\mathbf{e}_{i}^{z} \cdot \mathbf{e}_{i}^{y y}=\mathbf{e}_{i}^{z} \cdot \mathbf{e}_{i, z}^{y y}$ and $\mathbf{e}_{i}^{x x} \cdot \mathbf{e}_{i}^{y y}=\mathbf{e}_{i}^{x x} \cdot \mathbf{e}_{i, z}^{y y}$. It follows that (3.10) is positive if $b=w_{x x}$, it remains to consider the case $b=\frac{2\left|w_{z}\right| w_{x x}}{R\left|w_{x x}\right|}$, which also means $\left|w_{x x}\right|>\frac{2}{R}\left|w_{z}\right|$. We obtain

$$
\left|\eta_{i}\right| \leq\left|w_{z}\right|+L\left|w_{y y}\right| \leq 2\left|w_{z}\right|=2\left|w_{z}\right| \frac{\left|\mathbf{e}_{i}^{x x}\right|}{R}=\left|b \mathbf{e}_{i}^{x x}\right|
$$

and

$$
\left|\eta_{i}\right| \leq\left|w_{z}\right|+L\left|w_{y y}\right| \leq 2\left|w_{z}\right|=2\left|w_{z}\right| \frac{\left|\mathbf{e}_{i}^{x x}\right|}{R} \leq\left|w_{x x} \mathbf{e}_{i}^{x x}\right|
$$

so that

$$
\begin{aligned}
\left(b \mathbf{e}_{i}^{x x}\right. & \left.+\eta_{i}\right) \cdot\left(w_{x x} \mathbf{e}_{i}^{x x}+\eta_{i}\right) \\
& \geq b w_{x x}\left|\mathbf{e}_{i}^{x x}\right|^{2}+\left|\eta_{i}\right|^{2}-\left|\eta_{i}\right|\left(\left|w_{x x} \mathbf{e}_{i}^{x x}\right|+\left|b \mathbf{e}_{i}^{x x}\right|\right) \\
& =\left(\left|w_{x x} \mathbf{e}_{i}^{x x}\right|-\left|\eta_{i}\right|\right)\left(\left|b \mathbf{e}_{i}^{x x}\right|-\left|\eta_{i}\right|\right) \geq 0
\end{aligned}
$$

From (3.10) and (3.11) it follows that the second bracket is positive. Thus we can conclude $\hat{\mathbf{w}}_{i} \cdot \mathbf{w}_{i} \geq 0$ from (3.9).

II. We use (3.9), and recall from the proof of (I) that the first square bracket is positive. Note also $b w_{x x} \geq 0$. We obtain

$$
\begin{aligned}
\hat{\mathbf{w}}_{i} \cdot \mathbf{w}_{i}= & \left(a \mathbf{e}_{i}^{x}+w_{z} \mathbf{e}_{i}^{z}+b \mathbf{e}_{i}^{x x}+w_{y y} \mathbf{e}_{i}^{y y}\right) \\
& \cdot\left(w_{x} \mathbf{e}_{i}^{x}+w_{z} \mathbf{e}_{i}^{z}+w_{x x} \mathbf{e}_{i}^{x x}+w_{y y} \mathbf{e}_{i}^{y y}\right) \\
\geq & {\left[w_{z}^{2}+b w_{x x}\left|\mathbf{e}_{i}^{x x}\right|^{2}+w_{y y}^{2}\left|\mathbf{e}_{i, z}^{y y}\right|^{2}\right.} \\
& +\left(w_{z} b+w_{z} w_{y y}\right)\left(\mathbf{e}_{i}^{z} \cdot \mathbf{e}_{i}^{x x}\right)+2 w_{z} w_{y y}\left(\mathbf{e}_{i}^{z} \cdot \mathbf{e}_{i}^{y y}\right) \\
& \left.+\left(w_{x x} w_{y y}+b w_{y y}\right)\left(\mathbf{e}_{i}^{x x} \cdot \mathbf{e}_{i}^{y y}\right)\right] \\
\geq & \left|w_{z}\right|^{2}+w_{y y}^{2}\left|\mathbf{e}_{i, z}^{y y}\right|^{2}-2\left|w_{z}\right|\left|w_{y y}\right|\left|\mathbf{e}_{i, z}^{y y}\right| \\
& +\left(w_{z} b+w_{z} w_{y y}\right)\left(\mathbf{e}_{i}^{z} \cdot \mathbf{e}_{i}^{x x}\right) \\
& +\left(w_{x x} w_{y y}+b w_{y y}\right)\left(\mathbf{e}_{i}^{x x} \cdot \mathbf{e}_{i}^{y y}\right) \\
= & \left(\left|w_{z}\right|-\left|w_{y y}\right|\left|\mathbf{e}_{i, z}^{y y}\right|\right)^{2}+\left(w_{z} b+w_{z} w_{y y}\right)\left(\mathbf{e}_{i}^{z} \cdot \mathbf{e}_{i}^{x x}\right) \\
& +\left(w_{x x} w_{y y}+b w_{y y}\right)\left(\mathbf{e}_{i}^{x x} \cdot \mathbf{e}_{i}^{y y}\right)
\end{aligned}
$$


and this proves (II).

III. The choice of $\mathbf{D}$ implies

$$
0=\left\langle\mathbf{e}^{z}, \mathbf{D} \mathbf{e}^{x x}\right\rangle=\sum_{i} d_{i}\left(\mathbf{e}_{i}^{z} \cdot \mathbf{e}_{i}^{x x}\right)
$$

and

$$
0=\left\langle\mathbf{e}^{y y}, \mathbf{D} \mathbf{e}^{x x}\right\rangle=\sum_{i} d_{i}\left(\mathbf{e}_{i}^{y y} \cdot \mathbf{e}_{i}^{x x}\right)
$$

where $d_{i}$ are diagonal elements of D. From (II) we obtain

$$
\begin{aligned}
\langle\hat{\mathbf{w}}, \mathbf{D w}\rangle= & \sum_{i} d_{i}\left(\hat{\mathbf{w}}_{i} \cdot \mathbf{w}_{i}\right) \\
\geq & w_{z}\left(b+w_{x x}\right) \sum_{i} d_{i}\left(\mathbf{e}_{i}^{z} \cdot \mathbf{e}_{i}^{x x}\right) \\
& +w_{y y}\left(b+w_{x x}\right) \sum_{i} d_{i}\left(\mathbf{e}_{i}^{y y} \cdot \mathbf{e}_{i}^{x x}\right)=0
\end{aligned}
$$

IV. Consider any contact node $i$ in contact. Since $\mathbf{e}_{i}^{x}$ and $\mathbf{e}_{i}^{x x}$ lack normal components, we obtain $\hat{w}_{i, N}=w_{i, N}$. Thus $\hat{w}_{i, N} r_{i, N}=0$ by Theorem 2.3. We also have $\mathbf{w}_{i, T}=-\lambda_{i} \mathbf{r}_{i, T}$ from 2.11, and $\left|\mathbf{e}_{i}^{x x}\right|=R$ for contact nodes so (I) yields

$$
\langle\hat{\mathbf{w}}, \mathbf{r}\rangle=\sum_{i} \hat{\mathbf{w}}_{i, T} \cdot \mathbf{r}_{i, T}=\sum_{i}-\frac{\hat{\mathbf{w}}_{i, T} \cdot \mathbf{w}_{i, T}}{\lambda_{i}} \leq 0 .
$$

where the last summation is performed over all $i$ such that $\left|\mathbf{r}_{i, T}\right|>0$ so that $\lambda_{i}>0$.

Now, let us prove an existence result for the cylinder:

3.4. Theorem. The following condition is sufficient for the existence of a solution to the incremental friction problem of the cylinder:

$$
\left\{\begin{array}{c}
F_{z}<0 \\
\frac{1}{L}\left|M_{y}\right|+\frac{2}{R}\left|M_{x}\right|+\frac{R}{L}\left|F_{x}\right|<-F_{z} \\
\frac{1}{\mu}\left|F_{x}\right|+\left(1+\frac{1}{\mu}\right) \frac{\left|M_{x}\right|}{R}<-F_{z}
\end{array}\right.
$$

Proof. Let us apply Theorem 2.3. We consider the cases $w_{z}=0$ and $w_{z}>0$ separately.

If $w_{z}>0$, let us use $\hat{\mathbf{w}}$ from Lemma 3.3 in (2.10) to obtain

$$
a F_{x}+w_{z} F_{z}+b M_{x}+w_{y y} M_{y}=\left\langle\hat{\mathbf{w}}, \mathbf{f}^{\text {app }}\right\rangle=\alpha\langle\hat{\mathbf{w}}, \mathbf{D} \mathbf{w}\rangle-\langle\hat{\mathbf{w}}, \mathbf{r}\rangle \geq 0
$$


so that

$$
\begin{aligned}
0 & \leq-F_{z} \leq \frac{|a|}{w_{z}}\left|F_{x}\right|+\frac{\left|w_{y y}\right|}{w_{z}}\left|M_{y}\right|+\frac{|b|}{w_{z}}\left|M_{x}\right| \\
& \leq \frac{R}{L}\left|F_{x}\right|+\frac{1}{L}\left|M_{y}\right|+\frac{2}{R}\left|M_{x}\right|
\end{aligned}
$$

since $\frac{|a|}{w_{z}}=\frac{|a|}{w_{y y}} \frac{w_{y y}}{w_{z}} \leq R \frac{1}{L}$ if $w_{y y} \neq 0$, and this contradicts (3.13).

Next, consider the case when $w_{z}=0$, then also $w_{y y}=0$. Use $\mathbf{e}^{z}$ in (2.10), then we obtain

$$
F_{z}+\sum_{i} \mathbf{r}_{i, z}=\alpha\left(w_{x}\left\langle\mathbf{e}^{z}, \mathbf{D} \mathbf{e}^{x}\right\rangle+w_{x x}\left\langle\mathbf{e}^{z}, \mathbf{D} \mathbf{e}^{x x}\right\rangle\right)=0
$$

Use $\mathbf{e}^{x x}$ in (2.10), and note that $w_{x x}$ and $\left\langle\mathbf{e}^{x x}, \mathbf{r}\right\rangle$ have opposite sign, to obtain

$$
\begin{aligned}
\left|M_{x}\right| & =\left|\left\langle\mathbf{e}^{x x}, \mathbf{f}^{\text {app }}\right\rangle\right|=\left|-\left\langle\mathbf{e}^{x x}, \mathbf{r}\right\rangle+\alpha w_{x x}\left\langle\mathbf{e}^{x x}, \mathbf{D} \mathbf{e}^{x x}\right\rangle\right| \\
& \geq\left|\left\langle\mathbf{e}^{x x}, \mathbf{r}\right\rangle\right|=R \sum_{i}\left|\mathbf{r}_{i, T y z}\right|
\end{aligned}
$$

where $\mathbf{r}_{i, T y z}$ is the component in the $y z$-plane of the tangential contact force. The last equality in (3.14) assumed $w_{x x} \neq 0$, and the inequality used that $w_{x x}$ and $\left\langle\mathbf{e}^{x x}, \mathbf{r}\right\rangle$ have opposite sign. Finally, use $\mathbf{e}^{x}$ in (2.10) to obtain

$$
\begin{aligned}
\left|F_{x}\right| & \geq\left|\left\langle\mathbf{e}^{x}, \mathbf{f}^{\text {app }}\right\rangle\right|=\left|-\left\langle\mathbf{e}^{x}, \mathbf{r}\right\rangle+\alpha w_{x}\left\langle\mathbf{e}^{x}, \mathbf{D e}^{x}\right\rangle\right| \\
\geq\left|-\left\langle\mathbf{e}^{x}, \mathbf{r}\right\rangle\right| & =\sum_{i}\left|\mathbf{r}_{i, x}\right| .
\end{aligned}
$$

The last equality of (3.15) assumed $w_{x} \neq 0$.

If $w_{x} \neq 0$ and $w_{x x} \neq 0$ then we obtain

$$
\begin{aligned}
\left|F_{z}\right| & \leq \sum_{i}\left|\mathbf{r}_{i, z}\right| \leq \sum_{i}\left(\left|r_{i, N}\right|+\left|r_{i, T y z}\right|\right)=\sum_{i}\left(\frac{1}{\mu}\left|r_{i, T}\right|+\left|r_{i, T y z}\right|\right) \\
& \leq \sum_{i}\left(\frac{1}{\mu}\left|r_{i, x}\right|+\left(1+\frac{1}{\mu}\right)\left|r_{i, T y z}\right|\right) \leq \frac{1}{\mu}\left|F_{x}\right|+\left(1+\frac{1}{\mu}\right) \frac{\left|M_{x}\right|}{R}
\end{aligned}
$$

which contradicts (3.13). If $w_{x}=0$ and $w_{x x} \neq 0$ then

$$
\begin{aligned}
\left|F_{z}\right| & \leq \sum_{i}\left|\mathbf{r}_{i, z}\right| \leq \sum_{i}\left(\left|r_{i, N}\right|+\left|r_{i, T y z}\right|\right) \\
& =\sum_{i}\left(\frac{1}{\mu}\left|r_{i, T y z}\right|+\left|r_{i, T y z}\right|\right) \leq\left(1+\frac{1}{\mu}\right) \frac{\left|M_{x}\right|}{R}
\end{aligned}
$$

and if $w_{x} \neq 0$ and $w_{x x}=0$ then

$$
\begin{aligned}
\left|F_{z}\right| & \leq \sum_{i}\left|\mathbf{r}_{i, z}\right| \leq \sum_{i}\left(\left|r_{i, N}\right|+\left|r_{i, T y z}\right|\right) \\
& =\sum_{i}\left(\frac{1}{\mu}\left|r_{i, x}\right|+\left|r_{i, T y z}\right|\right) \leq \frac{1}{\mu}\left|F_{x}\right|+\frac{\left|M_{x}\right|}{R}
\end{aligned}
$$


which also contradicts (3.13).

This proves that (3.13) provides sufficient conditions for the existence of a solution of the cylindrical incremental friction problem.

remark: This result is possible to improve. Consider for example the case $w_{x} \neq 0$ and $w_{x x} \neq 0$. Above we have used the triangle inequality: $\left|\mathbf{r}_{i, T}\right| \leq\left|\mathbf{r}_{i, x}\right|+\left|\mathbf{r}_{i, T y z}\right|$. Instead, using pytaghora's theorem one obtains

$$
\left|\mathbf{r}_{i, T}\right|=\sqrt{\left|\mathbf{r}_{i, x}\right|^{2}+\left|\mathbf{r}_{i, T y z}\right|^{2}} .
$$

Since the ratio $\frac{\left|\mathbf{r}_{i, x}\right|}{\left|\mathbf{r}_{i, \text { Tyz }}\right|}=\frac{\left|\mathbf{w}_{i, x}\right|}{\left|\mathbf{w}_{i, \text { Tyz }}\right|}$ is constant, one finds

$$
\sum_{i} \sqrt{\left|\mathbf{r}_{i, x}\right|^{2}+\left|\mathbf{r}_{i, T y z}\right|^{2}}=\sqrt{\left(\sum_{i}\left|\mathbf{r}_{i, x}\right|\right)^{2}+\left(\sum_{i}\left|\mathbf{r}_{i, T y z}\right|\right)^{2}} .
$$

Proceeding as above, we find that the condition $\frac{1}{\mu}\left|F_{x}\right|+\left(1+\frac{1}{\mu}\right) \frac{\left|M_{x}\right|}{R}<-F_{z}$ can be replaced by $\frac{1}{\mu} \sqrt{\left|F_{x}\right|^{2}+\left(\frac{\left|M_{x}\right|}{R}\right)^{2}}+\frac{\left|M_{x}\right|}{R}<-F_{z}$. 


\section{Chapter 4}

\section{Corollaries from the existence theorem}

In this section we derive some properties of the solutions to the incremental friction problem where the applied force fulfills the compatibility condition in Theorem 2.3. The ideas of proof are similar as in the proof of Theorem 2.3. Let us first derive the following generalization of Lemma 2.2 (III):

4.1. Lemma. Assume $\mathbf{f}^{n}$ is a bounded sequence of forces, that is $\left|\mathbf{f}^{n}\right| \leq C_{4}$ for some constant $C_{4}$, and assume also that there exist solutions to the friction problem (1.5) or (1.7) for all $\mathbf{f}^{n}$ and for a fixed $\mathbf{u}^{\mathrm{ref}}$. Then the sequence $\left|\mathbf{r}^{n}\right|$ is also bounded.

Proof. This proof is similar to the proof of Lemma 2.2 (III). Let us assume $\left|\mathbf{r}^{n}\right| \rightarrow \infty$ to obtain a contradiction. Then $\left|\mathbf{u}^{n}\right| \rightarrow \infty$, since $\mathbf{r}^{n}=\mathbf{K} \mathbf{u}^{n}-\mathbf{f}^{n}$ and $\mathbf{f}^{n}$ is bounded .

Let $\mathbf{R}^{n}$ denote $\mathbf{R}^{n}=\mathbf{K} \mathbf{u}^{n}=\mathbf{r}^{n}+\mathbf{f}^{n}$, then $\left|\mathbf{R}^{n}\right| \geq\left|\mathbf{r}^{n}\right|-\left|\mathbf{f}^{n}\right| \rightarrow \infty$ so we can assume $\left|\mathbf{R}^{n}\right|>0$ for all $n$. Let $\mathbf{x}^{n}=\mathbf{R}^{n} /\left|\mathbf{R}^{n}\right|$, then $\mathbf{r}^{n}=\left|\mathbf{R}^{n}\right| \mathbf{x}^{n}-\mathbf{f}^{n}$.

We have $\left|\mathbf{x}^{n}\right|=1$ and $\mathbf{x}^{n} \in \mathcal{R}$, so that we can assume convergence, $\mathbf{x}^{n} \rightarrow \mathbf{x} \in \mathcal{R}$, where $\mathcal{R}$ is the range of $\mathbf{K}$. If $\left|\mathbf{x}_{i}\right| \neq 0$, then

$$
\lim _{n \rightarrow \infty} \frac{\mathbf{r}_{i}^{n}}{\left|\mathbf{r}_{i}^{n}\right|}=\sigma_{i} \mathbf{x}_{i}
$$

where $\sigma_{i} \geq 1$.

To complete this proof, we proceed as in the proof of Lemma 2.2 (III).

\section{Boundedness of the solution}

The compatibility conditions of Theorem 2.3 do not only imply the existence of a solution, but we can also prove that in case there are several 
solutions, then we have an upper bound on their norm. This is the meaning of the next theorem.

4.2. Theorem. The compatibility condition on the applied force in Theorem 2.3 implies that the set of solutions is bounded, i.e. there is a constant $C_{5}$ such that $|\mathbf{u}| \leq C_{5}$ for all solutions $\mathbf{u}$.

Proof. Assume on the contrary that no such constant $C_{5}$ exists, Then there exists a sequence of solutions $\left\{\mathbf{u}^{n}\right\}$ such that $\left|\mathbf{u}^{n}\right| \rightarrow \infty$. Lemma 4.1 implies that $\mathbf{r}^{n}$ is bounded, so we can assume convergence $\mathbf{r}^{n} \rightarrow \mathbf{r}$. We obtain

$$
0=\left\langle\mathbf{K u}^{n}, \mathbf{v}\right\rangle=\langle\mathbf{f}, \mathbf{v}\rangle+\left\langle\mathbf{r}^{n}, \mathbf{v}\right\rangle \rightarrow\langle\mathbf{f}, \mathbf{v}\rangle+\langle\mathbf{r}, \mathbf{v}\rangle
$$

where $\mathbf{v} \in \mathcal{N}$, and therefore

$$
\langle\mathbf{f}, \mathbf{v}\rangle+\langle\mathbf{r}, \mathbf{v}\rangle=0
$$

for all $\mathbf{v} \in \mathcal{N}$.

Moreover, let us define $\mathbf{w}^{n}$ as in Lemma 2.2, and also assume convergence $\mathbf{w}^{n} \rightarrow \mathbf{w}$ as $\mathbf{w}^{n}$ are bounded, then

$$
\left|\mathbf{u}^{n}-\mathbf{u}_{T}^{\mathrm{ref}}\right| \mathbf{K} \mathbf{w}^{n}=\mathbf{f}+\mathbf{r}^{n}-\mathbf{K} \mathbf{u}_{T}^{\mathrm{ref}}
$$

and since the right hand side is bounded and $\left|\mathbf{u}^{n}-\mathbf{u}_{T}^{\text {ref }}\right| \rightarrow \infty$ we see that $\mathbf{K w}=0$, so that $\mathbf{w} \in \mathcal{N}$.

To prove the correspondence between $\mathbf{w}$ and $\mathbf{r}$ stated in the conditions of Theorem 2.3 one only proceeds as in the proof of the Theorem 2.3. So we conclude that if there is no bound on the set of solutions, then there exist $\mathbf{w}$ and $\mathbf{r}$ as in the case when the friction problem lacks solutions according to Theorem 2.3.

remark: This theorem states that the Theorem 2.3 is not able to determine the existence of solutions when these solutions are not bounded. For instance, consider the single node in example 1 chapter 3 . There are also solutions when

$$
\left\{\begin{array}{c}
f_{N}>0 \\
\mu f_{N}=\left|\mathbf{f}_{T}\right|
\end{array}\right.
$$

for this single node example. One set of solutions are $u_{N}=0, u_{T}=\frac{\tau \mathbf{f}_{T}}{\left|\mathbf{f}_{T}\right|}$ and $\mathbf{r}=-\mathbf{f}$ where $\tau \geq 0$. But these solutions are unbounded when $\tau \rightarrow \infty$, so that the case (4.2) is excluded in the example of a single node in chapter 3 .

\section{Stability results for the solution of two di- mensional problems}

In this section we study the solution of a two dimensional friction problem. We use a theorem on the stability of the solution of least squares problems 
with inequality constraints proved by J. W. Daniel in 1973 [7], to obtain a stability result with respect to the applied force.

Let us assume the initial displacement $\mathbf{u}^{\text {ref }}$ is in frictional contact with the obstacle so that the equilibrium and contact conditions are fulfilled, that is

$$
\begin{cases}\mathbf{K} \mathbf{u}^{\mathrm{ref}}=\mathbf{f}^{\mathrm{ref}}+\mathbf{r}^{\mathrm{ref}} & \\ \mathbf{r}_{i}^{\mathrm{ref}}=\mathbf{0} & \text { when } i \notin I_{c} \\ u_{i, N}^{\mathrm{ref}} \leq 0, r_{i, N}^{\mathrm{ref}} \leq 0, u_{i, N}^{\mathrm{ref}} r_{i, N}^{\mathrm{ref}}=0 & \text { when } i \in I_{c} \\ \left|\mathbf{r}_{i, T}^{\mathrm{ref}}\right| \leq-\mu_{i} r_{i, N}^{\text {ref }}, & \end{cases}
$$

then the following Lemma holds.

4.3. Lemma. Let $\mathbf{f}^{n}$ be a sequence of forces such that $\mathbf{f}^{n} \rightarrow \mathbf{f}^{\text {ref }}$, and assume these forces satisfies the compatibility condition of Theorem 2.3. Let us denote the solution of the friction problem (1.5) with $\mathbf{f}^{n}$ as applied force $\left(\mathbf{u}^{n}, \mathbf{r}^{n}\right)$. Then $\left(\mathbf{u}^{n}, \mathbf{r}^{n}\right)$ is a bounded sequence.

Proof. The boundedness of $\mathbf{r}^{n}$ follows from Lemma 4.1. To prove the boundedness of $\mathbf{u}^{n}$, define $\mathbf{w}^{n}$ as in Lemma 2.2, and also assume convergence $\mathbf{w}^{n} \rightarrow \mathbf{w}$ as $\mathbf{w}^{n}$ are bounded, then

$$
\left|\mathbf{u}^{n}-\mathbf{u}_{T}^{\mathrm{ref}}\right| \mathbf{K} \mathbf{w}^{n}=\mathbf{f}^{\mathrm{ref}}+\mathbf{r}^{n}-\mathbf{K} \mathbf{u}_{T}^{\mathrm{ref}} .
$$

Since the right hand side is bounded and $\left|\mathbf{u}^{n}-\mathbf{u}_{T}^{\text {ref }}\right| \rightarrow \infty$ we see that $\mathrm{Kw}=0$.

To prove the correspondence between $\mathbf{w}$ and $\mathbf{r}$ stated in the conditions of Theorem 2.3 one only proceeds as in the proof of Theorem 2.3.

We are now able to prove:

4.4. Theorem. If $\mathbf{f}^{n}$ is a sequence of forces such that $\mathbf{f}^{n} \rightarrow \mathbf{f}^{\text {ref }}$, and if these forces satisfies the compatibility condition of Theorem 2.3, then either

CASE 1:

$$
\left\|\mathbf{u}^{n}-\mathbf{u}^{\text {ref }}\right\| \leq C_{6}\left\|\mathbf{f}^{n}-\mathbf{f}^{\text {ref }}\right\|
$$

for some constant $C_{6}$ and where $\mathbf{u}^{n}$ is a solution of the friction problem (1.5) with $\mathbf{f}^{n}$ as applied force

or

CASE 2: there is a solution $\overline{\mathbf{v}}$ to the friction problem (1.5) with initial displacement $\mathbf{u}^{\text {ref }}$ and applied force $\mathbf{f}^{\text {ref }}$ such that $\overline{\mathbf{v}} \neq \mathbf{u}^{\text {ref }}$ 
Proof. Let $\mathbf{f}^{n}$ be a sequence of forces such that $\mathbf{f}^{n} \rightarrow \mathbf{f}^{\text {ref }}$ when $n \rightarrow \infty$, and such that there are solutions $\left(\mathbf{u}^{n}, \mathbf{r}^{n}\right)$ of the friction problems

$$
\begin{cases}\mathbf{K u}=\mathbf{f}^{n}+\mathbf{r} & \\ \mathbf{r}_{i}=\mathbf{0} & \text { when } i \notin I_{c} \\ u_{i, N} \leq 0, r_{i, N} \leq 0, u_{i, N} r_{i, N}=0 & \text { when } i \in I_{c} \\ \left|\mathbf{r}_{i, T}\right| \leq-\mu_{i} r_{i, N} & \\ \mathbf{u}_{i, T}-\mathbf{u}_{i, T}^{\text {ref }}=0 & \text { if }\left|\mathbf{r}_{i, T}\right|<-\mu_{i} r_{i, N} \\ \mathbf{u}_{i, T}-\mathbf{u}_{i, T}^{\text {ref }}=-\lambda_{i} \mathbf{r}_{i, T} & \text { if }\left|\mathbf{r}_{i, T}\right|=-\mu_{i} r_{i, N}>0\end{cases}
$$

where $\lambda_{i} \geq 0$.

Lemma 4.3 implies that $\left(\mathbf{u}^{n}, \mathbf{r}^{n}\right)$ is a bounded sequence, so let us thus assume convergence $\left(\mathbf{u}^{n}, \mathbf{r}^{n}\right) \rightarrow\left(\mathbf{u}^{*}, \mathbf{r}^{*}\right)$. Assume also that CASE 1 is not valid for this sequence. By considering a subsequence, we can assume

$$
\left\|\mathbf{u}^{n}-\mathbf{u}^{\mathrm{ref}}\right\| \geq n\left\|\mathbf{f}^{n}-\mathbf{f}^{\mathrm{ref}}\right\|
$$

In two dimensions every node must be in at least one of the following states:

1. Free: $u_{i, N} \geq 0$ and $\mathbf{r}_{i}=0$.

2. Stick: $u_{i, N}=0$ and $\mathbf{u}_{i, T}-\mathbf{u}_{i, T}^{\mathrm{ref}}=0$

3. Forward sliding: $u_{i, N}=0, \mathbf{u}_{i, T}-\mathbf{u}_{i, T}^{\text {ref }} \geq 0$ and $\mathbf{r}_{i, T} \leq 0$

4. Backward sliding: $u_{i, N}=0, \mathbf{u}_{i, T}-\mathbf{u}_{i, T}^{\text {ref }} \leq 0$ and $\mathbf{r}_{i, T} \geq 0$

and thus by considering a subsequence we can assume that node $i$ always has the same state in the sequence $\left(\mathbf{u}^{n}, \mathbf{r}^{n}\right)$. Denote the indices of the nodes in of first kind $I_{\text {free }}$, the second kind $I_{\text {stick }}$ etcetera. Let $I_{C}$ denote the index set of all contact nodes.

We can now apply a theorem on the stability of the solution of least squares problems with inequality constraints proved by J. W. Daniel in 1973 [7]. This theorem implies that the solutions of

$$
\begin{aligned}
& \min _{\mathbf{v}}\left\|\mathbf{v}-\mathbf{u}^{*}\right\|^{2} \\
& \text { subject to : } \\
& \left\{\begin{array}{l}
\mathbf{K} \mathbf{v}-\mathbf{r}=\mathbf{f}^{n} \\
r_{i, N} \leq 0, \mathbf{r}_{i, T}+\mu_{i} r_{i, N} \leq 0 \text { and }-\mathbf{r}_{i, T}+\mu_{i} r_{i, N} \leq 0 \\
u_{i, N} \leq 0, r_{i, N}=0 \text { and } \mathbf{r}_{i, T}=0 \\
u_{i, N}=0 \text { and } \mathbf{u}_{i, T}-\mathbf{u}_{i, T}^{\text {ref }}=0 \\
u_{i, N}=0, \mathbf{u}_{i, T}-\mathbf{u}_{i, T}^{\text {ref }} \geq 0 \text { and } \mathbf{r}_{i, T} \leq 0 \\
u_{i, N}=0, \mathbf{u}_{i, T}-\mathbf{u}_{i, T}^{\text {ref }} \leq 0 \text { and } \mathbf{r}_{i, T} \geq 0
\end{array}\right.
\end{aligned}
$$

when $i \in I_{C}$ when $i \in I_{\text {free }}$ when $i \in I_{\text {stick }}$ when $i \in I_{\text {forward }}$ when $i \in I_{\text {backward }}$ 
satisfies

$$
\left\|\mathbf{u}^{n}-\mathbf{u}^{*}\right\| \leq C_{6}\left\|\mathbf{f}^{n}-\mathbf{f}^{\mathrm{ref}}\right\|
$$

for some constant $C_{6}$. Now, if $\mathbf{u}^{*}=\mathbf{u}^{\text {ref }}$ then CASE 1 is valid which contradicts (4.5), so that CASE 2 holds with $\overline{\mathbf{v}}=\mathbf{u}^{*} \neq \mathbf{u}^{\text {ref }}$.

\section{Example on the stability results}

Let us consider an application of Theorem 4.4. We study the solution of a two dimensional friction problem where the stiffness matrix $\mathbf{K}$ is positive definite and the initial displacement $\mathbf{u}^{\text {ref }}$ is a solution to a minimum potential energy problem,

$$
\begin{aligned}
& \min _{\mathbf{v}}\langle\mathbf{v}, \mathbf{K} \mathbf{v}\rangle-2\langle\mathbf{f}, \mathbf{v}\rangle \\
& \text { subject to : } \\
& \begin{cases}\mathbf{K v}=\mathbf{r}+\mathbf{f} & \text { for all } i \notin I_{c} \\
\mathbf{r}_{i}=\mathbf{0} & \text { for all } i \in I_{c} \\
r_{i, N} \leq 0, v_{i, N} \leq 0, r_{i, N} v_{i, N}=0 \\
\left|\mathbf{r}_{i, T}\right| \leq-\mu_{i} r_{i, N}\end{cases}
\end{aligned}
$$

The solution of (4.6) has been studied in for example Hilding [19]. It is not necessarily unique. Hilding proves that $\mathbf{r}_{i, T}^{\text {ref }}=0$, where $\mathbf{r}^{\text {ref }}=\mathbf{K} \mathbf{u}^{\text {ref }}-\mathbf{f}$.

Let us prove the following (also proved in [1]):

4.5. Lemma. If $\mathbf{u}^{\mathrm{ref}}$ is a solution of (4.6) and $(\mathbf{u}, \mathbf{r})$ is a solution of the incremental friction problem with $\mathbf{u}^{\text {ref }}$ a initial displacement and $\mathbf{f}$ as applied force, then $\mathbf{u}=\mathbf{u}^{\mathrm{ref}}$.

Proof. We have

$$
\left\langle\mathbf{u}-\mathbf{u}^{\text {ref }}, \mathbf{r}-\mathbf{r}^{\text {ref }}\right\rangle=\left\langle\mathbf{u}-\mathbf{u}^{\text {ref }}, \mathbf{K}\left(\mathbf{u}-\mathbf{u}^{\text {ref }}\right)\right\rangle \geq 0
$$

and

$$
\begin{aligned}
\left\langle\mathbf{u}-\mathbf{u}^{\text {ref }}, \mathbf{r}+\mathbf{r}^{\text {ref }}\right\rangle & =\left\langle\mathbf{u}-\mathbf{u}^{\text {ref }},-2 \mathbf{f}+\mathbf{K} \mathbf{u}+\mathbf{K} \mathbf{u}^{\text {ref }}\right\rangle \\
& =\langle\mathbf{u}, \mathbf{K u}\rangle-2\langle\mathbf{f}, \mathbf{u}\rangle-\left\langle\mathbf{u}^{\text {ref }}, \mathbf{K} \mathbf{u}^{\text {ref }}\right\rangle+2\left\langle\mathbf{f}, \mathbf{u}^{\text {ref }}\right\rangle \\
& \geq 0
\end{aligned}
$$

since $\mathbf{u}^{\text {ref }}$ is a solution of (4.6) and where $\mathbf{r}^{\text {ref }}=\mathbf{K} \mathbf{u}^{\text {ref }}-\mathbf{f}$. Using $\mathbf{r}_{i}\left(\mathbf{u}_{i}\right.$ $\left.\mathbf{u}_{i}^{\text {ref }}\right)=-\mu_{i}\left|r_{i, N}\right|\left|\mathbf{u}_{i, T}-\mathbf{u}_{i, T}^{\text {ref }}\right|-r_{i, N} u_{i, N}^{\text {ref }} \leq 0$, we find by adding (4.7) and (4.8) that

$$
0 \geq 2\left\langle\mathbf{r}, \mathbf{u}-\mathbf{u}^{\text {ref }}\right\rangle \geq\left\langle\mathbf{u}-\mathbf{u}^{\text {ref }}, \mathbf{K}\left(\mathbf{u}-\mathbf{u}^{\text {ref }}\right)\right\rangle \geq 0
$$

which yields $\mathbf{u}=\mathbf{u}^{\text {ref }}$, since $\mathbf{K}$ is assumed to be positive definite.

Thus, applying Lemma 4.5 we conclude that CASE 2 in Theorem 4.4 is impossible for a friction problem with an initial displacement which satisfies a minimum potential energy condition, so CASE 1 in Theorem 4.4 must hold for that friction problem. 


\section{Part III}

\section{Continuous Systems with Nonlocal Friction Laws}





\section{Chapter 5}

\section{Preliminaries}

This chapter formulates a friction problem for continuous bodies with a nonlocal friction law. We derive a variational inequality.

\section{Notation and problem formulation}

Let us consider an elastic body, which in its nondeformed state occupies a bounded domain $\Omega$ in $\mathbb{R}^{3}$ with a Lipschitz boundary $\Gamma$. The body $\Omega$ is subject to volume forces $\mathbf{f}$ in the domain and surface tractions $\mathbf{t}^{\text {tot }}$ on the boundary. A part $\Gamma_{C}$ has possible contact with an obstacle. Figure 5.1 illustrates the partition of the boundary $\Gamma$.

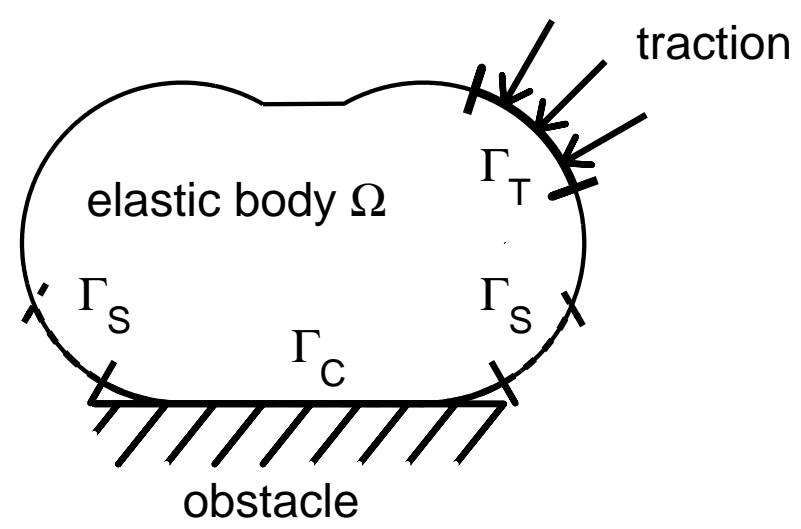

Figure 5.1: The partition of the boundary $\Gamma$. 
$\Gamma$ : $\quad$ The boundary of $\Omega$. Assumed to be Lipschitz.

$\Gamma_{T}: \quad$ Part of $\Gamma$ where nonzero tractions (that do not origin from the contact) are applied on the body.

$\Gamma_{C}$ : Part of $\Gamma$ where the body has possible contact. Assumed to be of $C^{2}$-regularity, so that the normal is continuous. The relative boundary of $\Gamma_{C}$ on $\Gamma$ is assumed to be Lipschitz.

$\Gamma_{S}: \quad$ All points on the boundary $\Gamma$ with a distance less than $\rho_{\max }$ to the contact surface $\Gamma_{C}$ for some constant $\rho_{\max }>0$. Assumed to have $C^{2}$-regularity as $\Gamma_{C}$.

We assume $\Gamma_{C}$ and $\Gamma_{T}$ are relatively open with respect to $\Gamma$, and let $\bar{\Gamma}_{C}$, $\bar{\Gamma}_{S}$ and $\bar{\Gamma}_{T}$ denote the closures of $\Gamma_{C}, \Gamma_{S}$ and $\Gamma_{T}$. We also assume that it is possible to choose $\rho_{\max }$ so that $\bar{\Gamma}_{T}$ and $\bar{\Gamma}_{S}$ are disjoint. Let $\mathbf{n}$ be a normal unit vector on $\Gamma$, directed outwards from $\Omega$, in particular $\mathbf{n}$ is continuous on $\Gamma_{S}$.

To study the existence of solutions to the friction problem we require that the shape of the contact surface $\Gamma_{C}$ is such that

$$
\left.\begin{array}{c}
\mathbf{v}(\mathbf{x}) \in \mathcal{N} \\
v_{N}(\mathbf{x}) \leq 0 \text { when } \mathbf{x} \in \Gamma_{C}
\end{array}\right\} \Rightarrow \begin{gathered}
\mathbf{v}(\mathbf{x})=\mathbf{0} \text { at most at } \\
\text { one point } \mathbf{x} \text { of } \bar{\Gamma}_{C}
\end{gathered}
$$

where $\mathbf{v}$ is a vector field and $v_{N}=\mathbf{v} \cdot \mathbf{n}$ its normal component on $\Gamma_{C}$. The set $\mathcal{N}$ is defined by $\mathcal{N}=\left\{\mathbf{v}: \mathbf{v}=\mathbf{T}+\mathbf{x} \times \mathbf{w}\right.$ where $\left.\mathbf{T}, \mathbf{w} \in \mathbb{R}^{3}\right\}$, where $\mathbf{x}$ is the radius vector with respect to some origin and $\times$ is the cross product. Geometrically the requirement (5.1) means that if $\mathbf{v}$ is a rotation satisfying $v_{N} \leq 0$ on $\Gamma_{C}$, then the axis of rotation is only allowed to intersect the contact surface $\Gamma_{C}$ once. Figure 5.2 illustrates a problem which satisfies (5.1), and Figure 5.3 illustrates a problem which does not.

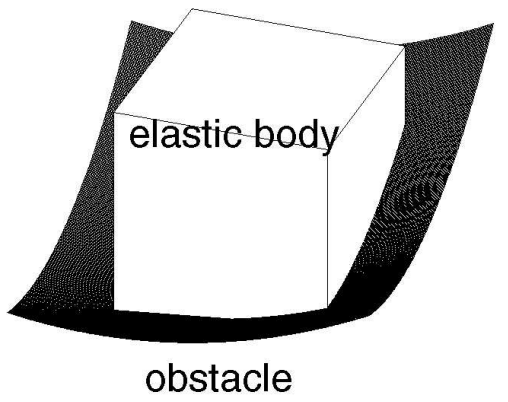

Figure 5.2: A friction problem which satisfies (5.1).

Denote the components of the stress tensor $\boldsymbol{\sigma}$ by $\sigma_{i j}$ where the indices $i, j \in\{1,2,3\}$ refer to different coordinate directions in an orthogonal 


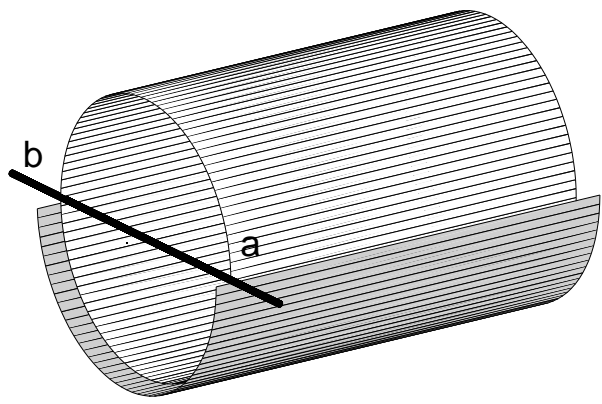

Figure 5.3: A friction problem which violates (5.1). The cylinder can rotate about the axis $a b$, which intersects the contact surface twice.

coordinate frame. The stress tensor $\boldsymbol{\sigma}$ obeys the equation

$$
\left\{\begin{array}{c}
\operatorname{div}(\boldsymbol{\sigma})+\mathbf{f}=\mathbf{0} \\
\boldsymbol{\sigma} \cdot \mathbf{n}=\mathbf{t}^{t o t} \text { on } \Gamma
\end{array}\right.
$$

which expresses the equilibrium of force.

The field of displacements is denoted by $\mathbf{u}$. The displacement field $\mathbf{u}$ causes strains $\boldsymbol{\epsilon}$ inside the body. The components of the linearized strains are

$$
\epsilon_{i j}=\frac{1}{2}\left(\frac{\partial u_{i}}{\partial x_{j}}+\frac{\partial u_{j}}{\partial x_{i}}\right)
$$

where $i, j \in\{1,2,3\}$ and $x_{i}$ is the $i$ :th coordinate. No displacements $\mathbf{u}$ are prescribed.

The assumption on linear elasticity implies $\boldsymbol{\sigma}=\mathbb{E} \boldsymbol{\epsilon}$, where $\mathbb{E}$ is a tensor of order 4 , so that

$$
\sigma_{i j}=\sum_{k, l \in\{1,2,3\}} \mathbb{E}_{i j k l} \epsilon_{k l}
$$

where $i, j \in\{1,2,3\}$. The components $\mathbb{E}_{i j k l}$ of $\mathbb{E}$ fulfill the symmetry conditions $\mathbb{E}_{i j k l}=\mathbb{E}_{j i k l}=\mathbb{E}_{k l i j}$ for all $i, j, k, l \in\{1,2,3\}$. We assume $\mathbb{E}_{i j k l} \in L^{\infty}(\Omega)$ and that these components satisfy the an ellipticity condition

$$
\sum_{i, j, k, l \in\{1,2,3\}} \mathbb{E}_{i j k l} \epsilon_{i j} \epsilon_{k l} \geq C_{7} \sum_{k, l \in\{1,2,3\}} \epsilon_{k l} \epsilon_{k l}
$$

for some constant $C_{7}$. 
The body has possible contact with an obstacle on $\Gamma_{C}$, and this contact causes contact forces $\mathbf{p}$. Let us rewrite $(5.2)$ as $\boldsymbol{\sigma} \cdot \mathbf{n}=\mathbf{t}+\mathbf{p}$ where $\mathbf{t}=\mathbf{t}^{t o t}-\mathbf{p}$ is the traction not caused by the contact. The support of $\mathbf{t}$ is assumed to be within $\Gamma_{T}$.

The normal and tangential component of $\mathbf{u}$ and $\mathbf{p}$ are

$$
\begin{array}{ll}
p_{N}=\mathbf{n} \cdot \mathbf{p}, & \mathbf{p}_{T}=\mathbf{p}-p_{N} \mathbf{n}, \\
u_{N}=\mathbf{n} \cdot \mathbf{u}, & \mathbf{u}_{T}=\mathbf{u}-u_{N} \mathbf{n} .
\end{array}
$$

The contact force $\mathbf{p}$ is defined by a law of contact and a law of friction. We use the contact law of Signorini, which reads

$$
p_{N} \leq 0, \quad u_{N}-g \leq 0 \quad \text { and } \quad p_{N}\left(u_{N}-g\right)=0 \text { on } \Gamma_{C}
$$

where $g \in H^{1 / 2}(\Gamma)$ is the distance from the obstacle to the boundary of the body in its nondeformed state. We also assume that $g$ is greater than zero and bounded, $0 \leq g \leq C_{8}<\infty$ everywhere on $\bar{\Gamma}_{C}$ for some constant $C_{8}$. Moreover, we use an incremental nonlocal law of friction which requires $\left|\mathbf{p}_{T}\right| \leq-\mu S\left(p_{N}\right)$ and

$$
\left\{\begin{array}{l}
\left|\mathbf{p}_{T}\right|<-\mu S\left(p_{N}\right) \Rightarrow \mathbf{u}_{T}-\mathbf{u}_{T}^{\text {ref }}=\mathbf{0} \\
\left|\mathbf{p}_{T}\right|=-\mu S\left(p_{N}\right) \Rightarrow \exists \lambda \geq 0, \mathbf{u}_{T}-\mathbf{u}_{T}^{\text {ref }}=-\lambda \mathbf{p}_{T}
\end{array}\right.
$$

on $\Gamma_{C}$, where $\mathbf{u}^{\text {ref }}$ is a previous displacement field which is assumed to be in equilibrium and satisfy the contact constraints, $\mu \in L^{\infty}(\Gamma)$ is the coefficient of friction which satisfies $\mu_{\max } \geq \mu \geq \mu_{\min }>0$ almost everywhere for some constants $\mu_{\max }$ and $\mu_{\min }$ and $S$ is a linear and compact mapping $H^{-1 / 2}(\Gamma) \rightarrow L^{2}(\Gamma)$. We will focus on a particular choice of the mapping $S$. To define this mapping, let $Q$ be

$$
Q(t)= \begin{cases}e^{\frac{1}{t^{2}-1}} & \text { if }|t|<1 \\ 0 & \text { otherwise. }\end{cases}
$$

and define $\omega$ by

$$
\begin{aligned}
\omega_{\mathbf{x}}(\mathbf{y}) & =\omega(|\mathbf{x}-\mathbf{y}| / \rho)=C_{\omega} Q(|\mathbf{x}-\mathbf{y}| / \rho) \\
& = \begin{cases}C_{\omega} e^{\frac{\rho^{2}}{|\mathbf{x}-\mathbf{y}|^{2}-\rho^{2}}} & \text { if }|\mathbf{x}-\mathbf{y}|<\rho \\
0 & \text { otherwise }\end{cases}
\end{aligned}
$$

where $|\mathbf{x}-\mathbf{y}|$ is the distance between the two points $\mathbf{x} \in \Gamma$ and $\mathbf{y} \in \Gamma$ and $\rho$ and $C_{\omega}$ are constants greater than zero. We choose $\rho$ so that $\rho<\rho_{\max }$. Using the definition of $\omega$ in (5.6), let $S$ be defined by

$$
S(\cdot)(\mathbf{x})=\left[\omega_{\mathbf{x}}, \cdot\right]_{\bar{\Gamma}_{C}}
$$


where $\mathbf{x} \in \Gamma, \bar{\Gamma}_{C}$ is the closure of $\Gamma_{C}$ in $\Gamma$ and $[\chi, \psi]_{\bar{\Gamma}_{C}}$ is the duality pairing of $\chi \in H^{1 / 2}\left(\bar{\Gamma}_{C}\right)$ and $\psi \in H^{-1 / 2}\left(\bar{\Gamma}_{C}\right)$. This definition is similar to the definition of Kikuchi et. al. in [22].

In the following propositions it will be convenient to let $C_{\omega}=\frac{C^{*}}{\rho^{2}}$, where $C^{*}$ is independent on $\rho$, so we make this assumption from now on.

5.1. Lemma. Let

$$
\omega_{\mathbf{x}}(\mathbf{y})=C_{\omega} Q(|\mathbf{x}-\mathbf{y}| / \rho)= \begin{cases}C_{\omega} e^{\frac{\rho^{2}}{|\mathbf{x}-\mathbf{y}|^{2}-\rho^{2}}} & \text { if }|\mathbf{x}-\mathbf{y}|<\rho \\ 0 & \text { otherwise }\end{cases}
$$

where $|\mathbf{x}-\mathbf{y}|$ is the distance between the two points $\mathbf{x} \in \Gamma_{S}$ and $\mathbf{y} \in \Gamma_{S}$ and $\rho$ and $C_{\omega}$ are constants greater than zero. If $C_{\omega}=C^{*} / \rho^{2}$ where $C^{*}$ is a constant, then it is possible to choose this constant $C^{*}$ so that

$$
\left[\omega_{\overline{\mathbf{x}}}, 1\right]_{\Gamma_{S}}=\int_{\Gamma_{S}} \omega_{\overline{\mathbf{x}}}(\mathbf{y}) \mathrm{d} \Gamma_{\mathbf{y}} \rightarrow 1 \text { when } \rho \rightarrow 0
$$

uniformly with respect to $\overline{\mathbf{x}} \in \Gamma_{C}$.

5.2. Lemma. Let $h: \mathbb{R}^{3} \rightarrow \mathbb{R}$ denote a Lipschitz function and $\Gamma^{\prime} \subset \Gamma_{C}$. Then, there exist a bounded function, $\Phi(\overline{\mathbf{x}}, \rho)$, such that $\Phi(\overline{\mathbf{x}}, \rho)<\Phi_{\max }<$ $\infty$ for all $\overline{\mathbf{x}} \in \bar{\Gamma}_{C}$, and a constant $C_{9}$ which only depends on the Lipschitz constant of $h$, such that

$$
\left|\left\langle\omega_{\overline{\mathbf{x}}}, h\right\rangle_{\Gamma^{\prime}}-\Phi(\overline{\mathbf{x}}, \rho) h(\overline{\mathbf{x}})\right| \leq C_{9} \rho .
$$

for all $\overline{\mathbf{x}} \in \bar{\Gamma}_{C}$.

The notation $\langle\cdot, \cdot\rangle_{\Gamma^{\prime}}$ refers to the inner product on $L^{2}\left(\Gamma^{\prime}\right)$, that is

$$
\langle f, g\rangle_{\Gamma^{\prime}}=\int_{\Gamma^{\prime}} f g \mathrm{~d} \Gamma
$$

where $\mathrm{d} \Gamma$ is a measure of surface area on $\Gamma$. Lemma 5.1 and Lemma 5.2 are proved in Chapter 6 section 3 .

\section{Variational formulation}

Let us define the bilinear elastic energy functional

$$
a(\mathbf{u}, \mathbf{v})=\int_{\Omega} \sum_{i, j \in\{1,2,3\}} \epsilon(\mathbf{u})_{i j} \sigma(\mathbf{v})_{i j} \mathrm{~d} \Omega
$$

and let us use the inner products

$$
\begin{aligned}
& \langle f, g\rangle_{\Omega}=\int_{\Omega} f \cdot g \mathrm{~d} \Omega \\
& \langle f, g\rangle_{\Gamma}=\int_{\Gamma} f \cdot g \mathrm{~d} \Gamma
\end{aligned}
$$


where $d \Omega$ is the usual measure of volume and $d \Gamma$ is a measure of surface area on the boundary. We denote the corresponding norms $\|\cdot\|_{L^{2}(\Omega)}$ and $\|\cdot\|_{L^{2}(\Gamma)}$. We also use the Sobolev norm $\|\cdot\|_{H^{1}(\Omega)}$, the supremum norm $\|\cdot\|_{L^{\infty}(\Gamma)}$ and write the duality pairing of $\chi \in H^{1 / 2}(\Gamma)$ and $\psi \in H^{-1 / 2}(\Gamma)$ as $[\chi, \psi]_{\Gamma}$. To assign boundary values, we use a trace mapping (see [22] or $[3]), H^{1}(\Omega) \rightarrow H^{1 / 2}(\Gamma)$, such that

$$
\begin{aligned}
& \text { - } u^{n} \rightarrow u \text { weakly in } H^{1}(\Omega) \\
& \quad \Rightarrow u^{n} \rightarrow u \text { strongly in } L^{2}(\Gamma) \\
& \text { - }\|u\|_{L^{2}(\Gamma)} \leq C_{10}\|u\|_{H^{1}(\Omega)}
\end{aligned}
$$

for some constant $C_{10}$.

From the properties of the tensor $\mathbb{E}$ in (5.3) follows that the bilinear elastic energy functional $a(\cdot, \cdot)$ is symmetric, i.e. $a(\mathbf{u}, \mathbf{v})=a(\mathbf{v}, \mathbf{u})$ for all $\mathbf{u} \in H^{1}(\Omega)$ and $\mathbf{v} \in H^{1}(\Omega)$, and also that $a(\cdot, \cdot)$ is semicoercive, so that $a(\mathbf{u}, \mathbf{u}) \geq 0$. Moreover,

$$
a(\mathbf{u}, \mathbf{v}) \leq C_{11}\|\mathbf{u}\|_{H^{1}(\Omega)}\|\mathbf{v}\|_{H^{1}(\Omega)}
$$

for some constant $C_{11}$, and since no displacements are prescribed

$$
\begin{aligned}
\mathcal{N} & =\left\{\mathbf{v}: \mathbf{v}=\mathbf{T}+\mathbf{x} \times \mathbf{w} \text { where } \mathbf{T}, \mathbf{w} \in \mathbb{R}^{3}\right\} \\
& =\left\{\mathbf{v} \in H^{1}(\Omega): a\left(\mathbf{v}, \mathbf{v}^{\prime}\right)=0 \text { for every } \mathbf{v}^{\prime} \in H^{1}(\Omega)\right\}
\end{aligned}
$$

where $\mathbf{x}$ is the radius vector with respect to some origin and $x$ is the cross product. The set $\mathcal{N}$ is called the set of rigid body displacements. The orthogonal projection map onto $\mathcal{N}$ in $L^{2}(\Omega)$ is denoted by $\mathbf{P}$. We have

$$
a(\mathbf{v}, \mathbf{v}) \geq C_{12}\|\mathbf{v}-\mathbf{P} \mathbf{v}\|_{H^{1}(\Omega)}^{2}
$$

for all $\mathbf{v} \in H^{1}(\Omega)$ and where $C_{12}>0$. This is proved in [11], and also used in for example [6]. Moreover, the set of rigid body motions $\mathcal{N}$ is finite dimensional, and thus the $H^{1}$-norm and the $L^{2}$-norm are equivalent on $\mathcal{N}$, that is

$$
C_{13}\|\mathbf{v}\|_{H^{1}(\Omega)} \leq\|\mathbf{v}\|_{L^{2}(\Omega)}
$$

for all $\mathbf{v} \in \mathcal{N}$, where $C_{13}$ is a constant greater than zero.

Let us assume $\mathbf{f} \in L^{2}(\Omega)$ and $\mathbf{t} \in L^{2}\left(\Gamma_{T}\right)$. The incremental friction problem defined by (5.2), (5.3), (5.4) and (5.5) can be formulated as a variational problem, see [22], which is to find $\mathbf{u} \in \mathbb{V}=\left\{\mathbf{v} \in H^{1}(\Omega): v_{N} \leq\right.$ $g$ on $\left.\Gamma_{C}\right\}$ such that

$$
\begin{aligned}
(L, \mathbf{v}-\mathbf{u}) \leq & a(\mathbf{u}, \mathbf{v}-\mathbf{u}) \\
& +\left\langle\mu S\left(p_{N}\right),\left|\mathbf{u}_{T}-\mathbf{u}_{T}^{\mathrm{ref}}\right|-\left|\mathbf{v}_{T}-\mathbf{u}_{T}^{\mathrm{ref}}\right|\right\rangle_{\Gamma_{S}}
\end{aligned}
$$


for all $\mathbf{v} \in \mathbb{V}$, where

$$
\begin{aligned}
(L, \mathbf{v}) & =\int_{\Omega} \mathbf{f} \cdot \mathbf{v} \mathrm{d} \Omega+\int_{\Gamma_{T}} \mathbf{t} \cdot \mathbf{v} \mathrm{d} \Gamma \\
a(\mathbf{u}, \mathbf{v}) & =\int_{\Omega} \sum_{i, j \in\{1,2,3\}} \boldsymbol{\epsilon}(\mathbf{u})_{i j} \boldsymbol{\sigma}(\mathbf{v})_{i j} \mathrm{~d} \Omega
\end{aligned}
$$

and $\int_{\Omega} \cdot \mathrm{d} \Omega$ is an integral of volume and $\int_{\Gamma} \cdot \mathrm{d} \Gamma$ is an integral on the boundary with respect to a measure of surface $\mathrm{d} \Gamma$. The initial state $\mathbf{u}^{\text {ref }} \in \mathbb{V}=$ $\left\{\mathbf{v} \in H^{1}(\Omega): v_{N} \leq g\right.$ on $\left.\Gamma_{C}\right\}$ is assumed to fulfill the contact law (5.4).

In proving the next lemma we use Green's formula

$$
a(\mathbf{u}, \mathbf{v})+\langle\operatorname{div}(\boldsymbol{\sigma}), \mathbf{v}\rangle_{\Omega}=[\mathbf{v}, \boldsymbol{\sigma} \cdot \mathbf{n}]_{\Gamma}
$$

where $\mathbf{v} \in H^{1}(\Omega)$. The content of the next lemma is that we recover the contact law (5.4) and the law of friction (5.5) from the variational inequality (5.13).

5.3. Lemma. If $\mathbf{u}$ satisfies

$$
\begin{aligned}
(L, \mathbf{v}-\mathbf{u}) \leq & a(\mathbf{u}, \mathbf{v}-\mathbf{u}) \\
& +\left\langle\mu S\left(p_{N}\right),\left|\mathbf{u}_{T}-\mathbf{u}_{T}^{\mathrm{ref}}\right|-\left|\mathbf{v}_{T}-\mathbf{u}_{T}^{\mathrm{ref}}\right|\right\rangle_{\Gamma_{S}}
\end{aligned}
$$

for all $\mathbf{v} \in \mathbb{V}=\left\{\mathbf{v} \in H^{1}(\Omega): v_{N} \leq g\right.$ on $\left.\Gamma_{C}\right\}$, then $\operatorname{div}(\boldsymbol{\sigma})+\mathbf{f}=\mathbf{0}$ in $\Omega$, $\boldsymbol{\sigma} \cdot \mathbf{n}=\mathbf{t}$ on $\Gamma_{T}$ and

$$
[\mathbf{v}-\mathbf{u}, \mathbf{p}] \geq-\left\langle\mu S\left(p_{N}\right),\left|\mathbf{u}_{T}-\mathbf{u}_{T}^{\mathrm{ref}}\right|-\left|\mathbf{v}_{T}-\mathbf{u}_{T}^{\mathrm{ref}}\right|\right\rangle_{\Gamma_{S}}
$$

for all $\mathbf{v} \in \mathbb{V}$ and where the support of $p_{N}$ is in $\bar{\Gamma}_{C}$. From (5.16) follows

$$
\begin{array}{ll}
p_{N} \leq 0 & \text { on } \Gamma_{C} \\
\left|\mathbf{p}_{T}\right| \leq-\mu S\left(p_{N}\right) & \text { on } \Gamma_{S}
\end{array}
$$

and if $\left|\mathbf{p}_{T}\right|=-\mu S\left(p_{N}\right)>0$ then $\mathbf{u}_{T}=-\lambda \mathbf{p}_{T}$ for some $\lambda \geq 0$. By $p_{N} \leq 0$ we mean that $-p_{N}$ is a positive measure.

Proof. Green's formula, $a(\mathbf{u}, \mathbf{v}-\mathbf{u})+\langle\operatorname{div}(\boldsymbol{\sigma}), \mathbf{v}-\mathbf{u}\rangle_{\Omega}=[\mathbf{v}-\mathbf{u}, \boldsymbol{\sigma} \cdot \mathbf{n}]_{\Gamma}$, and (5.15) imply

$$
\begin{aligned}
\langle\mathbf{f}, \mathbf{v}-\mathbf{u}\rangle_{\Omega}+\langle\mathbf{t}, \mathbf{v}-\mathbf{u}\rangle_{\Gamma_{T}} \leq & {[\mathbf{v}-\mathbf{u}, \boldsymbol{\sigma} \cdot \mathbf{n}]_{\Gamma_{S}}-\langle\operatorname{div}(\boldsymbol{\sigma}), \mathbf{v}-\mathbf{u}\rangle_{\Omega} } \\
& +\left\langle\mu S\left(p_{N}\right),\left|\mathbf{u}_{T}-\mathbf{u}_{T}^{\mathrm{ref}}\right|-\left|\mathbf{v}_{T}-\mathbf{u}_{T}^{\mathrm{ref}}\right|\right\rangle_{\Gamma_{S}}
\end{aligned}
$$

By choosing $\mathbf{v}$ so that $\mathbf{v}-\mathbf{u}=\mathbf{0}$ on $\Gamma$, we conclude $\langle-\operatorname{div}(\boldsymbol{\sigma}), \mathbf{v}\rangle_{\Omega}=\langle\mathbf{f}, \mathbf{v}\rangle_{\Omega}$ and thus $-\operatorname{div}(\boldsymbol{\sigma})=\mathbf{f}$ in the sense of distributions. We obtain

$$
\begin{aligned}
\langle\mathbf{t}, \mathbf{v}-\mathbf{u}\rangle_{\Gamma_{T}} \leq & {[\mathbf{v}-\mathbf{u}, \boldsymbol{\sigma} \cdot \mathbf{n}]_{\Gamma} } \\
& +\left\langle\mu S\left(p_{N}\right),\left|\mathbf{u}_{T}-\mathbf{u}_{T}^{\mathrm{ref}}\right|-\left|\mathbf{v}_{T}-\mathbf{u}_{T}^{\mathrm{ref}}\right|\right\rangle_{\Gamma_{S}}
\end{aligned}
$$


and if $\mathbf{v}=\mathbf{u}$ on $\Gamma_{S}$, we see that $\boldsymbol{\sigma} \cdot \mathbf{n}=\mathbf{t}$ on $\Gamma_{T}$ and $\boldsymbol{\sigma} \cdot \mathbf{n}=\mathbf{0}$ on $\Gamma \backslash\left(\bar{\Gamma}_{T} \cup \bar{\Gamma}_{S}\right)$. By $\bar{\Gamma}_{T}$ we mean the closure of $\Gamma_{T}$ and by $\bar{\Gamma}_{S}$ we mean the closure of $\Gamma_{S}$.

Let $\mathbf{v}_{T}=\mathbf{u}_{T}$ and use that $\boldsymbol{\sigma} \cdot \mathbf{n}=\mathbf{p}$ on $\Gamma_{S}$, then we find

$$
0 \leq\left[v_{N}-u_{N}, p_{N}\right]_{\Gamma_{S}}
$$

which yields $\left[u_{N}-g, p_{N}\right]_{\Gamma_{C}}=0$ if we choose $v_{N}=g$ and $v_{N}=2 u_{N}-g$. We also find $p_{N} \leq 0$ on $\Gamma_{C}$. Let $v_{N}=u_{N}$ in (5.17),

$$
0 \leq\left[\mathbf{v}_{T}-\mathbf{u}_{T}, \mathbf{p}_{T}\right]_{\Gamma_{S}}+\left\langle\mu S\left(p_{N}\right),\left|\mathbf{u}_{T}-\mathbf{u}_{T}^{\mathrm{ref}}\right|-\left|\mathbf{v}_{T}-\mathbf{u}_{T}^{\mathrm{ref}}\right|\right\rangle_{\Gamma_{S}} .
$$

This holds for all $\mathbf{v}_{T}$, which implies

$$
0 \leq\left[\mathbf{v}_{T}, \mathbf{p}_{T}\right]_{\Gamma_{S}}+\left[\left|\mathbf{v}_{T}\right|,-\mu S\left(p_{N}\right)\right]_{\Gamma_{S}}
$$

so that $\left|\mathbf{p}_{T}\right| \leq-\mu S\left(p_{N}\right)$ almost everywhere. Moreover let $\mathbf{v}_{T}=\mathbf{u}_{T}^{\text {ref }}$ in (5.18), then

$$
0 \leq\left[\mathbf{u}_{T}^{\mathrm{ref}}-\mathbf{u}_{T}, \mathbf{p}_{T}\right]_{\Gamma_{S}}+\left\langle\mu S\left(p_{N}\right),\left|\mathbf{u}_{T}-\mathbf{u}_{T}^{\mathrm{ref}}\right|\right\rangle_{\Gamma_{S}}
$$

and since $-\mu S\left(p_{N}\right) \geq\left|\mathbf{p}_{T}\right|$ we find

$$
\left\{\begin{array}{l}
\left|\mathbf{p}_{T}\right|<-\mu S\left(p_{N}\right) \Rightarrow \mathbf{u}_{T}-\mathbf{u}_{T}^{\mathrm{ref}}=\mathbf{0} \\
\left|\mathbf{p}_{T}\right|=-\mu S\left(p_{N}\right) \Rightarrow \exists \lambda \geq 0, \mathbf{u}_{T}-\mathbf{u}_{T}^{\mathrm{ref}}=-\lambda \mathbf{p}_{T}
\end{array}\right.
$$

We have $\boldsymbol{\sigma} \cdot \mathbf{n}=\mathbf{t}$ on $\Gamma_{T}, \boldsymbol{\sigma} \cdot \mathbf{n}=\mathbf{p}$ on $\Gamma_{S}$ and $\boldsymbol{\sigma} \cdot \mathbf{n}=\mathbf{0}$ on $\Gamma \backslash\left(\bar{\Gamma}_{S} \cup \bar{\Gamma}_{T}\right)$, so we can write Green's formula (5.14)

$$
a(\mathbf{u}, \mathbf{v})+\langle\operatorname{div}(\boldsymbol{\sigma}), \mathbf{v}\rangle_{\Omega}=[\mathbf{v}, \mathbf{p}]_{\Gamma_{S}}+\langle\mathbf{t}, \mathbf{v}\rangle_{\Gamma_{T}}
$$

while studying this friction problem.

The following results for the bilinear energyform $a(\cdot, \cdot)$ are used in this part of the thesis.

5.4. Lemma. I. If $\mathbf{u}^{n} \rightarrow \mathbf{u}^{*}$ by weak convergence in $H^{1}(\Omega)$, then

$$
a\left(\mathbf{u}^{*}, \mathbf{u}^{*}\right) \leq \liminf _{n \rightarrow \infty} a\left(\mathbf{u}^{n}, \mathbf{u}^{n}\right)
$$

and

$$
\left\langle\mathbf{u}^{*}, \mathbf{u}^{*}\right\rangle_{\Omega}=\liminf _{n \rightarrow \infty}\left\langle\mathbf{u}^{n}, \mathbf{u}^{n}\right\rangle_{\Omega}
$$

II. Let $\varphi \in L^{\infty}(\Omega)$ be a function $\Omega \rightarrow \mathbb{R}$ bounded from below by a positive constant $\varphi_{\min }$ so that $\varphi \geq \varphi_{\text {min }}>0$. Then there exists a constant $C_{15}>0$ such that

$$
\langle\varphi \mathbf{u}, \mathbf{u}\rangle_{\Omega}+a(\mathbf{u}, \mathbf{u}) \geq C_{15}\|\mathbf{u}\|_{H^{1}(\Omega)}^{2}
$$

for all $\mathbf{u} \in H^{1}(\Omega)$ 
Proof. $\quad$ I. (5.20) follows from

$$
\begin{aligned}
0 & \leq \liminf _{n \rightarrow \infty} a\left(\mathbf{u}^{*}-\mathbf{u}^{n}, \mathbf{u}^{*}-\mathbf{u}^{n}\right) \\
& =\liminf _{n \rightarrow \infty}\left[a\left(\mathbf{u}^{*}, \mathbf{u}^{*}\right)-2 a\left(\mathbf{u}^{*}, \mathbf{u}^{n}\right)+a\left(\mathbf{u}^{n}, \mathbf{u}^{n}\right)\right] \\
& =-a\left(\mathbf{u}^{*}, \mathbf{u}^{*}\right)+\liminf _{n \rightarrow \infty} a\left(\mathbf{u}^{n}, \mathbf{u}^{n}\right)
\end{aligned}
$$

and $\mathbf{u}^{n} \rightarrow \mathbf{u}^{*}$ by weak convergence in $H^{1}(\Omega)$ means that $\mathbf{u}^{n} \rightarrow \mathbf{u}^{*}$ in $L^{2}(\Omega)$, so that (5.21) follows.

II. Assume (5.22) is false, then we can construct a sequence $\left\{\mathbf{v}^{n}\right\}_{n=1}^{\infty}$ such that $\left\|\mathbf{v}^{n}\right\|_{H^{1}(\Omega)}=1$ and

$$
\left\langle\varphi \mathbf{v}^{n}, \mathbf{v}^{n}\right\rangle_{\Omega}+a\left(\mathbf{v}^{n}, \mathbf{v}^{n}\right) \rightarrow 0
$$

when $n \rightarrow \infty$. By Alaoglu's theorem, there exists a $\mathbf{v}^{*}$ such that $\mathbf{v}^{n} \rightarrow \mathbf{v}^{*}$ in $H^{1}(\Omega)$. Thus, (5.20) and (5.21) yield $a\left(\mathbf{v}^{*}, \mathbf{v}^{*}\right)=0$ and $\left\langle\mathbf{v}^{*}, \mathbf{v}^{*}\right\rangle_{\Omega}=0$, which implies $\mathbf{v}^{*}=\mathbf{0}$. From (5.11) we obtain $\mathbf{v}^{n}-\mathbf{P} \mathbf{v}^{n} \rightarrow \mathbf{0}$ in $H^{1}(\Omega)$, and we have $\mathbf{P v}^{n} \rightarrow \mathbf{P v}^{*}=\mathbf{0}$ since $\mathcal{N}$ is finite dimensional. Hence $\mathbf{v}^{n} \rightarrow \mathbf{v}^{*}=\mathbf{0}$ strongly in $H^{1}(\Omega)$, and thus $0=\left\|\mathbf{v}^{*}\right\|_{H^{1}(\Omega)}=\lim _{n \rightarrow \infty}\left\|\mathbf{v}^{n}\right\|_{H^{1}(\Omega)}=1$, so that we have a contradiction.

remark: Instead of the problem (5.15) it is possible to study the variational problem:

$$
\begin{aligned}
(L, \mathbf{v}-\mathbf{u}) \leq & a(\mathbf{u}, \mathbf{v}-\mathbf{u}) \\
& +\left\langle\mu S\left(p_{N}\right),\left|\mathbf{u}_{T}-\mathbf{u}_{T}^{\mathrm{ref}}\right|-\left|\mathbf{v}_{T}-\mathbf{u}_{T}^{\mathrm{ref}}\right|\right\rangle_{\Gamma_{C}}
\end{aligned}
$$

for all $\mathbf{v} \in \mathbb{V}$. This inequality implies that $\mathbf{p}_{T}=\mathbf{0}$ on $\Gamma \backslash \Gamma_{C}$, so we have no tangential contact force on $\Gamma_{S} \backslash \Gamma_{C}$ unlike (5.15). However, this means that the resultant of the tangential contact force has been reduced, and this implies that we have restricted the compatibility condition on the applied force. Compare the example in Chapter 7 section 2. 


\section{Chapter 6}

\section{Existence results for nonlocal friction laws}

This part of the thesis presents a sufficient condition for the existence of solutions to the noncoercive incremental friction problem with a nonlocal Coulomb friction law. The condition requires compatibility of the applied force and the mollifier to have a small support.

\section{Existence theorem for three dimensional problems}

The existence of solutions to the variational inequality (5.13) in the coercive case has been proved for example by Cocou [6] and by Kikuchi et. al. [22] by use of fixed point theorems. Thus, they prove the existence of a solution in a subspace $\mathcal{B}$ of $H^{1}(\Omega)$ such that

$$
a(\mathbf{v}, \mathbf{v}) \geq C_{16}\|\mathbf{v}\|_{H^{1}(\Omega)}^{2}
$$

for all $\mathbf{v} \in \mathcal{B}$. The subspace $\mathcal{B}$ can for example consist of all displacement fields such that the displacement is zero on a part of the boundary of positive measure.

Some friction problems do not fulfill a coercive condition (6.1), such friction problems are called noncoercive or semicoercive. These problems typically occur when the body is not sufficiently anchored, for example when the body lacks support. The aim of the rest of this section is to study the existence of solutions to the friction problem (5.13) when the energy functional $a(\cdot, \cdot)$ is semicoercive.

To study the noncoercive friction problem we consider the sequence of 
problems

$$
\begin{aligned}
(L, \mathbf{v}-\mathbf{u}) \leq & \varepsilon_{n}\langle\varphi \mathbf{u}, \mathbf{v}-\mathbf{u}\rangle_{\Omega}+a(\mathbf{u}, \mathbf{v}-\mathbf{u}) \\
& +\left\langle\mu S\left(p_{N}\right),\left|\mathbf{u}_{T}-\mathbf{u}_{T}^{\mathrm{ref}}\right|-\left|\mathbf{v}_{T}-\mathbf{u}_{T}^{\mathrm{ref}}\right|\right\rangle_{\Gamma_{S}}
\end{aligned}
$$

for all $\mathbf{v} \in \mathbb{V}=\left\{\mathbf{v} \in H^{1}(\Omega): v_{N} \leq g\right.$ on $\left.\Gamma_{C}\right\}$, where $\varepsilon_{n} \rightarrow 0$ as $n \rightarrow 0$ and $\varphi \in L^{\infty}(\Omega)$. If we require $\varphi \geq \varphi_{\text {min }}>0$ and $\varepsilon_{n}>0$, where $\varphi_{\text {min }}$ is a constant, then the auxiliary problem (6.2) is coercive according to the Lemma 5.4. Hence there are solutions of this problem according to the existence theorems for coercive friction problems presented by Cocou ([6], sect. 2) and Kikuchi et. al. ([22], Ch. 11.5). Thus we obtain a sequence of solutions $\left\{\mathbf{u}^{n}\right\}_{n=1}^{\infty}$ as $\varepsilon_{n} \rightarrow 0$. Let $\mathbf{p}^{n}$ be the corresponding sequence of contact forces. The contact law (5.4) and Coulomb friction law (5.5) are fulfilled, as proved in Lemma 5.3.

In this chapter we derive compatibility conditions that make it possible to construct a solution to the original friction problem (5.13) from this sequence. To do this we frequently use a special case of Alaoglu's theorem. Alaoglu's theorem implies that a bounded sequence in a Hilbert space has a weakly convergent subsequence. We focus on the limits of these convergent subsequences, so we often assume that the full sequence converges weakly to simplify the notation without loss of generality. We also use the following theorem on measures.

Let $\left\{\nu_{k}\right\}_{k=1}^{\infty}$ be a sequence of (positive) Radon measures on $\mathbb{R}^{n}$ satisfying

$$
\sup _{k} \nu_{k}(K)<\infty \text { for each compact set } K \subset \mathbb{R}^{n} .
$$

Then there exists a subsequence $\left\{\nu_{k_{j}}\right\}_{j=1}^{\infty}$ and a Radon measure $\tilde{\nu}$ such that

$$
\lim _{j \rightarrow \infty} \int f \mathrm{~d} \nu_{k_{j}}=\int f \mathrm{~d} \tilde{\nu}
$$

for any continuous $f \in \mathbb{R}^{n}$ with compact support.

This theorem on measures is proved in for example [12]. One result of the theorem above is the following lemma.

6.1. Lemma. If $\nu^{n}$ is a sequence of positive measures, such that the total variation $\left\{\left[1, \nu^{n}\right]_{\bar{\Gamma}_{C}}\right\}_{n=1}^{\infty}$ is uniformly bounded with respect to $n$, then $\left\{S\left(\nu^{n}\right)\right\}_{n=1}^{\infty}$ has a subsequence which converges strongly in $L^{2}\left(\Gamma_{S}\right)$.

Proof. To prove $L^{2}$-convergence of a subsequence of $\left\{S\left(\nu^{n}\right)\right\}$ we use Lebesgue's dominated convergence theorem, and thus need to verify pointwise convergence and uniform boundedness for some subsequence of $\left\{S\left(\nu^{n}\right)\right\}_{n=1}^{\infty}$. Since 
$\left[1, \nu^{n}\right]_{\bar{\Gamma}_{C}}$ is bounded, there is a subsequence converging weakly according to $(6.3)$, and thus we can define $\tilde{\nu}$ so that

$$
S\left(\nu^{n}\right)(\mathbf{x})=\left[\omega_{\mathbf{x}}, \nu^{n}\right]_{\bar{\Gamma}_{C}} \rightarrow\left[\omega_{\mathbf{x}}, \tilde{\nu}\right]_{\bar{\Gamma}_{C}}=S(\tilde{\nu})(\mathbf{x})
$$

for all $\mathbf{x} \in \Gamma$, which is pointwise convergence of $S\left(\nu^{n}\right)$. Furthermore $\omega_{\mathbf{x}} \leq$ $C_{\omega} e^{-1}$, so that

$$
\left|S\left(\nu^{n}\right)(\mathbf{x})\right|=\left|\left[\omega_{\mathbf{x}}, \nu^{n}\right]_{\Gamma_{C}}\right| \leq\left\|\omega_{\mathbf{x}}\right\|_{L^{\infty}(\Gamma)}\left|\left[1, \nu^{n}\right]_{\bar{\Gamma}_{C}}\right|
$$

which verifies the uniform boundedness. By Lebesgue's dominated convergence theorem we conclude that $S\left(\nu^{n}\right) \rightarrow S(\tilde{\nu})$ in $L^{2}\left(\Gamma_{S}\right)$.

The following lemma establishes some properties of the solution $\mathbf{u}^{n}$ to the perturbed friction problem (6.2).

6.2. Lemma. I. We have

$$
\operatorname{div}\left(\boldsymbol{\sigma}^{n}\right)=\varepsilon_{n} \varphi \mathbf{u}^{n}-\mathbf{f} \text { in } \Omega
$$

and $S\left(p_{N}^{n}\right)(x)=\left[\omega_{\mathbf{x}}, p_{N}^{n}\right]_{\bar{\Gamma}_{C}}$ is well defined.

II. We have

$$
\left[\mathbf{v}, \mathbf{p}^{n}\right]_{\Gamma_{S}}=a\left(\mathbf{u}^{n}, \mathbf{v}\right)+\varepsilon_{n}\left\langle\varphi \mathbf{u}^{n}, \mathbf{v}\right\rangle_{\Omega}-\langle\mathbf{f}, \mathbf{v}\rangle_{\Omega}-\langle\mathbf{t}, \mathbf{v}\rangle_{\Gamma_{T}}
$$

for all $\mathbf{v} \in H^{1}(\Omega)$ and

$$
\begin{aligned}
{\left[\mathbf{v}-\mathbf{u}^{n}, \mathbf{p}^{n}\right]_{\Gamma_{S}} \geq } & \left\langle\mu S\left(p_{N}^{n}\right),\left|\mathbf{v}_{T}-\mathbf{u}_{T}^{\mathrm{ref}}\right|\right\rangle_{\Gamma_{S}} \\
& -\left\langle\mu S\left(p_{N}^{n}\right),\left|\mathbf{u}_{T}^{n}-\mathbf{u}_{T}^{\mathrm{ref}}\right|\right\rangle_{\Gamma_{S}}
\end{aligned}
$$

for all $\mathbf{v} \in \mathbb{V}=\left\{\mathbf{v} \in H^{1}(\Omega): v_{N} \leq g\right.$ on $\left.\Gamma_{C}\right\}$.

III. Define

$$
\mathbf{w}^{n}=\left\{\begin{array}{cl}
\frac{\mathbf{u}^{n}}{\left\|\mathbf{u}^{n}\right\|_{H^{1}(\Omega)}} & \text { if }\left\|\mathbf{u}^{n}\right\|_{H^{1}(\Omega)} \neq 0 \\
\mathbf{0} & \text { if }\left\|\mathbf{u}^{n}\right\|_{H^{1}(\Omega)}=0
\end{array}\right.
$$

If $\left\|\mathbf{u}^{n}\right\|_{H^{1}(\Omega)} \rightarrow \infty$ when $n \rightarrow \infty$, then there is a convergent subsequence $\mathbf{w}^{n} \rightarrow \mathbf{w}$ in $H^{1}(\Omega)$, where $\mathbf{w} \in \mathcal{N} \cap \mathbb{V}_{0}$ and $\mathbb{V}_{0}=\{\mathbf{v} \in$ $H^{1}(\Omega): v_{N} \leq 0$ on $\left.\Gamma_{C}\right\}$. In fact, we have

$$
a\left(\mathbf{u}^{n}, \mathbf{w}^{n}\right)=\left\|\mathbf{u}^{n}\right\|_{H^{1}(\Omega)} a\left(\mathbf{w}^{n}, \mathbf{w}^{n}\right) \leq C_{17}
$$

where $C_{17}$ is a constant. Moreover, the sequence $\varepsilon_{n}\left\|\mathbf{u}^{n}\right\|_{H^{1}(\Omega)}$ is bounded. 
IV. Let $\Gamma^{*} \subset \Gamma_{C}$. Assume $\left\|\mathbf{u}^{n}\right\|_{H^{1}(\Omega)} \rightarrow \infty$, $\left|\left[1, p_{N}^{n}\right]_{\Gamma^{*}}\right| \rightarrow \infty$ and define $\hat{\mathbf{p}}^{n}=\mathbf{p}^{n} /\left|\left[1, p_{N}^{n}\right]_{\bar{\Gamma}^{*}}\right|$. If $\left[1, \hat{\mathbf{p}}_{N}^{n}\right]_{\Gamma_{C}}$ is a bounded sequence, then

$$
\left\langle S\left(\hat{p}_{N}^{n}\right),\left|\mathbf{w}_{T}^{n}\right|\right\rangle_{\Gamma_{S}} \rightarrow 0
$$

when $n \rightarrow \infty$.

$V$. If $\rho$ is sufficiently small and $\Gamma_{S}$ is continuously differentiable and $\Gamma_{C}$ fulfills the geometrical condition (5.1), then $\left\{\left[1, p_{N}^{n}\right]_{\bar{\Gamma}_{C}}\right\}_{n=1}^{\infty}$ is a bounded sequence. Moreover, if $\left\|\mathbf{u}^{n}\right\|_{H^{1}(\Omega)} \rightarrow \infty$, then

$$
\left\langle S\left(p_{N}^{n}\right),\left|\mathbf{w}_{\text {ref }}^{n}\right|\right\rangle_{\Gamma_{S}} \rightarrow 0
$$

when $n \rightarrow \infty$, where $\mathbf{w}_{\mathrm{ref}}^{n}=\mathbf{u}^{\mathrm{ref}} /\left\|\mathbf{u}^{n}\right\|_{H^{1}(\Omega)}$.

Proof. I. Suppose $\mathbf{z} \in H_{0}^{1}(\Omega)$ and let $\mathbf{v}= \pm \mathbf{z}+\mathbf{u}^{n}$ in the auxiliary problem (6.2). We find

$$
\varepsilon_{n}\left\langle\varphi \mathbf{u}^{n}, \mathbf{z}\right\rangle_{\Omega}+a\left(\mathbf{u}^{n}, \mathbf{z}\right)=\langle\mathbf{f}, \mathbf{z}\rangle_{\Omega} .
$$

Green's formula (5.19) yields $a(\mathbf{u}, \mathbf{z})=-\langle\operatorname{div}(\boldsymbol{\sigma}), \mathbf{z}\rangle_{\Omega}$ so that

$$
\operatorname{div}\left(\boldsymbol{\sigma}^{n}\right)=\varepsilon_{n} \varphi \mathbf{u}^{n}-\mathbf{f} \in L^{2}(\Omega) .
$$

By trace theorems (see [22]), it follows that $\mathbf{p}^{n}=\boldsymbol{\sigma}^{n} \cdot \mathbf{n}-\mathbf{t}$ is well defined in $H^{-1 / 2}(\Gamma)$. Thus $S\left(p_{N}^{n}\right)(x)=\left[\omega_{\mathbf{x}}, p_{N}^{n}\right]_{\bar{\Gamma}_{C}}$ is well defined.

II. From (6.5) follows $\varepsilon_{n} \varphi \mathbf{u}^{n}-\operatorname{div}\left(\boldsymbol{\sigma}^{n}\right)=\mathbf{f}$ in $L^{2}(\Omega)$. Multiply by $\mathbf{v} \in$ $H^{1}(\Omega)$ and use Green's formula (5.19) to obtain

$$
\left[\mathbf{v}, \mathbf{p}^{n}\right]_{\Gamma_{S}}=a\left(\mathbf{u}^{n}, \mathbf{v}\right)+\varepsilon_{n}\left\langle\varphi \mathbf{u}^{n}, \mathbf{v}\right\rangle_{\Omega}-\langle\mathbf{f}, \mathbf{v}\rangle_{\Omega}-\langle\mathbf{t}, \mathbf{v}\rangle_{\Gamma_{T}} .
$$

To prove (6.7), compare (6.2) and (6.6).

III. Since $\left\|\mathbf{w}^{n}\right\|_{H^{1}(\Omega)} \leq 1$, we can use Alaoglu's theorem. Let us assume the sequence $\mathbf{w}^{n}$ converges weakly to $\mathbf{w}$. To prove that $\mathbf{w}$ is a rigid body displacement, let $\mathbf{v}=\mathbf{u}^{\text {ref }}$ in (6.2) to obtain

$$
\begin{aligned}
\left(L, \mathbf{u}^{\text {ref }}-\mathbf{u}^{n}\right) \leq & \varepsilon_{n}\left\langle\varphi \mathbf{u}^{n}, \mathbf{u}^{\text {ref }}-\mathbf{u}^{n}\right\rangle_{\Omega}+a\left(\mathbf{u}^{n}, \mathbf{u}^{\text {ref }}-\mathbf{u}^{n}\right) \\
& +\int_{\Gamma_{S}} \mu S\left(p_{N}^{n}\right)\left|\mathbf{u}_{T}^{n}-\mathbf{u}_{T}^{\mathrm{ref}}\right| \mathrm{d} \Gamma
\end{aligned}
$$

and since $\int_{\Gamma_{S}} \mu S\left(p_{N}^{n}\right)\left|\mathbf{u}_{T}^{n}-\mathbf{u}_{T}^{\mathrm{ref}}\right| \mathrm{d} \Gamma \leq 0$,

$$
\begin{aligned}
\varepsilon_{n}\left\langle\varphi \mathbf{u}^{n}, \mathbf{u}^{n}\right\rangle_{\Omega}+a\left(\mathbf{u}^{n}, \mathbf{u}^{n}\right) \leq & \left(L, \mathbf{u}^{n}\right)-\left(L, \mathbf{u}^{\text {ref }}\right) \\
& +\varepsilon_{n}\left\langle\varphi \mathbf{u}^{n}, \mathbf{u}^{\text {ref }}\right\rangle_{\Omega}+a\left(\mathbf{u}^{n}, \mathbf{u}^{\text {ref }}\right) \\
\leq & C_{17}\left\|\mathbf{u}^{n}\right\|_{H^{1}(\Omega)}
\end{aligned}
$$


for some constant $C_{17}<\infty$. The term $\left(L, \mathbf{u}^{\text {ref }}\right)$ is constant in (6.10), and since $\left\|\mathbf{u}^{n}\right\|_{H^{1}(\Omega)} \rightarrow \infty$ this constant term can be neglected. Let us divide (6.10) by $\left\|\mathbf{u}^{n}\right\|_{H^{1}(\Omega)}$ to conclude

$$
\varepsilon_{n} \varphi_{\min }\left\|\mathbf{u}^{n}\right\|_{H^{1}(\Omega)}\left\langle\mathbf{w}^{n}, \mathbf{w}^{n}\right\rangle_{\Omega}+\left\|\mathbf{u}^{n}\right\|_{H^{1}(\Omega)} a\left(\mathbf{w}^{n}, \mathbf{w}^{n}\right) \leq C_{17} .
$$

This implies $a\left(\mathbf{w}^{n}, \mathbf{w}^{n}\right) \rightarrow 0$ when $\left\|\mathbf{u}^{n}\right\| \rightarrow \infty$, so that

$$
0 \leq a(\mathbf{w}, \mathbf{w}) \leq \lim _{n \rightarrow \infty} a\left(\mathbf{w}^{n}, \mathbf{w}^{n}\right)=0
$$

Thus, from (5.11), $\mathbf{w}=\mathbf{P} \mathbf{w}$ and $\mathbf{w}^{n}-\mathbf{P w}^{n} \rightarrow 0$ in $H^{1}(\Omega)$. Since $\mathcal{N}$ is finite dimensional, we also have $\mathbf{P w}^{n} \rightarrow \mathbf{P w}$ in $H^{1}(\Omega)$, and hence

$$
\mathbf{w}^{n} \rightarrow \mathbf{w} \text { in } H^{1}(\Omega) .
$$

Because of the obstacle, we have $u_{N}^{n} \leq g \leq C_{8}$ on $\Gamma_{C}$ according to (5.4), so that $w_{N}^{n} \leq C_{8} /\left\|\mathbf{u}^{n}\right\|_{H^{1}(\Omega)}$. However $w_{N}^{n} \rightarrow w_{N}$ in $L^{2}\left(\Gamma_{C}\right)$ and $C_{8} /\left\|\mathbf{u}^{n}\right\|_{H^{1}(\Omega)} \rightarrow 0$ uniformly, so that $w_{N} \leq 0$ in the sense of $L^{2}\left(\Gamma_{C}\right)$. But $w_{N}$ is continuous so that we have $w_{N} \leq 0$ everywhere on $\Gamma_{C}$. That is, $\mathbf{w} \in \mathbb{V}_{0}=\left\{\mathbf{v} \in H^{1}(\Omega): v_{N} \leq 0\right.$ on $\left.\Gamma_{C}\right\}$.

From (6.11) follows

$$
\varepsilon_{n} \varphi_{\min }\left\|\mathbf{u}^{n}\right\|_{H^{1}(\Omega)}\left\|\mathbf{w}^{n}\right\|_{L^{2}(\Omega)}^{2} \leq C_{17}
$$

Since $\mathbf{w}^{n} \rightarrow \mathbf{w}$ in $H^{1}(\Omega)$ and norms are equivalent on $\mathcal{N}$, (5.12), we have

$$
\left\|\mathbf{w}^{n}\right\|_{L^{2}(\Omega)}^{2} \geq \frac{1}{2}\|\mathbf{w}\|_{L^{2}(\Omega)}^{2} \geq \frac{C_{13}^{2}}{2}\|\mathbf{w}\|_{H^{1}(\Omega)}^{2}=\frac{C_{13}^{2}}{2}
$$

for large $n$. We find

$$
\varepsilon_{n}\left\|\mathbf{u}^{n}\right\|_{H^{1}(\Omega)} \leq \frac{2 C_{17}}{C_{13}^{2} \varphi_{\min }}
$$

for large $n$, so that $\varepsilon_{n}\left\|\mathbf{u}^{n}\right\|_{H^{1}(\Omega)}$ is bounded.

IV. By Lemma 6.1 we find that $S\left(\hat{p}_{N}^{n}\right) \in L^{2}\left(\Gamma_{S}\right)$, and since $\mathbf{u}^{\text {ref }} \in L^{2}\left(\Gamma_{S}\right)$, we have

$$
\left|\left\langle S\left(\hat{p}_{N}^{n}\right),\left|\mathbf{u}^{\text {ref }}\right|\right\rangle_{\Gamma_{S}}\right| \leq C_{18}
$$

for some constant $C_{18}<\infty$, which yields

$$
\left|\left\langle S\left(\hat{p}_{N}^{n}\right),\left|\mathbf{w}_{\mathrm{ref}}^{n}\right|\right\rangle_{\Gamma_{S}}\right| \leq C_{18} /\left\|\mathbf{u}^{n}\right\|_{H^{1}(\Omega)} \rightarrow 0
$$


when $n \rightarrow \infty$ and where $\mathbf{w}_{\text {ref }}^{n}=\mathbf{u}^{\text {ref }} /\left\|\mathbf{u}^{n}\right\|_{H^{1}(\Omega)}$, so that

$$
\left\langle S\left(\hat{p}_{N}^{n}\right),\left|\mathbf{w}_{\text {ref }}^{n}\right|\right\rangle_{\Gamma_{S}} \rightarrow 0 \text { when } n \rightarrow \infty .
$$

Further,

$$
\left|\left[\mathbf{u}_{T}^{\mathrm{ref}}, \mathbf{p}_{T}^{n}\right]_{\bar{\Gamma}_{S}}\right| \leq\left[\left|\mathbf{u}_{T}^{\mathrm{ref}}\right|,\left|\mathbf{p}_{T}^{n}\right|\right]_{\Gamma_{S}} \leq\left[\left|\mathbf{u}^{\mathrm{ref}}\right|,-\mu S\left(p_{N}^{n}\right)\right]_{\Gamma_{S}}
$$

so that

$$
\left|\left[\mathbf{w}_{\text {ref }, T}^{n}, \hat{\mathbf{p}}_{T}^{n}\right]_{\bar{\Gamma}_{S}}\right| \leq-\mu_{\max }\left\langle\left|\mathbf{w}_{\text {ref }}^{n}\right|, S\left(\hat{p}_{N}^{n}\right)\right\rangle_{\Gamma_{S}}
$$

and thus

$$
\left[\mathbf{w}_{\mathrm{ref}, T}^{n}, \hat{\mathbf{p}}_{T}^{n}\right]_{\bar{\Gamma}_{S}} \rightarrow 0 \text { when } n \rightarrow \infty
$$

according to (6.12).

From III we recall that $a\left(\mathbf{u}^{n}, \mathbf{w}^{n}\right)$ and $\varepsilon_{n}\left\|\mathbf{u}^{n}\right\|_{H^{1}(\Omega)}$ are bounded, so we obtain by $(6.6)$

$$
\begin{aligned}
\left|\left[\mathbf{w}^{n}, \mathbf{p}^{n}\right]_{\Gamma_{S}}\right|= & \mid a\left(\mathbf{u}^{n}, \mathbf{w}^{n}\right)+\varepsilon_{n}\left\langle\varphi \mathbf{u}^{n}, \mathbf{w}^{n}\right\rangle_{\Omega} \\
& -\left\langle\mathbf{f}, \mathbf{w}^{\mathbf{n}}\right\rangle_{\Omega}-\left\langle\mathbf{t}, \mathbf{w}^{\mathbf{n}}\right\rangle_{\Gamma_{T}} \mid \leq C_{20}
\end{aligned}
$$

where $C_{20}$ is constant. Since $\left[u_{N}^{n}, \hat{p}_{N}^{n}\right]_{\bar{\Gamma}_{C}}=\left[g, \hat{p}_{N}^{n}\right]_{\bar{\Gamma}_{C}}$ according to the contact law (5.4), we have

$$
\left|\left[w_{N}^{n}, \hat{p}_{N}^{n}\right]_{\bar{\Gamma}_{C}}\right| \leq \frac{\left|\left[g, \hat{p}_{N}^{n}\right]_{\bar{\Gamma}_{C}}\right|}{\left\|\mathbf{u}^{n}\right\|_{H^{1}(\Omega)}} \leq \frac{C_{8}\left|\left[1, \hat{p}_{N}^{n}\right]_{\bar{\Gamma}_{C}}\right|}{\left\|\mathbf{u}^{n}\right\|_{H^{1}(\Omega)}} \rightarrow 0
$$

when $n \rightarrow \infty$, as the denominator tends to infinity. Thus

$$
\begin{aligned}
\left|\left[\mathbf{w}_{T}^{n}, \hat{\mathbf{p}}_{T}^{n}\right]_{\Gamma_{S}}\right| & \leq\left|\left[\mathbf{w}^{n}, \hat{\mathbf{p}}^{n}\right]_{\Gamma_{S}}\right|+\left|\left[w_{N}^{n}, \hat{p}_{N}^{n}\right]_{\bar{\Gamma}_{C}}\right| \\
& \leq \frac{C_{20}}{\left[1,\left|p_{N}^{n}\right|\right]_{\Gamma^{*}}}+\frac{C_{8}}{\left\|\mathbf{u}^{n}\right\|_{H^{1}(\Omega)}} \rightarrow 0
\end{aligned}
$$

so that

$$
\left[\mathbf{w}_{T}^{n}, \hat{\mathbf{p}}_{T}^{n}\right]_{\Gamma_{S}} \rightarrow 0, \text { when } n \rightarrow \infty
$$

We have

$$
\begin{aligned}
\left|\left\langle\mu S\left(\hat{p}_{N}^{n}\right),\left|\mathbf{w}_{T}^{n}-\mathbf{w}_{\text {ref,T }}^{n}\right|\right\rangle_{\Gamma_{S}}\right|= & \left|\left[\mathbf{w}_{T}^{n}-\mathbf{w}_{\text {ref,T },}^{n}, \hat{\mathbf{p}}_{T}^{n}\right]_{\Gamma_{S}}\right| \\
= & \left|\left[\mathbf{w}_{T}^{n}, \hat{\mathbf{p}}_{T}^{n}\right]_{\Gamma_{S}}-\left[\mathbf{w}_{\mathrm{ref}, T}^{n}, \hat{\mathbf{p}}_{T}^{n}\right]_{\Gamma_{S}}\right| \\
\leq & \left|\left[\mathbf{w}_{T}^{n}, \hat{\mathbf{p}}_{T}^{n}\right]_{\Gamma_{S}}\right| \\
& -\left\langle\mu S\left(\hat{p}_{N}^{n}\right),\left|\mathbf{w}_{\mathrm{ref}, T}^{n}\right|\right\rangle_{\Gamma_{S}} \rightarrow 0
\end{aligned}
$$


when $n \rightarrow \infty$, where the limit follows from (6.12) and (6.15). The sequence $S\left(\hat{p}_{N}^{n}\right) \rightarrow S\left(p_{N}^{*}\right)$ converges in $L^{2}(\Gamma)$ according to Lemma 6.1 and since $\left[1, \hat{\mathbf{p}}_{N}^{n}\right]_{\Gamma_{C}}$ is a bounded sequence, and $\mathbf{w}_{\text {ref }}^{n}=\frac{\mathbf{u}^{\text {ref }}}{\left\|\mathbf{u}^{n}\right\|_{H^{1}(\Omega)}} \rightarrow \mathbf{0}$ in $H^{1}(\Omega)$, so that

$$
\lim _{n \rightarrow \infty}\left\langle\mu S\left(\hat{p}_{N}^{n}\right),\left|\mathbf{w}_{T}^{n}\right|\right\rangle_{\Gamma_{S}}=\lim _{n \rightarrow \infty}\left\langle\mu S\left(\hat{p}_{N}^{n}\right),\left|\mathbf{w}_{T}^{n}-\mathbf{w}_{\mathrm{ref}, T}^{n}\right|\right\rangle_{\Gamma_{S}}=0
$$

V. For the case when $\left\|\mathbf{u}^{n}\right\|_{H^{1}(\Omega)}$ is bounded it follows that $\left[1, p_{N}^{n}\right]_{\bar{\Gamma}_{C}}$ is a bounded sequence by Green's formula (6.6) with an appropriate choice of $\mathbf{v}$. To study the case when $\left\|\mathbf{u}^{n}\right\|_{H^{1}(\Omega)} \rightarrow \infty$ we use proof by contradiction, so let us assume $\left|\left[1, p_{N}^{n}\right]_{\bar{\Gamma}_{C}}\right| \rightarrow \infty$. It follows from Green's formula (6.6) and (5.10) that we can assume

$$
\left|\left[1, p_{N}^{n}\right]_{\bar{\Gamma}_{C}}\right| \leq C_{21}\left\|\mathbf{u}^{n}\right\|_{H^{1}(\Omega)}+C_{22}
$$

for some constants $C_{21} \geq 0$ and $C_{22} \geq 0$.

There is a constant $C_{23}$, which does not depend on $\rho$ so that

$$
\left[1, \omega_{\mathbf{x}}\right]_{\Gamma_{C}} \leq C_{23}
$$

This follows from Lemma 5.2.

Let us now consider $\mathbf{w}=\lim _{n \rightarrow \infty} \mathbf{w}^{n}$ from Lemma 6.2 III. In the following we find a method to prove that the contact force is a a bounded measure on a part of $\Gamma_{C}$ where $|\mathbf{w}|$ has a lower bound. Because of the requirement (5.1), we find that $\mathbf{w}=\mathbf{0}$ only once on the boundary, and Green's formula (9.3) make it possible to limit the measure of the contact force also in a neighbourhood of this point.

Hence, $\mathbf{w} \in \mathcal{N}$, so we find from the requirement (5.1) on $\Gamma_{C}$ that $\mathbf{w}(\hat{\mathbf{x}})=\mathbf{0}$ at most at one point $\hat{\mathbf{x}} \in \Gamma_{C}$. If $\hat{\mathbf{x}}$ exists, let $\mathbf{n}_{\hat{\mathbf{x}}}$ be the outward directed unit normal at this point. Define

$$
\Gamma^{\prime}=\left\{\mathbf{x} \in \bar{\Gamma}_{C}:\left|\mathbf{n}_{\hat{\mathbf{x}}}-\mathbf{n}_{\mathbf{x}}\right| \leq \frac{1}{2\left(1+\mu_{\max } C_{23}\right)}\right\}
$$

where $\mathbf{n}_{\mathbf{x}}$ is the normal vector at $\mathbf{x}$. Moreover, let us denote a neighbourhood of $\hat{\mathbf{x}}$ as

$$
\Lambda_{R}=\left\{\mathbf{x} \in \Gamma_{C}:|\mathbf{x}-\hat{\mathbf{x}}| \leq R\right\}
$$

We assume $\bar{\rho}>0$ is a small constant, so that $A=\Lambda_{2 \bar{\rho}} \subset \Gamma^{\prime}$. Let $B=\Gamma_{C} \backslash \Lambda_{\bar{\rho}}$. Then $A$ and $B$ overlap by a domain of width $\bar{\rho}$, and $A \cup B=\Gamma_{C}$. We assume $\rho<\bar{\rho}$ from now on. This implies

$$
\left|\mathbf{p}_{T}^{n}\right| \leq-\mu_{\max } S\left(p_{N}^{n}\right) \leq-\mu_{\max }\left[\omega_{\mathbf{x}},-p_{N}^{n}\right]_{B}
$$

on $\Gamma_{C} \backslash A$, since $\omega_{\mathbf{x}}=0$ on $\Gamma_{C} \backslash B$ when $\mathbf{x} \in \Gamma_{C} \backslash A$. 
We will first prove that $\left|\left[1, p_{N}^{n}\right]_{\bar{A}}\right| \leq C_{24}\left|\left[1, p_{N}^{n}\right]_{\bar{B}}\right|+C_{25}$ for some constants $C_{24}$ and $C_{25}$, and then we prove that $\left|\left[1, p_{N}^{n}\right]_{\bar{B}}\right|$ is bounded. If $\hat{\mathbf{x}}$ exists, this proves that $\left|\left[1, p_{N}^{n}\right]_{\Gamma_{C}}\right|$ is bounded. If $\hat{\mathbf{x}}$ does not exist, then the second half of this proof with $B=\Gamma_{C}$ proves the boundedness of $\left|\left[1, p_{N}^{n}\right]_{\Gamma_{C}}\right|$.

Let $\overline{\mathbf{n}}$ denote the displacement field with $\overline{\mathbf{n}}=\mathbf{n}_{\hat{\mathbf{x}}}$ at each point. Then $\overline{\mathbf{n}} \in \mathcal{N}$, so that Green's formula (9.3) yields

$$
\begin{aligned}
{\left[\overline{\mathbf{n}}, \mathbf{p}^{n}\right]_{\bar{A}}=} & -\left[\overline{\mathbf{n}}, \mathbf{p}^{n}\right]_{\bar{\Gamma}_{C} \backslash \bar{A}}+\varepsilon_{n}\left\langle\varphi \mathbf{u}^{n}, \overline{\mathbf{n}}\right\rangle_{\Omega} \\
& -\langle\mathbf{f}, \overline{\mathbf{n}}\rangle_{\Omega}-\langle\mathbf{t}, \overline{\mathbf{n}}\rangle_{\Gamma}
\end{aligned}
$$

Since $\varepsilon_{n}\left\langle\varphi \mathbf{u}^{n}, \overline{\mathbf{n}}\right\rangle_{\Omega}$ is bounded according to Lemma 6.2 III, we conclude that

$$
\begin{aligned}
\left|\left[\overline{\mathbf{n}}, \mathbf{p}^{n}\right]_{\bar{A}}\right| & \leq C_{26}\left|\left[\overline{\mathbf{n}}, \mathbf{p}^{n}\right]_{\bar{\Gamma}_{C} \backslash A}\right|+C_{27} \\
& \leq C_{28}\left|\left[1, p_{N}^{n}\right]_{\bar{\Gamma}_{C} \backslash A}\right|+C_{29}\left|\left[1,\left|\mathbf{p}_{T}^{n}\right|\right]_{\bar{\Gamma}_{C} \backslash A}\right|+C_{27} \\
& \leq C_{28}\left|\left[1, p_{N}^{n}\right]_{B}\right|+C_{29} \mu_{\max }\left|\left[1,\left|\left[\omega_{\mathbf{x}}, p_{N}^{n}\right]_{B}\right|\right]_{\bar{\Gamma}_{C} \backslash A}\right|+C_{27} \\
& \leq C_{30}\left|\left[1, p_{N}^{n}\right]_{B}\right|+C_{27}
\end{aligned}
$$

for some constants $C_{26}, C_{27}, C_{28}, C_{29}$ and $C_{30}$. We have

$$
\begin{aligned}
{\left[1,\left|\mathbf{p}^{n}\right|\right]_{A} \leq } & {\left[1,-p_{N}^{n}-\mu_{\max } S\left(p_{N}^{n}\right)\right]_{A} } \\
\leq & {\left[1,-p_{N}^{n}\right]_{A}+\mu_{\max }\left[1,\left[\omega_{\mathbf{x}},-p_{N}^{n}\right]_{\Gamma_{C}}\right]_{A} } \\
\leq & {\left[1,-p_{N}^{n}\right]_{A}+\mu_{\max }\left[\omega_{\mathbf{x}}, 1\right]_{A}\left[1,-p_{N}^{n}\right]_{\bar{\Gamma}_{C}} } \\
\leq & {\left[1,-p_{N}^{n}\right]_{A}+\mu_{\max } C_{23}\left[1,-p_{N}^{n}\right]_{\bar{\Gamma}_{C}} } \\
\leq & \left(1+\mu_{\max } C_{23}\right)\left[1,-p_{N}^{n}\right]_{A} \\
& +\mu_{\max } C_{23}\left[1,-p_{N}^{n}\right]_{B}
\end{aligned}
$$

and $|\mathbf{n}-\overline{\mathbf{n}}| \leq \frac{1}{2\left(1+\mu_{\max } C_{23}\right)}$ on $A$, so that

$$
\begin{aligned}
\left|\left[1, p_{N}^{n}\right]_{A}\right| & =\left|\left[\mathbf{n}, \mathbf{p}^{n}\right]_{A}\right| \leq\left[\overline{\mathbf{n}}, \mathbf{p}^{n}\right]_{A}+\left[|\mathbf{n}-\overline{\mathbf{n}}|,\left|\mathbf{p}^{n}\right|\right]_{A} \\
& \leq\left[\overline{\mathbf{n}}, \mathbf{p}^{n}\right]_{A}+\left[\frac{1}{2\left(1+\mu_{\max } C_{23}\right.},\left|\mathbf{p}^{n}\right|\right]_{A} \\
& \leq C_{30}\left|\left[1, p_{N}^{n}\right]_{\bar{B}}\right|+C_{27}+\left[\frac{1}{2\left(1+\mu_{\max } C_{23}\right.},\left|\mathbf{p}^{n}\right|\right]_{A} \\
& \leq C_{30}\left|\left[1, p_{N}^{n}\right]_{\bar{B}}\right|+C_{27}+\frac{1}{2\left(1+\mu_{\max } C_{23}\right.}\left[1,\left|\mathbf{p}^{n}\right|\right]_{A} \\
& \leq 1 / 2\left|\left[1, p_{N}^{n}\right]_{A}\right|+C_{31}\left|\left[1, p_{N}^{n}\right]_{B}\right|+C_{27}
\end{aligned}
$$

and we find

$$
\left|\left[1, p_{N}^{n}\right]_{\bar{A}}\right| \leq C_{24}\left|\left[1, p_{N}^{n}\right]_{\bar{B}}\right|+C_{25}
$$

where $C_{24}$ and $C_{25}$ are constants. 
We assumed $\mid\left[1, p_{N}^{n}\right]_{\bar{\Gamma}_{C}} \rightarrow \infty$, so we conclude that $\mid\left[1, p_{N}^{n}\right]_{B} \rightarrow \infty$ when $n \rightarrow \infty$. Since $\mathbf{w} \neq \mathbf{0}$ on $\bar{B}$, we have $|\mathbf{w}| \geq C_{32}>0$ for some constant $C_{32}$.

Cover the surface $\bar{B}$ by disjoint patches, where each patch is contained within a circle of radius $\rho / 4$ in the Euclidean metric (so that the distance $|\mathbf{x}-\mathbf{y}|$ between two points $\mathbf{x}$ and $\mathbf{y}$ in the patch is at most $\rho / 2)$. Since the relative boundary of $B$ is Lipschitz we can assume that the partition is done so that each patch has an surface area greater than $\eta \rho^{2}$, where $\eta>0$ is a constant. That is, $\eta$ does not depend on $\rho$.

Note also that the every point of $\bar{B}$ is covered only once, since the patches were assumed to be disjoint. Now, let $\Pi$ be one of these patches.

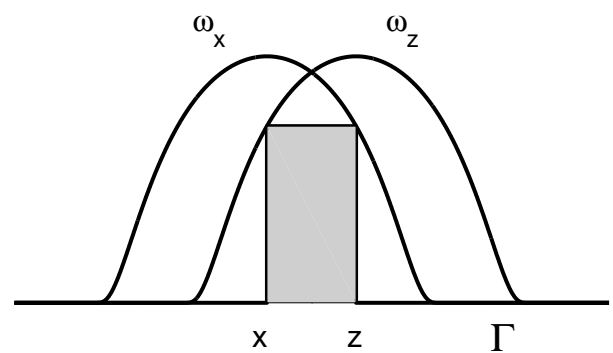

Figure 6.1: We have $\omega_{\mathbf{z}}(\mathbf{x}) \geq \frac{C^{*}}{\rho^{2}} e^{-4 / 3}$ if $\mathbf{x} \in \Pi$ and $\mathbf{z} \in \Pi$.

We have, see Figure 6.1, $\omega_{\mathbf{z}}(\mathbf{x}) \geq C_{33} / \rho^{2}$ if $\mathbf{x} \in \Pi$ and $\mathbf{z} \in \Pi$, for some constant $C_{33}=C^{*} e^{-4 / 3}>0$. Hence

$$
\begin{aligned}
-\left\langle S\left(p_{N}^{n}\right),|\mathbf{w}|\right\rangle_{\Pi} & =\left\langle\left[\omega_{\mathbf{x}},-p_{N}^{n}\right]_{\bar{\Gamma}_{C}},|\mathbf{w}|\right\rangle_{\Pi} \\
& \geq\left\langle\left[\omega_{\mathbf{x}},-p_{N}^{n}\right]_{\Pi},|\mathbf{w}|\right\rangle_{\Pi} \\
& \geq\left\langle\left[\frac{C_{33}}{\rho^{2}},-p_{N}^{n}\right]_{\Pi},|\mathbf{w}|\right\rangle_{\Pi} \\
& =\frac{C_{33}}{\rho^{2}}\left[1,-p_{N}^{n}\right]_{\Pi}\langle 1,|\mathbf{w}|\rangle_{\Pi} \\
& \geq \frac{C_{33} C_{32}}{\rho^{2}}\left[1,-p_{N}^{n}\right]_{\Pi}\langle 1,1\rangle_{\Pi} \\
& \geq \frac{C_{33} C_{32}}{\rho^{2}}\left[1,-p_{N}^{n}\right]_{\Pi} \eta \rho^{2} \\
& =\eta C_{33} C_{32}\left[1,-p_{N}^{n}\right]_{\Pi} .
\end{aligned}
$$


We obtain by summing over all patches

$$
-\left\langle S\left(p_{N}^{n}\right),|\mathbf{w}|\right\rangle_{\bar{B}} \geq \eta C_{33} C_{32}\left[1,-p_{N}^{n}\right]_{\bar{B}} .
$$

We have

$$
\begin{aligned}
\left|\left[1, p_{N}^{n}\right]_{\bar{\Gamma}_{C}}\right| & \leq\left|\left[1, p_{N}^{n}\right]_{\bar{A}}\right|+\left|\left[1, p_{N}^{n}\right]_{\bar{B}}\right| \\
& \leq\left(1+C_{24}\right)\left|\left[1, p_{N}^{n}\right]_{\bar{B}}\right|+C_{25}
\end{aligned}
$$

so that $\hat{\mathbf{p}}^{n}=\mathbf{p}^{n} /\left|\left[1, p_{N}^{n}\right]\right|_{\bar{B}}$ is a measure on $\bar{\Gamma}_{C}$ with uniformly bounded total variation. Now, let $\mathbf{p}^{*}$ be the weak limit of $\hat{\mathbf{p}}^{n}=$ $\mathbf{p}^{n} /\left|\left[1, p_{N}^{n}\right]\right|_{\bar{B}}$ in the sense of measures according to (6.3). Expressed in $\hat{\mathbf{p}}^{n}$, we have from $(6.17)$

$$
-\left\langle S\left(\hat{p}_{N}^{n}\right),|\mathbf{w}|\right\rangle_{\bar{B}} \geq \eta C_{33} C_{32}
$$

and by taking limits $n \rightarrow \infty$ we obtain

$$
-\left\langle S\left(\hat{p}_{N}^{*}\right),|\mathbf{w}|\right\rangle_{\bar{B}} \geq \eta C_{33} C_{32}
$$

From (6.8) we have

$$
\left\langle S\left(\hat{p}_{N}^{*}\right),\left|\mathbf{w}_{T}\right|\right\rangle_{\Gamma_{S}}=\lim _{n \rightarrow \infty}\left\langle S\left(\hat{p}_{N}^{n}\right),\left|\mathbf{w}_{T}^{n}\right|\right\rangle_{\Gamma_{S}}=0
$$

so that $\left\langle S\left(\hat{p}_{N}^{*}\right),\left|\mathbf{w}_{T}\right|\right\rangle_{\bar{B}}=0$ and hence

$$
\left\langle S\left(\hat{p}_{N}^{*}\right), w_{N}\right\rangle_{\bar{B}} \geq-\left\langle S\left(\hat{p}_{N}^{*}\right),|\mathbf{w}|\right\rangle_{\bar{B}} \geq \eta C_{33} C_{32} .
$$

We now use

$$
\begin{aligned}
\left\langle S\left(p_{N}^{*}\right), w_{N}\right\rangle_{\bar{B}} & =\left\langle\left[\omega_{\mathbf{x}}, p_{N}^{*}\right]_{\bar{\Gamma}_{C}}, w_{N}\right\rangle_{\bar{B}} \\
& =\left[\left\langle\omega_{\mathbf{x}}, w_{N}\right\rangle_{\bar{B}}, p_{N}^{*}\right]_{\bar{\Gamma}_{C}}
\end{aligned}
$$

and apply Lemma 5.2,

$$
\left|\left[\left\langle\omega_{\mathbf{x}}, w_{N}\right\rangle_{\bar{B}}, p_{N}^{*}\right]_{\bar{\Gamma}_{C}}-\left[\Phi w_{N}, p_{N}^{*}\right]_{\bar{\Gamma}_{C}}\right| \leq C_{34} \rho
$$

where $\Phi<\Phi_{\max }<\infty$ and $C_{34}$ is a constant. Here we use that the set $\left\{\mathbf{w} \in \mathcal{N}:\|\mathbf{w}\|_{H^{1}(\Omega)}=1\right\}$ is uniformly Lipschitz.

Thus, we find from (6.18)

$$
\left[w_{N}, \hat{p}_{N}^{*}\right]_{\bar{\Gamma}_{C}} \geq\left(\eta C_{33} C_{32}-C_{34} \rho\right) / \Phi_{\max }
$$

so that

$$
\left[w_{N}, \hat{p}_{N}^{*}\right]_{\Gamma_{C}}>0
$$


when $\rho$ is sufficiently small.

To find a contradiction, let us also prove

$$
\left[w_{N}, \hat{p}_{N}^{*}\right]_{\Gamma_{C}}=0
$$

Since $a\left(\mathbf{u}^{n}, \mathbf{w}\right)=0$, we obtain from equation (6.6),

$$
\left|\left[\mathbf{w}, \mathbf{p}^{n}\right]_{\Gamma_{S}}\right|=\left|\varepsilon_{n}\left\langle\varphi \mathbf{u}^{n}, \mathbf{w}\right\rangle_{\Omega}-\langle\mathbf{f}, \mathbf{w}\rangle_{\Omega}-\langle\mathbf{t}, \mathbf{w}\rangle_{\Gamma}\right| \leq C_{35}
$$

where $C_{35}$ is a constant, so that

$$
\left|\left[\mathbf{w}, \mathbf{p}^{*}\right]_{\Gamma_{S}}\right| \leq \lim _{n \rightarrow \infty}\left(C_{35} /\left|\left[1, p_{N}^{n}\right]_{\bar{B}}\right|\right)=0
$$

that is

$$
\left[w_{N}, p_{N}^{*}\right]_{\bar{\Gamma}_{C}}+\left[\mathbf{w}_{T}, \mathbf{p}_{T}^{*}\right]_{\bar{\Gamma}_{S}}=\left[\mathbf{w}, \mathbf{p}^{*}\right]_{\Gamma_{S}}=0
$$

Since $S\left(\hat{p}_{N}^{n}\right) \rightarrow S\left(p_{N}^{*}\right)$ in $L^{2}(\Gamma)$, we obtain by Cauchy-Schwarz inequality and (6.8)

$$
\begin{aligned}
\left|\left[\mathbf{w}_{T}, \hat{\mathbf{p}}_{T}^{n}\right]_{\Gamma_{S}}\right| \leq & \left|\left\langle\mu S\left(\hat{p}_{N}^{n}\right),\left|\mathbf{w}_{T}\right|\right\rangle_{\Gamma_{S}}\right| \\
& \rightarrow\left|\left\langle\mu S\left(p_{N}^{*}\right),\left|\mathbf{w}_{T}\right|\right\rangle_{\Gamma_{S}}\right|=0
\end{aligned}
$$

when $n \rightarrow \infty$. As $\hat{\mathbf{p}}^{n} \rightarrow \mathbf{p}^{*}$ weakly, in the sense of measures, and $w_{T}$ is continuous as $\mathbf{w} \in \mathcal{N}$ we obtain $\left[\mathbf{w}_{T}, \mathbf{p}_{T}^{*}\right]=0$ from $(6.21)$, and thus (6.20) yields

$$
\left[w_{N}, p_{N}^{*}\right]_{\bar{\Gamma}_{C}}=0
$$

Now (6.22) contradicts (6.19), which proves that $\left\{\left[1, p_{N}^{n}\right]_{\bar{B}}\right\}_{n=1}^{\infty}$ is bounded, and also the boundedness of $\left[1, p_{N}^{n}\right]_{\bar{\Gamma}_{C}}$.

To prove $(6.9)$, note that $S\left(p_{N}^{n}\right) \in L^{2}\left(\Gamma_{S}\right)$ and $\left\|S\left(p_{N}^{n}\right)\right\|_{L^{2}\left(\Gamma_{S}\right)}$ is bounded if $\left\{\left[1, p_{N}^{n}\right]_{\bar{\Gamma}_{C}}\right\}_{n=1}^{\infty}$ is bounded according to Lemma 6.1. Since also $\mathbf{u}^{\text {ref }} \in L^{2}\left(\Gamma_{S}\right)$ we find

$$
\left|\left\langle S\left(p_{N}^{n}\right),\left|\mathbf{u}^{\mathrm{ref}}\right|\right\rangle_{\Gamma_{S}}\right| \leq C_{36}
$$

for some constant $C_{36}<\infty$. We find

$$
\left|\left\langle S\left(p_{N}^{n}\right),\left|\mathbf{w}_{\text {ref }}^{n}\right|\right\rangle_{\Gamma_{S}}\right| \leq \frac{C_{36}}{\left\|\mathbf{u}^{n}\right\|_{H^{1}(\Omega)}} \rightarrow 0
$$

when $n \rightarrow \infty$. 
We now state the main theorem.

6.3. Theorem. Lack of solutions to the friction problem (5.13) implies that for every function $\varphi \in L^{\infty}(\Omega)$ such that $\varphi \geq \varphi_{\min }>0$ there exists a rigid body displacement $\mathbf{w} \in \mathbb{V}_{0}=\left\{\mathbf{v} \in H^{1}(\Omega): v_{N} \leq 0\right.$ on $\left.\Gamma_{C}\right\}$, a contact force field $\mathbf{p}$ of finite total variation on $\Gamma_{S}$ such that $-p_{N}$ is a positive measure with support in $\bar{\Gamma}_{C}$ and a constant $\alpha \geq 0$ such that

$I$.

$$
[\mathbf{v}, \mathbf{p}]_{\Gamma_{S}}=\alpha\langle\varphi \mathbf{w}, \mathbf{v}\rangle_{\Omega}-\langle\mathbf{f}, \mathbf{v}\rangle_{\Omega}-\langle\mathbf{t}, \mathbf{v}\rangle_{\Gamma_{T}}
$$

for all $\mathbf{v} \in \mathcal{N}$.

II.

$$
[\mathbf{v}-\mathbf{w}, \mathbf{p}]_{\Gamma_{S}}-\left\langle\mu S\left(p_{N}\right),\left|\mathbf{v}_{T}\right|-\left|\mathbf{w}_{T}\right|\right\rangle_{\Gamma_{S}} \geq 0
$$

for all $\mathbf{v} \in\left\{\mathbf{v} \in \mathbb{V}_{0}: \mathbf{v}\right.$ continuous on $\left.\Gamma_{S}\right\}$.

provided $\rho$ is small, $\Gamma_{S}$ is $C^{2}$ and $\Gamma_{C}$ satisfies the geometrical condition (5.1).

To prove this theorem we shall consider the sequence of solutions $\left\{\mathbf{u}^{n}\right\}_{n=1}^{\infty}$ to (6.2) obtained when $\varepsilon_{n} \rightarrow 0$. We shall construct a solution to the friction problem (5.13) if $\left\|\mathbf{u}^{n}\right\|_{H^{1}(\Omega)}$ is a bounded sequence. This is a main point of the theorem, from which we conclude that lack of solutions to the original problem (5.13) implies that a subsequence of $\left\|\mathbf{u}^{n}\right\|_{H^{1}(\Omega)}$ tends to infinity. This enable us to prove that (6.23) and (6.24) are fulfilled for some rigid body motion $\mathbf{w}$ and some contact force field $\mathbf{p}$ if the friction problem (5.13) lacks solutions.

Theorem (6.3) concerns lack of solutions, and find conditions that are fulfilled when there is no solution to the problem (5.13). If these conditions are impossible to satisfy because of the choice of the applied force, then we conclude that there exists a solution. Thus, we have a sufficient condition for the existence of a solution to the friction problem (5.13).

Proof. Consider the sequence of solutions $\left\{\mathbf{u}^{n}\right\}_{n=1}^{\infty}$ of (6.2).

If $\left\|\mathbf{u}^{n}\right\|_{H^{1}(\Omega)}$ is bounded for some choice of $\varphi$, then we shall construct a solution of (5.13). Otherwise we shall prove that, for every admissible choice of $\varphi,(6.23)$ and (6.24) are fulfilled for some rigid body motion $\mathbf{w}$ and some contact force field $\mathbf{p}$. By definition of $\mathbf{u}^{n}$ we have

$$
\begin{aligned}
\left(L, \mathbf{v}-\mathbf{u}^{n}\right) \leq & \varepsilon_{n}\left\langle\varphi \mathbf{u}^{n}, \mathbf{v}-\mathbf{u}^{n}\right\rangle_{\Omega}+a\left(\mathbf{u}^{n}, \mathbf{v}-\mathbf{u}^{n}\right) \\
& +\left\langle\mu S\left(p_{N}^{n}\right),\left|\mathbf{u}_{T}^{n}-\mathbf{u}_{T}^{\mathrm{ref}}\right|-\left|\mathbf{v}_{T}-\mathbf{u}_{T}^{\mathrm{ref}}\right|\right\rangle_{\Gamma_{S}}
\end{aligned}
$$

for all $\mathbf{v} \in \mathbb{V}$. 
Let us first consider the case when $\left\|\mathbf{u}^{n}\right\|_{H^{1}(\Omega)}$ is bounded. Then there is a weakly convergent subsequence of $\left\{\mathbf{u}^{n}\right\}_{n=1}^{\infty}$ and without loss of generality we can assume $\mathbf{u}^{n} \rightarrow \mathbf{u}^{*}$ in $H^{1}(\Omega)$.

Lemma $6.2 \mathrm{~V}$ implies that $\left[1, p_{N}^{n}\right]_{\bar{\Gamma}_{C}}$ is bounded. Thus $p_{N}^{n} \rightarrow p_{N}^{*}$ weakly in the sense of measures (6.3), and $S\left(p_{N}^{n}\right)$ converges strongly in $L^{2}\left(\Gamma_{S}\right)$ according to Lemma 6.1 , and hence

$$
\left\langle S\left(p_{N}^{n}\right),\left|\mathbf{v}_{T}-\mathbf{u}_{T}^{\mathrm{ref}}\right|\right\rangle_{\Gamma_{S}} \rightarrow\left\langle S\left(p_{N}^{*}\right),\left|\mathbf{v}_{T}-\mathbf{u}_{T}^{\mathrm{ref}}\right|\right\rangle_{\Gamma_{S}} .
$$

The trace theorem implies that $\mathbf{u}_{T}^{n} \rightarrow \mathbf{u}_{T}^{*}$ in $L^{2}\left(\Gamma_{S}\right)$, so that we obtain

$$
\liminf _{n \rightarrow \infty}\left\langle S\left(p_{N}^{n}\right),\left|\mathbf{u}_{T}^{n}-\mathbf{u}_{T}^{\mathrm{ref}}\right|\right\rangle_{\Gamma_{S}} \rightarrow\left\langle S\left(p_{N}^{*}\right),\left|\mathbf{u}_{T}^{*}-\mathbf{u}_{T}^{\mathrm{ref}}\right|\right\rangle_{\Gamma_{S}} .
$$

Also $\varepsilon_{n}\left\langle\varphi \mathbf{u}^{n}, \mathbf{v}-\mathbf{u}^{n}\right\rangle_{\Omega} \rightarrow 0$. Thus, let $n \rightarrow \infty$ in (6.25),

$$
\begin{aligned}
\left(L, \mathbf{v}-\mathbf{u}^{*}\right) \leq & a\left(\mathbf{u}^{*}, \mathbf{v}-\mathbf{u}^{*}\right)+\left\langle S\left(p_{N}^{*}\right),\left|\mathbf{u}_{T}^{*}-\mathbf{u}_{T}^{\mathrm{ref}}\right|\right\rangle_{\Gamma_{C}} \\
& -\left\langle S\left(p_{N}^{*}\right),\left|\mathbf{v}_{T}-\mathbf{u}_{T}^{\mathrm{ref}}\right|\right\rangle_{\Gamma_{C}}
\end{aligned}
$$

for all $\mathbf{v} \in \mathbb{V}=\left\{\mathbf{v} \in H^{1}(\Omega): v_{N} \leq g\right.$ on $\left.\Gamma_{C}\right\}$. Thus $\mathbf{u}^{*}$ is a solution to (5.13). And $\mathbf{p}^{*}$ is a corresponding contact force, since Green's formula (5.19) and (5.14) give

$$
\begin{aligned}
{\left[\mathbf{v}, \mathbf{p}^{*}\right]_{\Gamma_{S}}+(L, \mathbf{v}) } & =\lim _{n \rightarrow \infty}\left(\left[\mathbf{v}, \mathbf{p}^{n}\right]_{\Gamma_{S}}+(L, \mathbf{v})\right) \\
& =\lim _{n \rightarrow \infty}\left(\varepsilon_{n}\left\langle\varphi \mathbf{u}^{n}, \mathbf{v}\right\rangle_{\Omega}+a\left(\mathbf{u}^{n}, \mathbf{v}\right)\right) \\
& =a\left(\mathbf{u}^{*}, \mathbf{v}\right) \\
& =\left[\mathbf{v}, \boldsymbol{\sigma}^{*} \cdot \mathbf{n}\right]_{\Gamma}-\left\langle\operatorname{div}\left(\boldsymbol{\sigma}^{*}\right), \mathbf{v}\right\rangle_{\Omega}
\end{aligned}
$$

where $\boldsymbol{\sigma}^{*}$ is the stress tensor corresponding to $\mathbf{u}^{*}$. We find $\mathbf{p}^{*}=\boldsymbol{\sigma}^{*} \cdot \mathbf{n}$ on $\Gamma_{S}$.

Now consider the case when $\left\|\mathbf{u}^{n}\right\|_{H^{1}(\Omega)}$ is not bounded. Without loss of generality we assume $\left\|\mathbf{u}^{n}\right\|_{H^{1}(\Omega)} \rightarrow \infty$ and, by Lemma 6.2 III,

$$
\mathbf{w}^{n}=\frac{\mathbf{u}^{n}}{\left\|\mathbf{u}^{n}\right\|_{H^{1}(\Omega)}} \rightarrow \mathbf{w} \in \mathcal{N} \cap \mathbb{V}_{0}
$$

The sequence $\varepsilon_{n}\left\|\mathbf{u}^{n}\right\|_{H^{1}(\Omega)}$ is bounded, so let us assume $\varepsilon_{n}\left\|\mathbf{u}^{n}\right\|_{H^{1}(\Omega)} \rightarrow \alpha$. Lemma $6.2 \mathrm{~V}$ states that the sequence $\left[1, p_{N}^{n}\right]_{\bar{\Gamma}_{C}}$ is bounded and thus let us assume $p_{N}^{n} \rightarrow p_{N}$ weakly in the sense of measures (6.3). The mollified contact force $S\left(p_{N}^{n}\right)$ converges strongly in $L^{2}\left(\Gamma_{S}\right)$ according to Lemma 6.1 .

To prove $(6.23)$, let $\mathbf{v} \in \mathcal{N}$ in $(6.6)$

$$
[\mathbf{v}, \mathbf{p}]_{\Gamma_{S}}=\varepsilon_{n}\left\langle\varphi \mathbf{u}^{n}, \mathbf{v}\right\rangle_{\Omega}-\langle\mathbf{f}, \mathbf{v}\rangle_{\Omega}-\langle\mathbf{t}, \mathbf{v}\rangle_{\Gamma_{T}}
$$

and then (6.23) follows as $n \rightarrow \infty$. To prove (6.24) we use (6.7) which implies

$$
\begin{aligned}
{\left[\left\|\mathbf{u}^{n}\right\|_{H^{1}(\Omega)} \mathbf{v}-\mathbf{u}^{n}, \mathbf{p}^{n}\right]_{\Gamma_{S}} \geq } & \left\langle\mu S\left(p_{N}^{n}\right),\left|\left\|\mathbf{u}^{n}\right\|_{H^{1}(\Omega)} \mathbf{v}_{T}-\mathbf{u}_{T}^{\mathrm{ref}}\right|\right\rangle_{\Gamma_{S}} \\
& -\left\langle\mu S\left(p_{N}^{n}\right),\left|\mathbf{u}_{T}^{n}-\mathbf{u}_{T}^{\mathrm{ref}}\right|\right\rangle_{\Gamma_{S}}
\end{aligned}
$$


for all $\mathbf{v} \in H^{1}(\Omega)$ such that $v_{N} \leq 0$. This $\mathbf{v}$ also satisfies $\left\|\mathbf{u}^{n}\right\|_{H^{1}(\Omega)} v_{N} \leq g$ since $g \geq 0$. The triangle inequality and division by $\left\|\mathbf{u}^{n}\right\|_{H^{1}(\Omega)}$ give

$$
\begin{aligned}
{\left[\mathbf{v}-\mathbf{w}^{n}, \mathbf{p}^{n}\right]_{\Gamma_{S}} \geq } & \left\langle\mu S\left(p_{N}^{n}\right),\left|\mathbf{v}_{T}\right|\right\rangle_{\Gamma_{S}}-\left\langle\mu S\left(p_{N}^{n}\right),\left|\mathbf{w}_{T}^{n}\right|\right\rangle_{\Gamma_{S}} \\
& -2\left|\left\langle\mu S\left(p_{N}^{n}\right),\left|\mathbf{w}_{T}^{\mathrm{ref}}\right|\right\rangle_{\Gamma_{S}}\right| .
\end{aligned}
$$

Now we let $n \rightarrow \infty$. From the condition that $\mathbf{v}$ is continuous on $\Gamma_{S}$ we find that $\left[\mathbf{v}, \mathbf{p}^{n}\right]_{\Gamma_{S}} \rightarrow[\mathbf{v}, \mathbf{p}]_{\Gamma_{S}}$ and we have also

$$
\left\langle\mu S\left(p_{N}^{n}\right),\left|\mathbf{w}_{T}^{\mathrm{ref}}\right|\right\rangle_{\Gamma_{S}} \rightarrow 0
$$

according to $(6.9)$ and

$$
\left\langle\mu S\left(p_{N}^{n}\right),\left|\mathbf{w}_{T}^{n}\right|\right\rangle_{\Gamma_{S}} \rightarrow\left\langle\mu S\left(p_{N}\right),\left|\mathbf{w}_{T}\right|\right\rangle_{\Gamma_{S}}
$$

and

$$
\left\langle\mu S\left(p_{N}^{n}\right),\left|\mathbf{v}_{T}\right|\right\rangle_{\Gamma_{S}} \rightarrow\left\langle\mu S\left(p_{N}\right),\left|\mathbf{v}_{T}\right|\right\rangle_{\Gamma_{S}}
$$

according to Lemma 6.1. To prove (6.24) it remains to show that

$$
\liminf _{n \rightarrow \infty}\left[\mathbf{w}^{n}, \mathbf{p}^{n}\right]_{\Gamma_{S}} \geq[\mathbf{w}, \mathbf{p}]_{\Gamma_{S}}
$$

We use (6.6), which yields

$$
\begin{aligned}
{\left[\mathbf{w}^{n}, \mathbf{p}^{n}\right]_{\Gamma_{S}}=} & \left\|\mathbf{u}^{n}\right\|_{H^{1}(\Omega)} a\left(\mathbf{w}^{n}, \mathbf{w}^{n}\right)+\varepsilon_{n}\left\langle\varphi \mathbf{u}^{n}, \mathbf{w}^{n}\right\rangle_{\Omega} \\
& -\left\langle\mathbf{f}, \mathbf{w}^{n}\right\rangle_{\Omega}-\left\langle\mathbf{t}, \mathbf{w}^{n}\right\rangle_{\Gamma_{T}} \\
\geq & \varepsilon_{n}\left\langle\varphi \mathbf{u}^{n}, \mathbf{w}^{n}\right\rangle_{\Omega}-\left\langle\mathbf{f}, \mathbf{w}^{n}\right\rangle_{\Omega}-\left\langle\mathbf{t}, \mathbf{w}^{n}\right\rangle_{\Gamma_{T}} \\
\geq & \varepsilon_{n}\left\|\mathbf{u}^{n}\right\|_{H^{1}(\Omega)}\left\langle\varphi \mathbf{w}^{n}, \mathbf{w}^{n}\right\rangle_{\Omega}-\left\langle\mathbf{f}, \mathbf{w}^{n}\right\rangle_{\Omega}-\left\langle\mathbf{t}, \mathbf{w}^{n}\right\rangle_{\Gamma_{T}}
\end{aligned}
$$

and if we let $n \rightarrow \infty$

$$
\liminf _{n \rightarrow \infty}\left[\mathbf{w}^{n}, \mathbf{p}^{n}\right]_{\Gamma_{S}} \geq \alpha\langle\varphi \mathbf{w}, \mathbf{w}\rangle_{\Omega}-\langle\mathbf{f}, \mathbf{w}\rangle_{\Omega}-\langle\mathbf{t}, \mathbf{w}\rangle_{\Gamma_{T}}
$$

and since $\alpha\langle\varphi \mathbf{w}, \mathbf{w}\rangle_{\Omega}-\langle\mathbf{f}, \mathbf{w}\rangle_{\Omega}-\langle\mathbf{t}, \mathbf{w}\rangle_{\Gamma_{T}}=[\mathbf{w}, \mathbf{p}]_{\bar{\Gamma}_{S}}$ according to (6.23) we find

$$
\liminf _{n \rightarrow \infty}\left[\mathbf{w}^{n}, \mathbf{p}^{n}\right]_{\Gamma_{S}} \geq \alpha\langle\varphi \mathbf{w}, \mathbf{w}\rangle_{\Omega}-\langle\mathbf{f}, \mathbf{w}\rangle_{\Omega}-\langle\mathbf{t}, \mathbf{w}\rangle_{\Gamma_{T}}=[\mathbf{w}, \mathbf{p}]_{\Gamma_{S}}
$$

as required, that is $(6.26)$.

remark 1: Two dimensional friction problems with nonlocal friction. For computer efficiency, it is often useful to consider symmetry of the geometry while doing numerical modelling. This results in lower dimensional problems. The report [31] considers two dimensional noncoercive incremental friction problems with nonlocal friction. The Theorem 6.3 is the 
same, and the proof is similar. Also, the requirement (5.1) is always satisfied in two dimensions, because a nonzero rigid body displacement can only vanish at one point.

remark 2: Interpretation of Theorem 6.3. The conditions in Theorem 6.3 can be interpreted similar to the conditions in the discrete case. See Chapter 2 section 3 .

remark 3: The condition on $\mathbf{v}$ to be continuous on $\Gamma_{S}$ in $(6.24)$ can be relaxed. We only need to require that $v_{N}$ is continuous on $\bar{\Gamma}_{C}$. The reason is that $\mathbf{p}_{T}^{n} \rightarrow \mathbf{p}_{T}$ weakly in $L^{2}\left(\Gamma_{S}\right)$ because $\left|\mathbf{p}_{T}^{n}\right| \leq-\mu S\left(p_{N}^{n}\right)$ and Lemma 6.1 implies that $S\left(p_{N}^{n}\right)$ converges strongly. Implicitly we also have $\mathbf{v}_{T} \in L^{2}\left(\Gamma_{S}\right)$ since $\mathbf{v} \in H^{1}(\Omega)$. Thus, $\left[\mathbf{v}_{T}, \mathbf{p}_{T}^{n}\right]_{\Gamma_{S}} \rightarrow\left[\mathbf{v}_{T}, \mathbf{p}_{T}\right]_{\Gamma_{S}}$.

\section{Previous results}

From Theorem 6.3 we obtain the following sufficient condition for existence of solutions to problem (5.13):

$$
\left.\begin{array}{l}
\mathbf{w} \in \mathbb{V}_{0} \cap \mathcal{N} \\
\mathbf{p} \text { finite measure on } \Gamma_{C}, p_{N} \leq 0 \\
\mathbf{w} \text { and } \mathbf{p} \text { satisfy (6.23) and (6.24) for some } \varphi
\end{array}\right\} \Rightarrow \mathbf{w}=\mathbf{0}
$$

We obtain from the condition (6.24) on $\mathbf{w}$ and $\mathbf{p}$ that

$$
[\mathbf{w}, \mathbf{p}]_{\Gamma_{S}} \leq\left\langle\mu S\left(p_{N}\right),\left|\mathbf{w}_{T}\right|\right\rangle_{\Gamma_{S}} \leq 0
$$

so the condition (6.23) implies

$$
(L, \mathbf{w})=\langle\mathbf{f}, \mathbf{w}\rangle_{\Omega}+\langle\mathbf{t}, \mathbf{w}\rangle_{\Gamma_{T}}=-[\mathbf{w}, \mathbf{p}]_{\Gamma_{S}}+\alpha\langle\varphi \mathbf{w}, \mathbf{w}\rangle_{\Omega} \geq 0
$$

and thus

$$
\left.\begin{array}{l}
\mathbf{w} \in \mathbb{V}_{0} \cap \mathcal{N} \\
(L, \mathbf{w}) \geq 0
\end{array}\right\} \Rightarrow \mathbf{w}=\mathbf{0}
$$

is also a sufficient condition for the existence of a solution to problem (5.13) according to (6.27). However, (6.28) does not involve the coefficient of friction, and is more restrictive than (6.27). The condition (6.28) is a classical condition proved by Cocou [6] and Jarušek [21]. Cocou considers nonlocal friction laws like (5.13), and Jarušek considers a local friction law with a small coefficient of friction and a contact problem between two elastic bodies. The condition (6.28) was also proved to be sufficient in the frictionless case by Fichera ([14] and [15]).

The geometrical requirement (5.1) is not necessary if (6.28) holds. To see this, let us prove that $\left\|\mathbf{u}^{n}\right\|_{H^{1}(\Omega)}$ is bounded by contradiction. Let us assume $\left\|\mathbf{u}^{n}\right\|_{H^{1}(\Omega)} \rightarrow \infty$. Note that

$$
\left|\left[1, p_{N}^{n}\right]\right| \leq C_{37}\left\|\mathbf{u}^{n}-\mathbf{P} \mathbf{u}^{n}\right\|_{H^{1}(\Omega)}+C_{38}
$$


according to (6.6) and (5.10) for some constants $C_{37}$ and $C_{38}$. This gives

$$
\left|\left[1, p_{N}^{n}\right] /\left\|\mathbf{u}^{n}\right\|_{H^{1}(\Omega)}\right| \leq C_{37}\left\|\mathbf{w}^{n}-\mathbf{P w}^{n}\right\|_{H^{1}(\Omega)}+C_{38} /\left\|\mathbf{u}^{n}\right\|_{H^{1}(\Omega)} \rightarrow 0
$$

and thus

$$
\begin{aligned}
(L, \mathbf{w})= & \lim _{n \rightarrow \infty} \frac{1}{\left\|\mathbf{u}^{n}\right\|_{H^{1}(\Omega)}}\left(L, \mathbf{u}^{n}\right) \\
= & \lim _{n \rightarrow \infty} \frac{1}{\left\|\mathbf{u}^{n}\right\|_{H^{1}(\Omega)}}\left(a\left(\mathbf{u}^{n}, \mathbf{u}^{n}\right)+\varepsilon_{n}\left\langle\varphi \mathbf{u}^{n}, \mathbf{u}^{n}\right\rangle_{\Omega}-\left[\mathbf{u}^{n}, \mathbf{p}^{n}\right]_{\Gamma_{S}}\right) \\
\geq & \lim _{n \rightarrow \infty} \frac{-1}{\left\|\mathbf{u}^{n}\right\|_{H^{1}(\Omega)}}\left[\mathbf{u}^{n}, \mathbf{p}^{n}\right]_{\Gamma_{S}} \\
\geq & \lim _{n \rightarrow \infty} \frac{-1}{\left\|\mathbf{u}^{n}\right\|_{H^{1}(\Omega)}}\left(\left[u_{N}^{n}-g, p_{N}^{n}\right]_{\Gamma_{C}}+\left[g, p_{N}^{n}\right]_{\Gamma_{C}}\right. \\
& \left.+\left[\mathbf{u}_{T}^{n}-\mathbf{u}_{T}^{\mathrm{ref}}, \mathbf{p}_{T}\right]_{\Gamma_{S}}+\left[\mathbf{u}_{T}^{\mathrm{ref}}, \mathbf{p}_{T}\right]_{\Gamma_{S}}\right) \\
\geq & \lim _{n \rightarrow \infty} \frac{-1}{\left\|\mathbf{u}^{n}\right\|_{H^{1}(\Omega)}}\left(\left[g, p_{N}^{n}\right]_{\Gamma_{C}}+\left[\mathbf{u}_{T}^{\mathrm{ref}}, \mathbf{p}_{T}\right]_{\Gamma_{S}}\right)=0
\end{aligned}
$$

and this contradicts (6.28). This proves that $\left\|\mathbf{u}^{n}\right\|_{H^{1}(\Omega)}$ is a bounded sequence, so that we can construct a solution as a weak limit of $\mathbf{u}^{n}$.

\section{Appendix: Some elementary estimates}

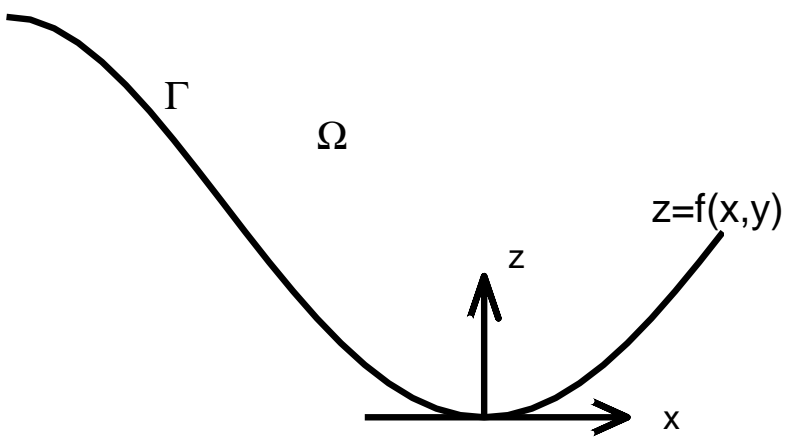

Figure 6.2: Local coordinates. The $\mathrm{z}$-axis is normal to the boundary $\Gamma$.

In this section we derive some estimates, that is Lemma 5.1 and Lemma 5.2. The focus is the three dimensional case.

To prove the first estimate we introduce local coordinates on the boundary. Let $x$ and $y$ be two orthogonal tangential coordinates, and let $z$ denote 
the normal coordinate at some point $\overline{\mathbf{x}} \in \Gamma_{S}$. Let the boundary $\Gamma$ be given by $z=f(x, y)$ close to $\overline{\mathbf{x}} \in \Gamma$, where $f$ is a $C^{2}$ function. Let $\left(x_{q}, y_{q}, z_{q}\right)$ be the local coordinates of the point $\mathbf{q}$ close to $\overline{\mathbf{x}}$.

The approach is to consider some integrals in the tangential xy-plane instead of on the surface $\Gamma$. To have a short notation we define

$$
\begin{aligned}
& \mathbf{x}_{q}=\left(x_{q}, y_{q}, z_{q}\right) \\
& \left|\mathbf{x}_{q}\right|=\sqrt{x_{q}^{2}+y_{q}^{2}+z_{q}^{2}} \\
& \mathbf{x}_{q}^{\prime}=\left(x_{q}, y_{q}, 0\right) \\
& \left|\mathbf{x}_{q}^{\prime}\right|=\sqrt{x_{q}^{2}+y_{q}^{2}} .
\end{aligned}
$$

Since the surface $\Gamma$ is $C^{2}$ we have

$$
\left|z_{q}\right| \leq C_{39}\left|\mathbf{x}_{q}^{\prime}\right|^{2}
$$

for some constant $C_{39}$, and which yields

$$
0 \leq\left|\mathbf{x}_{q}\right|-\left|\mathbf{x}_{q}^{\prime}\right| \leq \frac{1}{2}\left(C_{39}\right)^{2}\left|\mathbf{x}_{q}^{\prime}\right|^{3}
$$

Lemma (5.1). Let

$$
\omega_{\mathbf{x}}(\mathbf{y})=C_{\omega} Q\left(\frac{|\mathbf{x}-\mathbf{y}|}{\rho}\right)= \begin{cases}C_{\omega} e^{\frac{\rho^{2}}{|\mathbf{x}-\mathbf{y}|^{2}-\rho^{2}}} & \text { if }|\mathbf{x}-\mathbf{y}|<\rho \\ 0 & \text { otherwise }\end{cases}
$$

where $|\mathbf{x}-\mathbf{y}|$ is the distance between the two points $\mathbf{x} \in \Gamma_{S}$ and $\mathbf{y} \in \Gamma_{S}$ and $\rho$ and $C_{\omega}$ are constants greater than zero. If $C_{\omega}=C^{*} / \rho^{2}$ where $C^{*}$ is a constant, then it is possible to choose this constant $C^{*}$ so that

$$
\left[\omega_{\overline{\mathbf{x}}}, 1\right]_{\Gamma_{S}}=\int_{\Gamma_{S}} \omega_{\overline{\mathbf{x}}}(\mathbf{y}) \mathrm{d} \Gamma_{\mathbf{y}} \rightarrow 1
$$

uniformly with respect to $\overline{\mathbf{x}} \in \Gamma_{C}$.

Proof. Let

$$
C^{*}=1 / \iint_{\left|\mathbf{x}^{\prime}\right| \leq 1} Q\left(\left|\mathbf{x}^{\prime}\right|\right) \mathrm{d} x \mathrm{~d} y
$$

Taking differential of $\omega$ and using (6.30) we find

$$
\left|Q\left(\frac{\left|\mathbf{x}_{q}\right|}{\rho}\right)-Q\left(\frac{\left|\mathbf{x}_{q}^{\prime}\right|}{\rho}\right)\right| \leq C_{40} \frac{\left|\mathbf{x}_{q}^{\prime}\right|^{3}}{\rho}
$$

where $C_{40}$ is a constant, independent on $\rho$. 
Let us write $f$ instead of $f\left(x_{q}, y_{q}\right), f_{x}^{\prime}$ instead of $f_{x}^{\prime}\left(x_{q}, y_{q}\right)$ and $f_{y}^{\prime}$ instead of $f_{y}^{\prime}\left(x_{q}, y_{q}\right)$. We have $\left|f_{x}^{\prime}\right| \leq C_{41}\left|\mathbf{x}_{q}^{\prime}\right|,\left|f_{y}^{\prime}\right| \leq C_{41}\left|\mathbf{x}_{q}^{\prime}\right|$ and $|f| \leq$ $C_{41}\left|\mathbf{x}_{q}^{\prime}\right|^{2}$ for some constant $C_{41}$ in the local frame of coordinates, so

$$
\left|\sqrt{1+\left(f_{x}^{\prime}\right)^{2}+\left(f_{y}^{\prime}\right)^{2}}-1\right| \leq \frac{1}{2}\left(\left(f_{x}^{\prime}\right)^{2}+\left(f_{y}^{\prime}\right)^{2}\right) \leq C_{41}^{2}\left|\mathbf{x}_{q}^{\prime}\right|^{2}
$$

and thus

$$
\sqrt{1+\left(f_{x}^{\prime}\right)^{2}+\left(f_{y}^{\prime}\right)^{2}}=1+\ell\left(x_{q}, y_{q}\right)\left|\mathbf{x}_{q}^{\prime}\right|^{2}
$$

where $\left|\ell\left(x_{q}, y_{q}\right)\right| \leq C_{41}^{2}$.

Now,

$$
\int_{\Gamma} \omega_{\mathbf{x}}(\mathbf{y}) \mathrm{d} \Gamma_{\mathbf{y}}=\iint_{|\mathbf{x}| \leq \rho} \frac{C^{*}}{\rho^{2}} Q\left(\frac{\left|\mathbf{x}_{q}\right|}{\rho}\right) \sqrt{1+\left(f_{x}^{\prime}\right)^{2}+\left(f_{y}^{\prime}\right)^{2}} \mathrm{~d} x \mathrm{~d} y
$$

so the estimates (6.32) and (6.33) give

$$
\left|\int_{\Gamma} \omega_{\mathbf{x}}(\mathbf{y}) \mathrm{d} \Gamma_{\mathbf{y}}-\iint_{\left|\mathbf{x}^{\prime}\right| \leq \rho} \frac{C^{*}}{\rho^{2}} Q\left(\frac{\left|\mathbf{x}_{q}^{\prime}\right|}{\rho}\right) \mathrm{d} x \mathrm{~d} y\right| \leq C_{42} \rho^{2}
$$

where $C_{42}$ is a constant. But

$$
\iint_{\left|\mathbf{x}^{\prime}\right| \leq \rho} \frac{C^{*}}{\rho^{2}} Q\left(\frac{\left|\mathbf{x}_{q}^{\prime}\right|}{\rho}\right) \mathrm{d} x \mathrm{~d} y=1
$$

by the choice of $C^{*}$, so (6.34) proves the proposition.

Lemma (5.2). Let $h: \mathbb{R}^{3} \rightarrow \mathbb{R}$ denote a Lipschitz function and $\Gamma^{\prime} \subset \Gamma_{C}$. Then, there exist a bounded function, $\Phi(\overline{\mathbf{x}}, \rho)$, such that $\Phi(\overline{\mathbf{x}}, \rho)<\Phi_{\max }<$ $\infty$ for all $\overline{\mathbf{x}} \in \bar{\Gamma}_{C}$, and a constant $C_{9}$ which depends only on the Lipschitz constant of $h$, such that

$$
\left|\left[\omega_{\overline{\mathbf{x}}}, h\right]_{\Gamma^{\prime}}-\Phi(\overline{\mathbf{x}}, \rho) h(\overline{\mathbf{x}})\right| \leq C_{9} \rho .
$$

for all $\overline{\mathbf{x}} \in \bar{\Gamma}_{C}$.

Proof. There is a constant $C_{43}$ such that for any $\overline{\mathbf{x}} \in \Gamma^{\prime}$

$$
\int_{|\mathbf{x}-\overline{\mathbf{x}}| \leq \rho} \mathrm{d} \Gamma_{\mathbf{x}} \leq C_{43} \rho^{2}
$$

that is, the area of the surface $\left\{\mathbf{x} \in \Gamma_{S}:|\mathbf{x}-\overline{\mathbf{x}}| \leq \rho\right\}$ is less than $C_{43} \rho^{2}$. Let $\Phi(\overline{\mathbf{x}}, \rho)=\left[\omega_{\overline{\mathbf{x}}}, 1\right]_{\Gamma^{\prime}}=\int_{\Gamma^{\prime}} \omega_{\overline{\mathbf{x}}}(\mathbf{x}) \mathrm{d} \Gamma_{\mathbf{x}}$, since $\left|\omega_{\overline{\mathbf{x}}}\right| \leq C^{*} e^{-1} / \rho^{2}$ we find

$$
|\Phi(\overline{\mathbf{x}}, \rho)| \leq \frac{C^{*} e^{-1}}{\rho^{2}} C_{43} \rho^{2}
$$


and thus $\Phi$ is bounded.

This choice of $\Phi$ implies

$$
\left|\left[\omega_{\overline{\mathbf{x}}}, h\right]_{\Gamma^{\prime}}-\Phi(\overline{\mathbf{x}}, \rho) h(\overline{\mathbf{x}})\right|=\left|\int_{\Gamma^{\prime}}(h(\mathbf{q})-h(\overline{\mathbf{x}})) \omega_{\overline{\mathbf{x}}}(\mathbf{q}) \mathrm{d} \Gamma_{\mathbf{q}} \cdot\right|
$$

We have $|h(\mathbf{q})-h(\overline{\mathbf{x}})| \leq L \rho$ when $|\mathbf{q}-\overline{\mathbf{x}}| \leq \rho$, where $L$ is the Lipschitz constant of $h(\cdot)$, so that

$$
\begin{aligned}
\left|\left[\omega_{\overline{\mathbf{x}}}, h\right]_{\Gamma^{\prime}}-\Phi(\overline{\mathbf{x}}, \rho) h(\overline{\mathbf{x}})\right| & \leq\left|\int_{\Gamma^{\prime}}(h(\mathbf{q})-h(\overline{\mathbf{x}})) \omega_{\overline{\mathbf{x}}}(\mathbf{q}) \mathrm{d} \Gamma_{\mathbf{q}}\right| \\
& \leq L \rho\left|\int_{\Gamma^{\prime}} \omega_{\overline{\mathbf{x}}}(\mathbf{q}) \mathrm{d} \Gamma_{\mathbf{q}}\right| \\
& \leq L \rho \Phi(\overline{\mathbf{x}}, \rho) \leq L \rho \Phi_{\max }
\end{aligned}
$$




\section{Chapter 7}

\section{Example on nonlocal friction}

In this chapter we consider an example of a friction problem with a nonlocal law of friction. Before studying the example, we prove some auxiliary results.

\section{Some auxiliary results}

To interpret (6.24), we use the following lemma:

7.1. Lemma. The inequality (6.24) implies $p_{N} w_{N}=0,\left|\mathbf{p}_{T}\right| \leq \mu S\left(p_{N}\right)$ and

$$
\left\{\begin{array}{l}
\left|\mathbf{p}_{T}\right|<-\mu S\left(p_{N}\right) \Rightarrow \mathbf{w}_{T}=\mathbf{0} \\
\left|\mathbf{p}_{T}\right|=-\mu S\left(p_{N}\right) \Rightarrow \exists \lambda \geq 0, \mathbf{w}_{T}=-\lambda \mathbf{p}_{T}
\end{array}\right.
$$

in the sense of distributions, where $\lambda \geq 0$.

Proof. Let $\mathbf{v}_{T}=\mathbf{w}_{T}$ and $v_{N}=0$ in (6.24)

$$
[\mathbf{v}-\mathbf{w}, \mathbf{p}]_{\Gamma_{S}}-\left\langle\mu S\left(p_{N}\right),\left|\mathbf{v}_{T}\right|-\left|\mathbf{w}_{T}\right|\right\rangle_{\Gamma_{S}} \geq 0 .
$$

We obtain $\left[w_{N}, p_{N}\right]=0$, and since $p_{N} \leq 0$ and $w_{N} \leq 0$, we find $p_{N} w_{N}=0$ in the sense of distributions.

Since (6.24) holds for all $\mathbf{v} \in\left\{\mathbf{v} \in \mathbb{V}_{0}: \mathbf{v}\right.$ continuous on $\left.\bar{\Gamma}_{C}\right\}$, we have

$$
[\mathbf{v}, \mathbf{p}]_{\Gamma_{S}}-\left\langle\mu S\left(p_{N}\right),\left|\mathbf{v}_{T}\right|\right\rangle_{\Gamma_{S}} \geq 0 .
$$

from which we can deduce $\left|\mathbf{p}_{T}\right| \leq-\mu S\left(p_{N}\right)$.

For any function $\vartheta \in C\left(\Gamma_{S}\right): \Gamma_{S} \rightarrow[0,1]$, we obtain

$$
-\left[\mathbf{w}_{T}, \mathbf{p}_{T} \vartheta\right]_{\Gamma_{S}}+\left\langle\mu S\left(p_{N}\right),\left|\mathbf{w}_{T}\right| \vartheta\right\rangle_{\Gamma_{S}} \geq 0
$$


when $v_{N}=w_{N}$ and $\mathbf{v}_{T}=(1-\vartheta) \mathbf{w}_{T}$ in (6.24). Thus

$$
-\mathbf{p}_{T} \mathbf{w}_{T}+\mu S\left(p_{N}\right)\left|\mathbf{w}_{T}\right| \geq 0
$$

which yields

$$
\left\{\begin{array}{l}
\left|\mathbf{p}_{T}\right|<-\mu(\mathbf{x}) S\left(p_{N}\right) \Rightarrow \mathbf{w}_{T}=\mathbf{0} \\
\left|\mathbf{p}_{T}\right|=-\mu(\mathbf{x}) S\left(p_{N}\right) \Rightarrow \exists \lambda \geq 0, \mathbf{w}_{T}=-\lambda \mathbf{p}_{T} .
\end{array}\right.
$$

since $\left|\mathbf{p}_{T}\right| \leq-\mu S\left(p_{N}(\mathbf{w})\right)$ and $-\mu S\left(p_{N}\right) \geq 0$.

In the proof of existence of solutions to the nonlocal friction problem we assumed $\rho$ to be small. Another reason to require $\rho$ to be small is the following lemma, which is necessary to derive conditions in terms of the applied force.

7.2. Lemma. We have

$$
\left\langle 1, S\left(p_{N}\right)\right\rangle_{\Gamma_{S}} \rightarrow\left[1, p_{N}\right]_{\bar{\Gamma}_{C}}
$$

when $\rho \rightarrow 0$.

Proof. According to Lemma 5.1, $\int_{\Gamma_{S}} \omega_{\mathbf{x}}(\mathbf{y}) \mathrm{d} \Gamma_{\mathbf{y}} \rightarrow 1$ uniformly with respect to $\mathbf{x} \in \bar{\Gamma}_{C}$. Let us also use $\omega_{\mathbf{x}}(\mathbf{y})=\omega_{\mathbf{y}}(\mathbf{x})$ to obtain

$$
\begin{aligned}
\left\langle 1, S\left(p_{N}\right)\right\rangle_{\Gamma_{S}}= & \int_{\Gamma_{S}}\left[\omega_{\mathbf{x}}(\mathbf{y}), p_{N}(\mathbf{y})\right]_{\bar{\Gamma}_{C}} \mathrm{~d} \Gamma_{\mathbf{x}} \\
= & {\left[\int_{\Gamma_{S}} \omega_{\mathbf{y}}(\mathbf{x}) \mathrm{d} \Gamma_{\mathbf{x}}, p_{N}(\mathbf{y})\right]_{\bar{\Gamma}_{C}} } \\
& \rightarrow\left[1, p_{N}\right]_{\bar{\Gamma}_{C}}
\end{aligned}
$$

by the Fubini-Tonelli theorem on integration.

\section{Example: Flat obstacle}

In this example we consider a friction problem for a linearly elastic continuous body. The example is a two-dimensional body on a flat obstacle with constant coefficient of friction $\mu$, compare Figure 7.1. This problem fulfills the requirement (5.1). The objective of this example is to present a simple and nontrivial friction problem with a nonlocal law of friction. Figure 7.1 also introduces a coordinate frame, a contact surface $\Gamma_{C}$ of the body and the points $A, B$ and $O$. $A$ and $B$ are the endpoints of the flat part $\Gamma_{A B}$ of the contact surface, and $O$ is a point at equal distance $L$ from $A$ and $B$ on the contact surface. We assume the coefficient of friction $\mu$ is constant.

Let us define the $\mathrm{z}$-axis perpendicular to the $\mathrm{x}$ - and $\mathrm{y}$-axis so that $\hat{\mathbf{z}}=\hat{\mathbf{x}} \times \hat{\mathbf{y}}$, where $\hat{\mathbf{x}}, \hat{\mathbf{y}}$ and $\hat{\mathbf{z}}$ are unit vectors along the $x$-, $y$ - and $z$-axis. 
Denote a displacement field along the $\mathrm{x}$-axis by $\mathbf{e}^{x}$, a displacement along the $\mathrm{y}$-axis by $\mathbf{e}^{y}$ and a rotation about the point $O$ by $\mathbf{e}^{o}$. Let us normalize these fields so that in each point $\chi, \mathbf{e}_{\chi}^{x}$ and $\mathbf{e}_{\chi}^{y}$ have unit length and $\mathbf{e}_{\chi}^{o}=\hat{\mathbf{z}} \times \mathbf{q}_{\chi}$ where $\mathbf{q}_{\chi}$ is the radius vector of the point $\chi$ relative to the midpoint $O$.

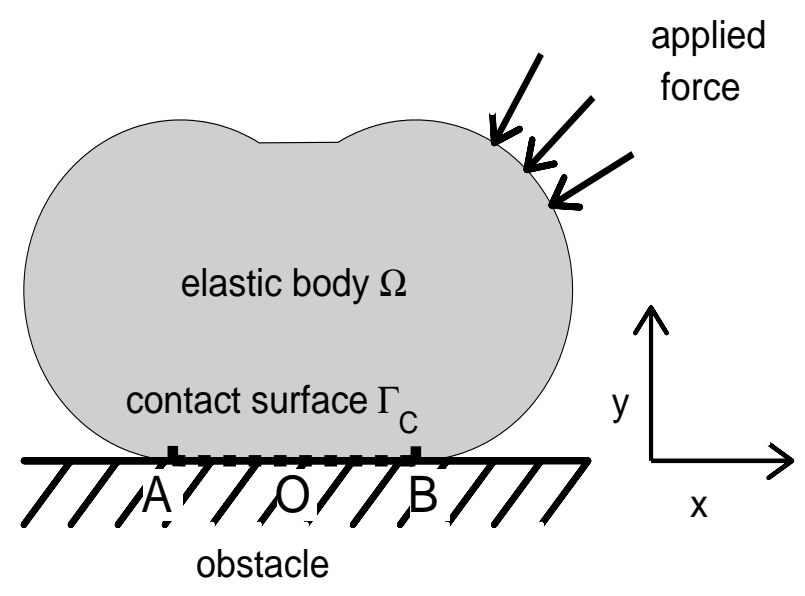

Figure 7.1: A friction problem for a continuous body.

The function $\varphi$ in Theorem 6.3 is now chosen so that $\left\langle\varphi \mathbf{e}^{y}, \mathbf{e}^{o}\right\rangle_{\Omega}=0$, $\left\langle\varphi \mathbf{e}^{x}, \mathbf{e}^{y}\right\rangle_{\Omega}=0$ and $\left\langle\varphi \mathbf{e}^{x}, \mathbf{e}^{o}\right\rangle_{\Omega} \leq 0$. This is possible since a rotation about the point $O$ causes anti-parallel displacements at $A$ and $B$. Denote the torque caused by the applied force about the point $O$ by $M_{o}$, the resultant along the y-axis by $F_{y}$ and the resultant along the x-axis by $F_{x}$. We have

$$
\begin{aligned}
& M_{o}=\left\langle\mathbf{f}, \mathbf{e}^{o}\right\rangle_{\Omega}+\left\langle\mathbf{t}, \mathbf{e}^{o}\right\rangle_{\Gamma_{T}} \\
& F_{x}=\left\langle\mathbf{f}, \mathbf{e}^{x}\right\rangle_{\Omega}+\left\langle\mathbf{t}, \mathbf{e}^{x}\right\rangle_{\Gamma_{T}} \\
& F_{y}=\left\langle\mathbf{f}, \mathbf{e}^{y}\right\rangle_{\Omega}+\left\langle\mathbf{t}, \mathbf{e}^{y}\right\rangle_{\Gamma_{T}} .
\end{aligned}
$$

Let us prove:

7.3. Theorem. There are solutions of the noncoercive friction problem illustrated in Figure 7.1 if

$$
\left\{\begin{aligned}
F_{y} & <0 \\
\left|F_{x}\right|+|\varepsilon| & <-\Lambda \mu F_{y} \\
\left|M_{o}\right|+|\varepsilon| & <-L F_{y}
\end{aligned}\right.
$$

where $\varepsilon \rightarrow 0$ and $\Lambda \rightarrow 1$ when $\rho \rightarrow 0$.

Proof. We decompose $\mathbf{w}$ in components, $\mathbf{w}=w_{x} \mathbf{e}^{x}+w_{y} \mathbf{e}^{y}+w_{o} \mathbf{e}^{o}$. Then, consider the following cases: 
I. The body leaves the contact surface. This means $w_{y}>0$ and $\left|w_{o}\right|<$ $L w_{y}$.

II. $A$ or $B$ will remain in contact. This implies $w_{y}>0$ and $\left|w_{o}\right|=L w_{y}$.

III. Both $A$ and $B$ are in contact, which means $w_{y}=0$ and $w_{o}=0$. Thus $w_{x} \neq 0$, since $\mathbf{w} \neq \mathbf{0}$.

The aim is to prove that the conditions in Theorem (6.3) and the conditions on the applied force (7.2) are incompatible, and then apply Theorem 6.3. Let us consider each case I - III.

I. $\left|w_{N}\right|>0$ on the contact surface, and from Theorem 7.1 follows $p_{N}=0$ and thus $\mathbf{p}=0$. Let $\mathbf{v}=\mathbf{e}^{y}$ in (6.23), then

$$
\begin{aligned}
F_{y} & =\alpha\left\langle\varphi \mathbf{w}, \mathbf{e}^{y}\right\rangle_{\Omega} \\
& =\alpha w_{x}\left\langle\varphi \mathbf{e}^{x}, \mathbf{e}^{y}\right\rangle_{\Omega}+\alpha w_{y}\left\langle\varphi \mathbf{e}^{y}, \mathbf{e}^{y}\right\rangle_{\Omega}+\alpha w_{o}\left\langle\varphi \mathbf{e}^{o}, \mathbf{e}^{y}\right\rangle_{\Omega} \\
& =\alpha w_{y}\left\langle\varphi \mathbf{e}^{y}, \mathbf{e}^{y}\right\rangle_{\Omega} \geq 0
\end{aligned}
$$

by the choice of $\varphi$. This contradicts (7.2).

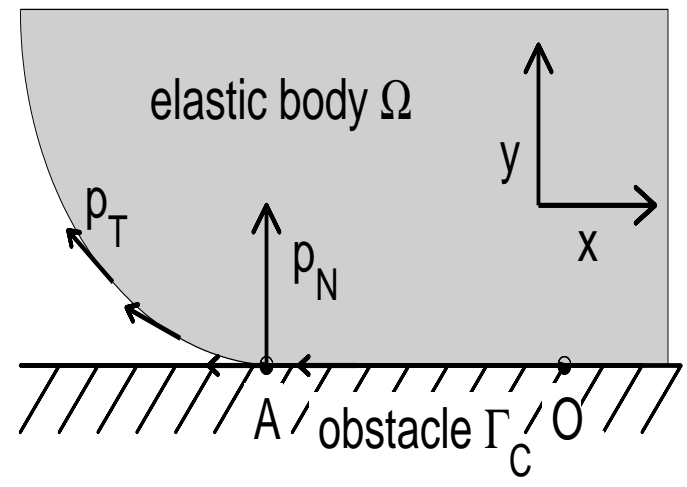

Figure 7.2: Horizontal components of the tangential contact forces due to the curvature of the boundary.

II. Let us assume that $A$ will remain in contact. The case when $B$ remains in contact is similar. Thus $w_{y}>0$ and $w_{o}=\frac{w_{y}}{L}$. Introduce the notation $p_{x}=\left[\mathbf{e}^{x}, \mathbf{p}\right]_{\Gamma_{S}}$ and $p_{y}=\left[\mathbf{e}^{y}, \mathbf{p}\right]_{\Gamma_{S}}$. We have (compare Figure 7.2, and see also [31])

$$
\left\{\begin{array}{l}
\left|p_{x}\right| \geq \Lambda\left[1,\left|\mathbf{p}_{T}\right|\right]_{\Gamma_{S}} \\
p_{y}=\left[1,-p_{N}\right]_{\bar{\Gamma}_{C}}+\varepsilon \\
{\left[\mathbf{e}^{o}, \mathbf{p}\right]_{\Gamma_{S}}=-\mathbf{p}_{y} L+\varepsilon}
\end{array}\right.
$$


where $\Lambda \rightarrow 1$ and $\varepsilon \rightarrow 0$ as $\rho \rightarrow 0$. The origin of $\Lambda$ is the difference between the tangential and $\mathrm{x}$-direction close to the point $A$, and the origin of $\varepsilon$ is $\mathrm{y}$-components of the tangential contact force close to $A$.

Now, let $\mathbf{v}=\mathbf{e}^{x}, \mathbf{v}=\mathbf{e}^{y}$ and $\mathbf{v}=\mathbf{e}^{o}$ in (6.23)

$$
\left\{\begin{aligned}
\mathbf{p}_{x}+F_{x}= & \alpha\left\langle\varphi \mathbf{w}, \mathbf{e}^{x}\right\rangle_{\Omega} \\
& =w_{x} \alpha\left\langle\varphi \mathbf{e}^{x}, \mathbf{e}^{x}\right\rangle_{\Omega}+w_{o} \alpha\left\langle\varphi \mathbf{e}^{o}, \mathbf{e}^{x}\right\rangle_{\Omega} \\
\mathbf{p}_{y}+F_{y}= & \alpha\left\langle\varphi \mathbf{w}, \mathbf{e}^{y}\right\rangle_{\Omega}=w_{y} \alpha\left\langle\varphi \mathbf{e}^{y}, \mathbf{e}^{y}\right\rangle_{\Omega} \\
-\mathbf{p}_{y} L+\varepsilon+M_{o}= & \alpha\left\langle\varphi \mathbf{w}, \mathbf{e}^{o}\right\rangle_{\Omega} \\
& =w_{x} \alpha\left\langle\varphi \mathbf{e}^{x}, \mathbf{e}^{o}\right\rangle_{\Omega}+w_{o} \alpha\left\langle\varphi \mathbf{e}^{o}, \mathbf{e}^{o}\right\rangle_{\Omega}
\end{aligned}\right.
$$

If $w_{x} \leq 0$, then it follows from $(7.3)$

$$
\begin{aligned}
L F_{y}+\varepsilon+M_{o}= & w_{y} L \alpha\left\langle\varphi \mathbf{e}^{y}, \mathbf{e}^{y}\right\rangle_{\Omega}+w_{x} \alpha\left\langle\varphi \mathbf{e}^{x}, \mathbf{e}^{o}\right\rangle_{\Omega} \\
& +w_{o} \alpha\left\langle\varphi \mathbf{e}^{o}, \mathbf{e}^{o}\right\rangle_{\Omega} \geq 0
\end{aligned}
$$

which contradicts (7.2). If $w_{x}>0$, then $\mathbf{p}_{x}=\left[\mathbf{e}^{x}, \mathbf{p}_{T}\right]_{\Gamma_{S}} \leq 0$. Equation (7.4) and Lemma 7.2 give

$$
\begin{aligned}
\mathbf{p}_{x} & \leq-\Lambda\left[1,\left|\mathbf{p}_{T}\right|\right]_{\Gamma_{S}}=-\Lambda \mu\left\langle 1, S\left(p_{N}\right)\right\rangle_{\Gamma_{S}} \\
& \rightarrow-\Lambda \mu\left[1,-p_{N}\right]_{\Gamma_{C}}=-\Lambda \mu\left(\mathbf{p}_{y}-\varepsilon\right)
\end{aligned}
$$

From (7.3) and (7.5) follow

$$
\left\{\begin{aligned}
F_{x}+\Lambda \mu F_{y}+\Lambda \mu \varepsilon \geq & w_{x} \alpha\left\langle\varphi \mathbf{e}^{x}, \mathbf{e}^{x}\right\rangle_{\Omega}+w_{o} \alpha\left\langle\varphi \mathbf{e}^{o}, \mathbf{e}^{x}\right\rangle_{\Omega} \\
& +\Lambda \mu w_{y} \alpha\left\langle\varphi \mathbf{e}^{y}, \mathbf{e}^{y}\right\rangle_{\Omega} \\
L F_{y}+M_{o}+\varepsilon= & L w_{y} \alpha\left\langle\varphi \mathbf{e}^{y}, \mathbf{e}^{y}\right\rangle_{\Omega}+w_{x} \alpha\left\langle\varphi \mathbf{e}^{x}, \mathbf{e}^{o}\right\rangle_{\Omega} \\
& +w_{o} \alpha\left\langle\varphi \mathbf{e}^{o}, \mathbf{e}^{o}\right\rangle_{\Omega}
\end{aligned}\right.
$$

which yields

$$
\begin{aligned}
w_{x}\left(F_{x}+\right. & \left.\Lambda \mu F_{y}+\Lambda \mu \varepsilon\right)+w_{o}\left(L F_{y}+M_{o}+\varepsilon\right) \\
= & \alpha\left(\Lambda \mu w_{x} w_{y}+L w_{o} w_{y}\right)\left\langle\varphi \mathbf{e}^{y}, \mathbf{e}^{y}\right\rangle_{\Omega} \\
& +\alpha\left\langle\varphi\left(w_{o} \mathbf{e}^{o}+w_{x} \mathbf{e}^{x}\right),\left(w_{o} \mathbf{e}^{o}+w_{x} \mathbf{e}^{x}\right)\right\rangle_{\Omega} \geq 0 .
\end{aligned}
$$

We obtain $F_{x}+\Lambda \mu F_{y}+\Lambda \mu \varepsilon>0$ or $L F_{y}+M_{o}+\varepsilon>0$, and thus $\left|F_{x}\right|+|\Lambda \mu \varepsilon|>$ $-\Lambda \mu F_{y}$ or $\left|M_{o}\right|+|\varepsilon|>-L F_{y}$, which is incompatible with (7.2). Note that $\Lambda \rightarrow 1$ and $\Lambda \mu \varepsilon \rightarrow 0$ as $\rho \rightarrow 0$.

III. In this case we obtain from (6.23)

$$
\left\{\begin{array}{l}
\mathbf{p}_{x}+F_{x}=w_{x} \alpha\left\langle\varphi \mathbf{e}^{x}, \mathbf{e}^{x}\right\rangle_{\Omega} \\
\mathbf{p}_{y}+F_{y}=0
\end{array}\right.
$$

Now, we can use $(7.5)$ since $w_{x} \neq 0$ to find

$$
\left|F_{x}\right|=\left|\alpha w_{x}\left\langle\varphi \mathbf{e}^{x}, \mathbf{e}^{x}\right\rangle-\mathbf{p}_{x}\right| \geq\left|\mathbf{p}_{x}\right| \geq \Lambda \mu\left(\mathbf{p}_{y}-\varepsilon\right) \geq-\Lambda \mu\left(F_{y}+\varepsilon\right)
$$

which also contradicts (7.2). 


\section{Part IV}

\section{Continuous Systems with Normal Compliance Friction Laws}





\section{Chapter 8}

\section{Preliminaries}

In this section we study bodies in contact with obstacles where the Signorini non penetration condition is relaxed and replaced by a so called normal compliance law. This means that penetration is possible but penalized by a contact force.

\section{Notation and problem formulation}

We consider an elastic body which in its nondeformed state occupies a domain $\Omega$ in $\mathbb{R}^{2}$ or $\mathbb{R}^{3}$ with a Lipschitz boundary $\Gamma$. Some volume forces $\mathbf{f} \in L^{2}(\Omega)$, surface tractions $\mathbf{t} \in L^{2}(\Gamma)$ and contact forces $\mathbf{p}$ act on the body. The nonzero surface tractions $\mathbf{t}$ act on a part $\Gamma_{T}$ of the boundary $\Gamma$, and the contact forces $\mathbf{p}$ act on a part $\Gamma_{C}$. The partition of the boundary $\Gamma$ is illustrated in Figure 8.1.

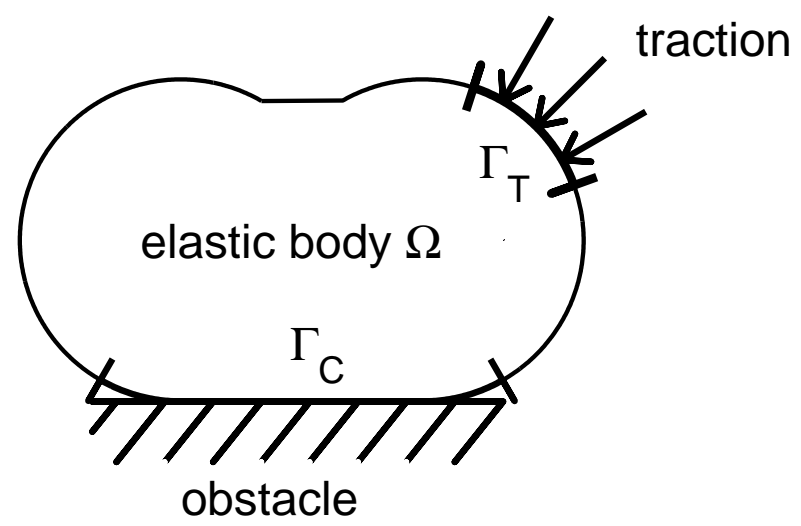

Figure 8.1: The partition of the boundary $\Gamma$. 
$\Gamma: \quad$ The boundary of $\Omega$. Assumed to be Lipschitz.

$\Gamma_{T}$ : Part of $\Gamma$ where the body is subject to nonzero applied tractions.

$\Gamma_{C}$ : Part of $\Gamma$ where the body has possible contact. Assumed to be of $C^{2}$-regularity, so that the normal is continuous.

We also assume that $\Gamma_{T}$ and $\Gamma_{C}$ are relatively open with respect to $\Gamma$, and we denote their closures by $\bar{\Gamma}_{T}$ and $\bar{\Gamma}_{C}$. Moreover, $\bar{\Gamma}_{T}$ and $\bar{\Gamma}_{C}$ are disjoint. Let $\mathbf{n}$ be a normal unit vector on $\Gamma$, directed outwards from $\Omega$.

To study the existence of solutions to the friction problem when $\Omega \subset$ $\mathbb{R}^{3}$, we require that the shape of the contact surface $\Gamma_{C}$ is such that

$$
\left.\begin{array}{c}
\mathbf{v}(\mathbf{x}) \in \mathcal{N} \\
v_{N}(\mathbf{x}) \leq 0 \text { when } \mathbf{x} \in \Gamma_{C}
\end{array}\right\} \Rightarrow \begin{gathered}
\mathbf{v}(\mathbf{x})=\mathbf{0} \text { at most at } \\
\text { one point } \mathbf{x} \text { of } \bar{\Gamma}_{C}
\end{gathered}
$$

where $\mathbf{v}$ is a vector field, $v_{N}=\mathbf{v} \cdot \mathbf{n}$ its normal component on $\Gamma_{C}$ and $\mathcal{N}=\left\{\mathbf{v}: \mathbf{v}=\mathbf{T}+\mathbf{x} \times \mathbf{w}\right.$ where $\left.\mathbf{T}, \mathbf{w} \in \mathbb{R}^{3}\right\}$. In the definition of $\mathcal{N}$ we used $\mathbf{x}$ to denote the radius vector with respect to some origin and $\times$ to denote the cross product.

Geometrically the requirement (8.1) means that if $\mathbf{v}$ is a rotation satisfying $v_{N} \leq 0$ on $\Gamma_{C}$, then the axis of rotation is only allowed to intersect the contact surface $\Gamma_{C}$ once. Such a condition is fulfilled for all two dimensional friction problems, but restrictive in three dimensions, see Chapter 5 section 1. However, the friction problems studied in practice can often be considered as two dimensional due to some symmetry.

Depending on the dimension on the problem we study, let

$$
\mathbb{I}_{d}= \begin{cases}\{1,2\} & \text { for two dimensional problems } \\ \{1,2,3\} & \text { for three dimensional problems }\end{cases}
$$

The equilibrium of force is expressed by

$$
\left\{\begin{array}{c}
\operatorname{div}(\boldsymbol{\sigma})+\mathbf{f}=\mathbf{0} \\
\boldsymbol{\sigma} \cdot \mathbf{n}=\mathbf{t}+\mathbf{p} \text { on } \Gamma
\end{array}\right.
$$

for some stress tensor $\boldsymbol{\sigma}$ with components $\boldsymbol{\sigma}_{i j}$ where $i, j \in \mathbb{I}_{d}$ refer to different coordinate directions in an orthonormal coordinate frame and $\mathbf{n}$ is the normal unit vector which is directed outward from the domain.

Let $\mathbf{u}$ denote the displacement field inside the body. We assume that the body has no support, so that no displacements are prescribed. We also assume the body obeys a linearized law of elasticity,

$$
\boldsymbol{\sigma}=\mathbb{E} \epsilon,
$$

where $\mathbb{E}$ is a tensor of order 4 and the components of the linearized strains $\epsilon$ are

$$
\epsilon_{i j}=\frac{1}{2}\left(\frac{\partial u_{i}}{\partial x_{j}}+\frac{\partial u_{j}}{\partial x_{i}}\right)
$$


where $i, j \in \mathbb{I}_{d}$ and $x_{i}$ is the $i$ :th coordinate. The components $\mathbb{E}_{i j k l}$ of $\mathbb{E}$ fulfill the symmetry conditions $\mathbb{E}_{i j k l}=\mathbb{E}_{j i k l}=\mathbb{E}_{k l i j}$ for all $i, j, k, l \in \mathbb{I}_{d}$. We assume $\mathbb{E}_{i j k l} \in L^{\infty}(\Omega)$ and that these components satisfy the an ellipticity condition

$$
\sum_{i, j, k, l \in \mathbb{I}_{d}} \mathbb{E}_{i j k l} \epsilon_{i j} \epsilon_{k l} \geq C_{7} \sum_{k, l \in \mathbb{I}_{d}} \epsilon_{k l} \epsilon_{k l}
$$

for some constant $C_{7}$.

Let us define the bilinear elastic energy functional

$$
a(\mathbf{u}, \mathbf{v})=\int_{\Omega} \sum_{i, j \in \mathbb{I}_{d}} \epsilon(\mathbf{u})_{i j} \sigma(\mathbf{v})_{i j} \mathrm{~d} \Omega
$$

and the inner products

$$
\begin{aligned}
\langle f, g\rangle_{\Omega} & =\int_{\Omega} f \cdot g \mathrm{~d} \Omega \\
\langle f, g\rangle_{\Gamma} & =\int_{\Gamma} f \cdot g \mathrm{~d} \Gamma
\end{aligned}
$$

and the norms $\|\cdot\|_{L^{2}(\Omega)}$ and $\|\cdot\|_{L^{2}(\Gamma)}$ corresponding to these inner products. By $\mathrm{d} \Omega$ we refer to a measure of volume and by $\mathrm{d} \Gamma$ a measure of surface in three dimensions and a measure of length of the boundary in two dimensions. We also use the Sobolev norm $\|\cdot\|_{H^{1}(\Omega)}$, the supremum norm $\|\cdot\|_{L^{\infty}(\Gamma)}$ and write the duality pairing of $\chi \in H^{1 / 2}(\Gamma)$ and $\psi \in H^{-1 / 2}(\Gamma)$ as $[\chi, \psi]_{\Gamma}$

From the properties of the tensor $\mathbb{E}$ follows that the bilinear elastic energy functional $a(\cdot, \cdot)$ is symmetric, $a(\mathbf{v}, \mathbf{u})=a(\mathbf{u}, \mathbf{v})$ for all $\mathbf{v} \in H^{1}(\Omega)$ and $\mathbf{u} \in H^{1}(\Omega)$, and also that $a(\cdot, \cdot)$ is semicoercive, so that $a(\mathbf{u}, \mathbf{u}) \geq 0$. Moreover,

$$
a(\mathbf{u}, \mathbf{v}) \leq C_{46}\|\mathbf{u}\|_{H^{1}(\Omega)}\|\mathbf{v}\|_{H^{1}(\Omega)}
$$

for some constant $C_{46}$. We have

$$
\begin{aligned}
\mathcal{N} & =\left\{\mathbf{v} \in H^{1}(\Omega): a(\mathbf{v}, \mathbf{w})=0 \text { for every } \mathbf{w} \in H^{1}(\Omega)\right\} \\
& =\left\{\mathbf{v}: \mathbf{v}=\mathbf{T}+\mathbf{x} \times \mathbf{w} \text { where } \mathbf{T}, \mathbf{w} \in \mathbb{R}^{3}\right\}
\end{aligned}
$$

and the set $\mathcal{N}$ is called the set of rigid body displacements. The orthogonal projection map onto $\mathcal{N}$ in $L^{2}(\Omega)$ is denoted by $\mathbf{P}$. The following inequality is proved in [11],

$$
a(\mathbf{v}, \mathbf{v}) \geq C_{47}\|\mathbf{v}-\mathbf{P} \mathbf{v}\|_{H^{1}(\Omega)}^{2}
$$

for all $\mathbf{v} \in H^{1}(\Omega)$ and where $C_{47}>0$. Moreover, the set of rigid body displacements $\mathcal{N}$ is finite dimensional. Thus the $H^{1}$-norm and the $L^{2}$-norm are equivalent on $\mathcal{N}$, so that

$$
C_{48}\|\mathbf{v}\|_{H^{1}(\Omega)} \leq\|\mathbf{v}\|_{L^{2}(\Omega)}
$$


for all $\mathbf{v} \in \mathcal{N}$, where $C_{48}$ is a positive constant, $C_{48}>0$.

In the following we frequently use Green's formula

$$
a(\mathbf{u}, \mathbf{v})+\langle\operatorname{div}(\boldsymbol{\sigma}), \mathbf{v}\rangle_{\Omega}=[\mathbf{v}, \boldsymbol{\sigma} \cdot \mathbf{n}]_{\Gamma} .
$$

Note also that

$$
a\left(\mathbf{u}^{*}, \mathbf{u}^{*}\right) \leq \liminf _{n \rightarrow \infty} a\left(\mathbf{u}^{n}, \mathbf{u}^{n}\right)
$$

if $\mathbf{u}^{n} \rightarrow \mathbf{u}^{*}$ by weak convergence in $H^{1}(\Omega)$, since

$$
\begin{aligned}
2 a\left(\mathbf{u}^{*}, \mathbf{u}^{*}\right) & =2 \liminf _{n \rightarrow \infty} a\left(\mathbf{u}^{*}, \mathbf{u}^{n}\right) \\
& =\liminf _{n \rightarrow \infty}\left(a\left(\mathbf{u}^{*}, \mathbf{u}^{*}\right)+a\left(\mathbf{u}^{n}, \mathbf{u}^{n}\right)-a\left(\mathbf{u}^{*}-\mathbf{u}^{n}, \mathbf{u}^{*}-\mathbf{u}^{n}\right)\right) \\
& \leq a\left(\mathbf{u}^{*}, \mathbf{u}^{*}\right)+\liminf _{n \rightarrow \infty} a\left(\mathbf{u}^{n}, \mathbf{u}^{n}\right) .
\end{aligned}
$$

Also, if $\mathbf{u}^{n} \rightarrow \mathbf{u}^{*}$ by weak convergence in $H^{1}(\Omega)$, then $\mathbf{u}^{n} \rightarrow \mathbf{u}^{*}$ in $L^{2}(\Omega)$, so that

$$
\left\langle\mathbf{u}^{*}, \mathbf{u}^{*}\right\rangle_{\Omega}=\liminf _{n \rightarrow \infty}\left\langle\mathbf{u}^{n}, \mathbf{u}^{n}\right\rangle_{\Omega}
$$

To formulate the laws of contact and friction, we define the normal and tangential components of $\mathbf{u}$ and $\mathbf{p}$,

$$
\begin{array}{ll}
p_{N}=\mathbf{n} \cdot \mathbf{p}, & \mathbf{p}_{T}=\mathbf{p}-p_{N} \mathbf{n}, \\
u_{N}=\mathbf{n} \cdot \mathbf{u}, & \mathbf{u}_{T}=\mathbf{u}-u_{N} \mathbf{n} .
\end{array}
$$

In this section the Signorini impenetrability condition is relaxed and replaced by a so called normal compliance law. This means that penetration of the obstacle is possible but penalized by a contact force

$$
p_{N}=-\kappa\left[u_{N}\right]_{+},
$$

where $\left[u_{N}\right]_{+}=\max \left(0, u_{N}\right)$ and $\kappa>0$ is the coefficient of compliance.

Let $\mu$ denote the coefficient of friction. We require that $\mu$ is continuous on $\bar{\Gamma}_{C}$ and $\mu_{\max } \geq \mu \geq \mu_{\min }>0$ for some constants $\mu_{\min }$ and $\mu_{\max }$. We use an incremental Coulomb law of friction which requires that the contact force satisfies $\left|\mathbf{p}_{T}\right| \leq-\mu p_{N}$ and

$$
\left\{\begin{array}{l}
\left|\mathbf{p}_{T}\right|<-\mu p_{N} \Rightarrow \mathbf{u}_{T}=\mathbf{0} \\
\left|\mathbf{p}_{T}\right|=-\mu p_{N} \Rightarrow \exists \lambda \geq 0, \mathbf{u}_{T}=-\lambda \mathbf{p}_{T}
\end{array}\right.
$$

on $\Gamma_{C}$.

The normal compliance friction problem is defined by (8.2), (8.3), (8.9) and (8.10). The next lemma formulates the problem as a variational inequality. 
8.1. Lemma. If $\mathbf{u}$ satisfies

$$
\begin{aligned}
(L, \mathbf{v}-\mathbf{u}) \leq & a(\mathbf{u}, \mathbf{v}-\mathbf{u})+\kappa\left\langle\left[u_{N}\right]_{+}, v_{N}-u_{N}\right\rangle_{\Gamma_{C}} \\
& +\kappa\left\langle\mu\left[u_{N}\right]_{+},\left|\mathbf{v}_{T}\right|-\left|\mathbf{u}_{T}\right|\right\rangle_{\Gamma_{C}}
\end{aligned}
$$

for all $\mathbf{v} \in \mathbb{V}=\left\{\mathbf{v} \in H^{1}: v_{N} \leq 0\right.$ on $\left.\Gamma_{C}\right\}$, where

$$
\begin{aligned}
a(\mathbf{u}, \mathbf{v}) & =\int_{\Omega} \sum_{i, j \in \mathbb{I}_{d}} \boldsymbol{\epsilon}(\mathbf{u})_{i j} \boldsymbol{\sigma}(\mathbf{v})_{i j} d \Omega \\
(L, \mathbf{v}) & =\langle\mathbf{f}, \mathbf{v}\rangle_{\Omega}+\langle\mathbf{t}, \mathbf{v}\rangle_{\Gamma_{T}}
\end{aligned}
$$

then $\operatorname{div}(\boldsymbol{\sigma})+\mathbf{f}=\mathbf{0}$ in $\Omega$ and

$$
\begin{aligned}
{[\mathbf{v}-\mathbf{u}, \mathbf{p}]_{\Gamma_{C}} \geq } & -\kappa\left\langle\left[u_{N}\right]_{+}, v_{N}-u_{N}\right\rangle_{\Gamma_{C}} \\
& -\kappa\left\langle\mu\left[u_{N}\right]_{+},\left|\mathbf{v}_{T}\right|-\left|\mathbf{u}_{T}\right|\right\rangle_{\Gamma_{C}}
\end{aligned}
$$

for all $\mathbf{v} \in \mathbb{V}$. From (8.12) follows

$$
\begin{aligned}
& p_{N}=-\kappa\left[u_{N}\right]_{+} \\
& \left|\mathbf{p}_{T}\right| \leq-\mu p_{N}
\end{aligned}
$$

on the contact surface $\Gamma_{C}$ and

$$
\left\{\begin{array}{l}
\left|\mathbf{p}_{T}\right|<-\mu p_{N} \Rightarrow \mathbf{u}_{T}=\mathbf{0} \\
\left|\mathbf{p}_{T}\right|=-\mu p_{N} \Rightarrow \exists \lambda \geq 0, \mathbf{u}_{T}=-\lambda \mathbf{p}_{T}
\end{array}\right.
$$

Proof. From Green's formula (8.6) and (8.11) we obtain

$$
\begin{aligned}
\langle\mathbf{f}, \mathbf{v}-\mathbf{u}\rangle_{\Omega}+\langle\mathbf{t}, \mathbf{v}-\mathbf{u}\rangle_{\Gamma_{T}} \leq & {[\mathbf{v}-\mathbf{u}, \boldsymbol{\sigma} \cdot \mathbf{n}]_{\Gamma}-\langle\operatorname{div}(\boldsymbol{\sigma}), \mathbf{v}-\mathbf{u}\rangle_{\Omega} } \\
& +\kappa\left\langle\left[u_{N}\right]_{+}, v_{N}-u_{N}\right\rangle_{\Gamma_{C}} \\
& +\kappa\left\langle\mu\left[u_{N}\right]_{+},\left|\mathbf{v}_{T}\right|-\left|\mathbf{u}_{T}\right|\right\rangle_{\Gamma_{C}}
\end{aligned}
$$

By chosing $\mathbf{v}$ so that $\mathbf{v}-\mathbf{u}=\mathbf{0}$ on $\Gamma$, we conclude $\langle-\operatorname{div}(\boldsymbol{\sigma}), \mathbf{v}\rangle_{\Omega}=\langle\mathbf{f}, \mathbf{v}\rangle_{\Omega}$ and thus $-\operatorname{div}(\boldsymbol{\sigma})=\mathbf{f}$. We find

$$
\begin{aligned}
\langle\mathbf{t}, \mathbf{v}-\mathbf{u}\rangle_{\Gamma_{T}} \leq & {[\mathbf{v}-\mathbf{u}, \boldsymbol{\sigma} \cdot \mathbf{n}]_{\Gamma}+\kappa\left\langle\left[u_{N}\right]_{+}, v_{N}-u_{N}\right\rangle_{\Gamma_{C}} } \\
& +\kappa\left\langle\mu\left[u_{N}\right]_{+},\left|\mathbf{v}_{T}\right|-\left|\mathbf{u}_{T}\right|\right\rangle_{\Gamma_{C}}
\end{aligned}
$$

This gives $\boldsymbol{\sigma} \cdot \mathbf{n}=\mathbf{t}$ on $\Gamma_{T}$. On the contact surface $\Gamma_{C}$ we have by definition $\boldsymbol{\sigma} \cdot \mathbf{n}=\mathbf{p}$. Let $\mathbf{v}_{T}=\mathbf{u}_{T}$ in $(8.13)$

$$
\left[v_{N}-u_{N}, p_{N}\right]_{\bar{\Gamma}_{C}}+\kappa\left\langle\left[u_{N}\right]_{+}, v_{N}-u_{N}\right\rangle_{\Gamma_{C}}=0
$$

which yields $p_{N}=-\kappa\left[u_{N}\right]_{+}$on $\Gamma_{C}$. In the same way, we find $(\boldsymbol{\sigma} \cdot \mathbf{n})_{N}=0$ outside $\bar{\Gamma}_{C}$ and $\bar{\Gamma}_{T}$ according to (8.13). We obtain from (8.13)

$$
\left[\mathbf{v}_{T}-\mathbf{u}_{T}, \mathbf{p}_{T}\right]_{\bar{\Gamma}_{C}}+\kappa\left\langle\mu\left[u_{N}\right]_{+},\left|\mathbf{v}_{T}\right|-\left|\mathbf{u}_{T}\right|\right\rangle_{\Gamma_{C}} \geq 0
$$

and from this equation and $p_{N}=-\kappa\left[u_{N}\right]_{+}$we conclude the remaining statements of the proposition. 
In the following, we write Green's formula

$$
a(\mathbf{u}, \mathbf{v}-\mathbf{u})+\langle\operatorname{div}(\boldsymbol{\sigma}), \mathbf{v}-\mathbf{u}\rangle_{\Omega}=[\mathbf{v}-\mathbf{u}, \mathbf{p}]_{\bar{\Gamma}_{C}}+\langle\mathbf{t}, \mathbf{v}-\mathbf{u}\rangle_{\Gamma_{T}}
$$

since $\boldsymbol{\sigma} \cdot \mathbf{n}=\mathbf{t}$ on $\Gamma_{T}$ and $\boldsymbol{\sigma} \cdot \mathbf{n}=\mathbf{p}$ on $\bar{\Gamma}_{C}$. 


\section{Chapter 9}

\section{Existence results for normal compliance friction laws}

This chapter contains existence results for the continuous friction problem with a normal compliance friction law.

\section{Auxiliary friction problem}

The bilinear elastic energy functional $a(\cdot, \cdot)$ is called coercive on $\mathcal{B} \subset H^{1}(\Omega)$ if

$$
a(\mathbf{v}, \mathbf{v}) \geq C_{49}\|\mathbf{v}\|_{H^{1}(\Omega)}^{2}
$$

for all $\mathbf{v} \in \mathcal{B}$ and for some constant $C_{49}>0$. Existence of solutions to the friction problem (8.11) in a space $\mathcal{B}$ where the bilinear elastic energy functional is coercive has been proved by Klarbring et. al. in 1988 ([24]). To study the semicoercive case, let us add a perturbation $\varepsilon_{n}\langle\varphi \mathbf{u}, \mathbf{v}-\mathbf{u}\rangle_{\Omega}$ to the variational inequality (8.11) to obtain an auxiliary problem

$$
\begin{aligned}
(L, \mathbf{v}-\mathbf{u}) \leq & \varepsilon_{n}\langle\varphi \mathbf{u}, \mathbf{v}-\mathbf{u}\rangle_{\Omega}+a(\mathbf{u}, \mathbf{v}-\mathbf{u}) \\
& +\kappa\left\langle\left[u_{N}\right]_{+}, v_{N}-u_{N}\right\rangle_{\Gamma_{C}} \\
& +\kappa\left\langle\mu\left[u_{N}\right]_{+},\left|\mathbf{v}_{T}\right|-\left|\mathbf{u}_{T}\right|\right\rangle_{\Gamma_{C}}
\end{aligned}
$$

for all $\mathbf{v} \in \mathbb{V}$ where $\varphi \in L^{\infty}(\Omega)$ fulfills $\varphi_{\max } \geq \varphi \geq \varphi_{\min }>0$ for some constants $\varphi_{\max }$ and $\varphi_{\min }$

Using (8.7) we prove

9.1. Lemma. Let $\varphi \in L^{\infty}(\Omega)$ be a function $\Omega \rightarrow \mathbb{R}$ bounded from below by a positive constant $\varphi_{\min }$ so that $\varphi \geq \varphi_{\min }>0$. Then there exists a 
constant $C_{50}>0$ such that

$$
\langle\varphi \mathbf{u}, \mathbf{u}\rangle_{\Omega}+a(\mathbf{u}, \mathbf{u}) \geq C_{50}\|\mathbf{u}\|_{H^{1}(\Omega)}^{2}
$$

for all $\mathbf{u} \in H^{1}(\Omega)$.

Thus (9.1) has solutions according to the results by Klarbring et. al., [24] section 4 .

Proof. Assume (9.2) is false, then we can construct a sequence $\left\{\mathbf{v}^{n}\right\}_{n=1}^{\infty}$ such that $\left\|\mathbf{v}^{n}\right\|_{H^{1}(\Omega)}=1$ and

$$
\left\langle\varphi \mathbf{v}^{n}, \mathbf{v}^{n}\right\rangle_{\Omega}+a\left(\mathbf{v}^{n}, \mathbf{v}^{n}\right) \rightarrow 0
$$

when $n \rightarrow \infty$. By Alaoglu's theorem, there exists a $\mathbf{v}^{*}$ such that $\mathbf{v}^{n} \rightarrow \mathbf{v}^{*}$ in $H^{1}(\Omega)$. Thus, (8.7) and (8.8) yield $a\left(\mathbf{v}^{*}, \mathbf{v}^{*}\right)=0$ and $\left\langle\mathbf{v}^{*}, \mathbf{v}^{*}\right\rangle_{\Omega}=0$, which implies $\mathbf{v}^{*}=\mathbf{0}$. From (8.4) we obtain $\mathbf{v}^{n}-\mathbf{P} \mathbf{v}^{n} \rightarrow \mathbf{0}$ in $H^{1}(\Omega)$, and we have $\mathbf{P} \mathbf{v}^{n} \rightarrow \mathbf{P v}^{*}=\mathbf{0}$ since $\mathcal{N}$ is finite dimensional. Hence $\mathbf{v}^{n} \rightarrow \mathbf{v}^{*}=\mathbf{0}$ strongly in $H^{1}(\Omega)$, and thus $0=\left\|\mathbf{v}^{*}\right\|_{H^{1}(\Omega)}=\lim _{n \rightarrow \infty}\left\|\mathbf{v}^{n}\right\|_{H^{1}(\Omega)}=1$, so that we have a contradiction.

$/ / /$

From Lemma 9.1 we conclude that (9.1) is a coercive problem when $\varepsilon_{n}>0$. Denote a solution of this problem $\mathbf{u}^{n}$, and the corresponding contact force $\mathbf{p}^{n}$.

Consider a sequence where $\varepsilon_{n} \rightarrow 0$ and $\varepsilon_{n}>0$. If $\left\|\mathbf{u}^{n}\right\|_{H^{1}(\Omega)} \rightarrow \infty$ then define

$$
\mathbf{w}^{n}=\left\{\begin{array}{cl}
\frac{\mathbf{u}^{n}}{\left\|\mathbf{u}^{n}\right\|_{H^{1}(\Omega)}} & \text { if }\left\|\mathbf{u}^{n}\right\|_{H^{1}(\Omega)} \neq 0 \\
\mathbf{0} & \text { if }\left\|\mathbf{u}^{n}\right\|_{H^{1}(\Omega)}=0 .
\end{array}\right.
$$

The following lemma summarizes some of the properties of the solution of the friction problem (9.1).

9.2. Lemma. I. The inequality (9.1) implies

$$
\left[\mathbf{v}, \mathbf{p}^{n}\right]_{\bar{\Gamma}_{C}}=a\left(\mathbf{v}, \mathbf{u}^{n}\right)+\varepsilon_{n}\left\langle\varphi \mathbf{u}^{n}, \mathbf{v}\right\rangle_{\Omega}-\langle\mathbf{f}, \mathbf{v}\rangle_{\Omega}-\langle\mathbf{t}, \mathbf{v}\rangle_{\Gamma_{T}}
$$

for all $\mathbf{v} \in H^{1}(\Omega)$ and the friction law (8.12) holds.

II. The sequence $\varepsilon_{n}\left\|\mathbf{u}^{n}\right\|_{H^{1}(\Omega)}$ is bounded. When $\left\|\mathbf{u}^{n}\right\|_{H^{1}(\Omega)} \rightarrow \infty$, there exists a strongly convergent subsequence $\mathbf{w}^{n} \rightarrow \mathbf{w}$ in $H^{1}(\Omega)$, where $\mathbf{w}$ is a rigid body motion. In fact,

$$
\left\|\mathbf{u}^{n}\right\|_{H^{1}(\Omega)} a\left(\mathbf{w}^{n}, \mathbf{w}^{n}\right) \leq C_{51}
$$

for some constant $C_{51}$. Moreover

$$
w_{N} \leq 0
$$


and

$$
\left\{\begin{aligned}
\left\|\mathbf{w}^{n}-\mathbf{P w}^{n}\right\|_{H^{1}(\Omega)}^{2} & \leq \frac{C_{52}}{\left\|\mathbf{u}^{n}\right\|_{H^{1}(\Omega)}} \\
\left\|\mathbf{p}^{n}\right\|_{L^{2}\left(\Gamma_{C}\right)}^{2} & \leq C_{53}\left\|\mathbf{u}^{n}\right\|_{H^{1}(\Omega)}
\end{aligned}\right.
$$

where $\mathbf{P}$ is the orthogonal projection on $\mathcal{N}$ in $L^{2}(\Omega)$ and $C_{52}$ and $C_{53}$ are constants.

Proof. $\quad$ I. Let $\mathbf{v}$ fulfill $\mathbf{v}-\mathbf{u}^{n}=\mathbf{0}$ on $\Gamma$, then we obtain by (9.1)

$$
\varepsilon_{n}\left\langle\varphi \mathbf{u}^{n}, \mathbf{v}-\mathbf{u}^{n}\right\rangle_{\Omega}+a\left(\mathbf{u}^{n}, \mathbf{v}-\mathbf{u}^{n}\right)=\left\langle\mathbf{f}, \mathbf{v}-\mathbf{u}^{n}\right\rangle_{\Omega} .
$$

Green's formula implies

$$
\begin{aligned}
a\left(\mathbf{u}^{n}, \mathbf{v}^{n}-\mathbf{u}\right)+\left\langle\operatorname{div}(\sigma), \mathbf{v}^{n}-\mathbf{u}\right\rangle_{\Omega} & =\left[\mathbf{v}-\mathbf{u}^{n}, \mathbf{p}\right]+\left\langle\mathbf{t}, \mathbf{v}-\mathbf{u}^{n}\right\rangle_{\Gamma} \\
& =0
\end{aligned}
$$

if $\mathbf{v}-\mathbf{u}^{n}=\mathbf{0}$ on $\Gamma$, so we obtain

$$
\varepsilon_{n}\left\langle\varphi \mathbf{u}^{n}, \mathbf{v}-\mathbf{u}^{n}\right\rangle_{\Omega}+\left\langle-\operatorname{div} \sigma, \mathbf{v}-\mathbf{u}^{n}\right\rangle_{\Omega}=\left\langle\mathbf{f}, \mathbf{v}-\mathbf{u}^{n}\right\rangle_{\Omega} .
$$

This implies $\varepsilon_{n} \varphi \mathbf{u}^{n}-\operatorname{div} \sigma=\mathbf{f}$. For an arbitrary $\mathbf{v} \in H^{1}(\Omega)$ Green's formula is

$$
a\left(\mathbf{u}^{n}, \mathbf{v}\right)+\langle\operatorname{div}(\sigma), \mathbf{v}\rangle_{\Omega}=[\mathbf{v}, \mathbf{p}]_{\bar{\Gamma}_{C}}+\langle\mathbf{t}, \mathbf{v}\rangle_{\Gamma}
$$

and hence we find

$$
a\left(\mathbf{v}, \mathbf{u}^{n}\right)+\left\langle\varepsilon_{n} \varphi \mathbf{u}^{n}-\mathbf{f}, \mathbf{v}\right\rangle_{\Omega}=\left[\mathbf{v}, \mathbf{p}^{n}\right]_{\bar{\Gamma}_{C}}+\langle\mathbf{t}, \mathbf{v}\rangle_{\Gamma}
$$

which yields the required formula (9.3) after some rearrangement. The friction law (8.12) follows from (9.1) and (9.3).

II. This proof is similar to the proof of (III) in Lemma 6.2. If the sequence $\left\|\mathbf{u}^{n}\right\|_{H^{1}(\Omega)}$ is bounded, then clearly $\varepsilon_{n}\left\|\mathbf{u}^{n}\right\|_{H^{1}(\Omega)}$ is bounded. Thus let us assume $\left\|\mathbf{u}^{n}\right\|_{H^{1}(\Omega)} \rightarrow \infty$ for the remainder of this proof.

Since $\left\|\mathbf{w}^{n}\right\|_{H^{1}(\Omega)} \leq 1$, we can use Alaoglu's theorem. Let us assume the sequence $\mathbf{w}^{n}$ converges weakly to $\mathbf{w}$. To prove that $\mathbf{w}$ is a rigid body displacement, let $\mathbf{v}=\mathbf{0}$ in (9.1)

$$
\begin{aligned}
\left(L,-\mathbf{u}^{n}\right) \leq & \varepsilon_{n}\left\langle\varphi \mathbf{u}^{n},-\mathbf{u}^{n}\right\rangle_{\Omega}+a\left(\mathbf{u}^{n},-\mathbf{u}^{n}\right) \\
& +\kappa\left\langle\left[u_{N}\right]_{+},\left(-u_{N}^{n}\right)\right\rangle_{\Gamma_{C}}-\kappa\left\langle\mu\left[u_{N}\right]_{+},\left|\mathbf{u}_{T}^{n}\right|\right\rangle_{\Gamma_{C}}
\end{aligned}
$$

and since $\kappa\left\langle\mu\left[u_{N}^{n}\right]_{+},\left|\mathbf{u}_{T}^{n}\right|\right\rangle_{\Gamma_{C}} \geq 0$,

$$
\begin{aligned}
\left(L, \mathbf{u}^{n}\right) \geq & \varepsilon_{n}\left\langle\varphi \mathbf{u}^{n}, \mathbf{u}^{n}\right\rangle_{\Omega}+a\left(\mathbf{u}^{n}, \mathbf{u}^{n}\right) \\
& +\kappa\left\langle\left[u_{N}^{n}\right]_{+}, u_{N}^{n}\right\rangle_{\Gamma_{C}}
\end{aligned}
$$


Note that all terms on the right hand side are positive, and also that $\left\langle L, \mathbf{u}^{n}\right\rangle \leq C_{51}\left\|\mathbf{u}^{n}\right\|_{H^{1}(\Omega)}$ for some constant $C_{51}<\infty$. We obtain from (9.6)

$$
\left\{\begin{aligned}
\varepsilon_{n} \varphi_{\min }\left\|\mathbf{u}^{n}\right\|_{H^{1}(\Omega)}\left\langle\mathbf{w}^{n}, \mathbf{w}^{n}\right\rangle_{\Omega} & \leq C_{51} \\
\left\|\mathbf{u}^{n}\right\|_{H^{1}(\Omega)} a\left(\mathbf{w}^{n}, \mathbf{w}^{n}\right) & \leq C_{51} \\
\left\|\mathbf{u}^{n}\right\|_{H^{1}(\Omega)} \kappa\left\|\left[w_{N}^{n}\right]_{+}\right\|_{L^{2}\left(\Gamma_{C}\right)}^{2} & \leq C_{51} .
\end{aligned}\right.
$$

This yields $a\left(\mathbf{w}^{n}, \mathbf{w}^{n}\right) \rightarrow 0$ if $\left\|\mathbf{u}^{n}\right\| \rightarrow \infty$, and hence from (8.7) follows

$$
0 \leq a(\mathbf{w}, \mathbf{w}) \leq \lim _{n \rightarrow \infty} a\left(\mathbf{w}^{n}, \mathbf{w}^{n}\right)=0
$$

Thus, from (8.4), $\mathbf{w}=\mathbf{P} \mathbf{w}$ and $\mathbf{w}^{n}-\mathbf{P w}^{n} \rightarrow 0$ in $H^{1}(\Omega)$. Since $\mathcal{N}$ is finite dimensional, we also have $\mathbf{P} \mathbf{w}^{n} \rightarrow \mathbf{P w}$ in $H^{1}(\Omega)$, so that

$$
\mathbf{w}^{n} \rightarrow \mathbf{w} \text { in } H^{1}(\Omega) .
$$

From (9.7) we also obtain

$$
\varepsilon_{n} \varphi_{\text {min }}\left\|\mathbf{u}^{n}\right\|_{H^{1}(\Omega)}\left\|\mathbf{w}^{n}\right\|_{L^{2}(\Omega)}^{2} \leq C_{51} .
$$

We have $\mathbf{w}^{n} \rightarrow \mathbf{w}$ in $H^{1}(\Omega)$ and can apply (8.5) since $\mathbf{w} \in \mathcal{N}$, so that

$$
\left\|\mathbf{w}^{n}\right\|_{L^{2}(\Omega)}^{2} \geq \frac{1}{2}\|\mathbf{w}\|_{L^{2}(\Omega)}^{2} \geq \frac{C_{48}^{2}}{2}\|\mathbf{w}\|_{H^{1}(\Omega)}^{2}=\frac{C_{48}^{2}}{2}
$$

for large $n$. Therefore

$$
\varepsilon_{n}\left\|\mathbf{u}^{n}\right\|_{H^{1}(\Omega)} \leq \frac{2 C_{51}}{C_{48}^{2} \varphi_{\min }}
$$

for large $n$, so that $\varepsilon_{n}\left\|\mathbf{u}^{n}\right\|_{H^{1}(\Omega)}$ is bounded.

To prove $(9.4)$, we obtain $\left\|\left[w_{N}^{n}\right]_{+}\right\|_{L^{2}\left(\Gamma_{C}\right)}^{2} \rightarrow 0$ when $n \rightarrow \infty$ from (9.7), and conclude $\left\|\left[w_{N}\right]_{+}\right\|_{L^{2}\left(\Gamma_{C}\right)}^{2}=0$. Thus $w_{N} \leq 0$.

Finally we prove (9.5). We obtain from (9.7) and (8.4):

$$
C_{47}\left\|\mathbf{w}^{n}-\mathbf{P} \mathbf{w}^{n}\right\|_{H^{1}(\Omega)}^{2} \leq a\left(\mathbf{w}^{n}, \mathbf{w}^{n}\right) \leq \frac{C_{51}}{\left\|\mathbf{u}^{n}\right\|_{H^{1}(\Omega)}}
$$

so that

$$
\left\|\mathbf{w}^{n}-\mathbf{P w}^{n}\right\|_{H^{1}(\Omega)}^{2} \leq \frac{C_{52}}{\left\|\mathbf{u}^{n}\right\|_{H^{1}(\Omega)}}
$$

and from Lemma 8.1 follows

$$
\begin{aligned}
\left\|\mathbf{p}^{n}\right\|_{L^{2}\left(\Gamma_{C}\right)}^{2} & \leq\left(1+\mu_{\max }\right)^{2}\left\|p_{N}^{n}\right\|_{L^{2}\left(\Gamma_{C}\right)}^{2} \\
& \leq\left(1+\mu_{\max }\right)^{2} \kappa^{2}\left\|\left[u_{N}^{n}\right]_{+}\right\|_{L^{2}\left(\Gamma_{C}\right)}^{2} \\
& \leq\left(1+\mu_{\max }\right)^{2} \kappa^{2}\left\|\mathbf{u}^{n}\right\|_{H^{1}(\Omega)}^{2}\left\|\left[w_{N}^{n}\right]_{+}\right\|_{L^{2}\left(\Gamma_{C}\right)}^{2} \\
& \leq\left(1+\mu_{\max }\right)^{2} \kappa^{2} C_{51}\left\|\mathbf{u}^{n}\right\|_{H^{1}(\Omega)} \\
& \leq C_{53}\left\|\mathbf{u}^{n}\right\|_{H^{1}(\Omega)}
\end{aligned}
$$

for some constants $C_{52}$ and $C_{53}$. 


\section{Existence results}

In this section we will prove compatibility conditions for the friction problem (8.11), using the auxiliary problem (9.1). We consider two possibilities for the solution $\mathbf{u}^{n}$ of (9.1), either $\left\|\mathbf{u}^{n}\right\|$ is bounded or else $\left\|\mathbf{u}^{n}\right\| \rightarrow \infty$ for some subsequence. To simplify the notation we assume $\left\|\mathbf{u}^{n}\right\| \rightarrow \infty$ for the entire sequence in the last case.

Before proving the existence results, we prove some auxiliary results.

9.3. Lemma. If $\left\|\mathbf{u}^{n}\right\|_{H^{1}(\Omega)}$ is a bounded sequence, then there exists a solution to the friction problem (8.11).

Proof. The boundedness is sufficient to ensure the weak convergence of some subsequence

$$
\mathbf{u}^{n} \rightarrow \mathbf{u}^{*} \text { in } H^{1}(\Omega)
$$

for some $\mathbf{u}^{*} \in H^{1}(\Omega)$. By the Sobolev trace imbedding theorem ([12] and [3]) the trace $H^{1}(\Omega) \rightarrow L^{2}(\Gamma)$ is a compact operator, thus

$$
\mathbf{u}^{n} \rightarrow \mathbf{u}^{*} \text { in } L^{2}(\Gamma)
$$

by strong convergence. Hence, by (9.1)

$$
\begin{aligned}
\left(L, \mathbf{v}-\mathbf{u}^{n}\right) \leq & \varepsilon_{n}\left\langle\varphi \mathbf{u}^{n}, \mathbf{v}-\mathbf{u}^{n}\right\rangle_{\Omega}+a\left(\mathbf{u}^{n}, \mathbf{v}-\mathbf{u}^{n}\right) \\
& +\kappa\left\langle\left[u_{N}^{n}\right]_{+},\left(v_{N}-u_{N}^{n}\right)\right\rangle_{\Gamma_{C}} \\
& +\kappa\left\langle\mu\left[u_{N}^{n}\right]_{+},\left|\mathbf{v}_{T}\right|-\left|\mathbf{u}_{T}^{n}\right|\right\rangle_{\Gamma_{C}}
\end{aligned}
$$

for all $\mathbf{v} \in \mathbb{V}$, so if $n \rightarrow \infty$ we obtain by (8.7),

$$
\begin{aligned}
\left(L, \mathbf{v}-\mathbf{u}^{*}\right) \leq & a\left(\mathbf{u}^{*}, \mathbf{v}-\mathbf{u}^{*}\right)+\kappa\left\langle\left[u_{N}^{*}\right]_{+},\left(v_{N}-u_{N}^{*}\right)\right\rangle_{\Gamma_{C}} \\
& +\kappa\left\langle\mu\left[u_{N}^{*}\right]_{+},\left|\mathbf{v}_{T}\right|-\left|\mathbf{u}_{T}^{*}\right|\right\rangle_{\Gamma_{C}}
\end{aligned}
$$

for all $\mathbf{v} \in \mathbb{V}$. Thus $\mathbf{u}^{*}$ is a solution to (8.11).

9.4. Lemma. Let us consider the case when $\left\|\mathbf{u}^{n}\right\|_{H^{1}(\Omega)} \rightarrow \infty$.

I. Let us assume that the shape of the contact surface $\Gamma_{C}$ is such that

$$
\left.\begin{array}{c}
\mathbf{v}(\mathbf{x}) \in \mathcal{N} \\
v_{N}(\mathbf{x}) \leq 0 \text { when } \mathbf{x} \in \Gamma_{C}
\end{array}\right\} \Rightarrow \begin{gathered}
\mathbf{v}(\mathbf{x})=\mathbf{0} \text { at most at } \\
\text { one point } \mathbf{x} \text { of } \bar{\Gamma}_{C}
\end{gathered}
$$

Then $\left\langle 1, p_{N}^{n}\right\rangle$ is bounded, and thus the sequence $\mathbf{p}^{n}$ has a weak limit $\mathbf{p}$ in the sense of measures.

II. If $\left\langle 1, p_{N}^{n}\right\rangle$ is bounded, then

$$
\left\langle\left|\mathbf{p}^{n}\right|,\left|\mathbf{w}^{n}-\mathbf{P w}^{n}\right|\right\rangle_{L^{2}\left(\Gamma_{C}\right)} \rightarrow 0 \text { when } n \rightarrow \infty
$$


III. We have

$$
[\mathbf{v}, \mathbf{p}]_{\bar{\Gamma}_{C}}=\alpha\langle\varphi \mathbf{v}, \mathbf{w}\rangle_{\Omega}-\langle\mathbf{f}, \mathbf{v}\rangle_{\Omega}-\langle\mathbf{t}, \mathbf{v}\rangle_{\Gamma}
$$

for all rigid body displacements $\mathbf{v} \in \mathcal{N}$.

$I V$.

$$
[\mathbf{p}, \mathbf{v}-\mathbf{w}]_{\bar{\Gamma}_{C}}+\left[-\mu p_{N},\left|\mathbf{v}_{T}\right|-\left|\mathbf{w}_{T}\right|\right]_{\bar{\Gamma}_{C}} \geq 0
$$

for all $\mathbf{v} \in \mathbb{V}$ such that $\mathbf{v}$ is continuous on $\bar{\Gamma}_{C}$.

Proof. $\quad$ I. First, let us prove

$$
\left\langle\left|\mathbf{w}^{n}-\mathbf{P} \mathbf{w}^{n}\right|,\left|\mathbf{p}^{n}\right|\right\rangle_{L^{2}\left(\Gamma_{C}\right)} \leq C_{54}<\infty
$$

where $\mathbf{P}$ is the projection on $\mathcal{N}$ and $C_{54}$ is a constant. From the trace theorem we have

$$
\begin{aligned}
\left\|\mathbf{w}^{n}-\mathbf{P} \mathbf{w}^{n}\right\|_{L^{2}\left(\Gamma_{C}\right)}^{2} & \leq\left\|\mathbf{w}^{n}-\mathbf{P} \mathbf{w}^{n}\right\|_{L^{2}(\Gamma)}^{2} \\
& \leq C_{55}\left\|\mathbf{w}^{n}-\mathbf{P} \mathbf{w}^{n}\right\|_{H^{1}(\Omega)}^{2},
\end{aligned}
$$

for some constant $C_{55}$, so that the Cauchy-Schwarz inequality and (9.5) give

$$
\begin{aligned}
\left\langle\left|\mathbf{w}^{n}-\mathbf{P} \mathbf{w}^{n}\right|,\left|\mathbf{p}^{n}\right|\right\rangle_{L^{2}\left(\Gamma_{C}\right)}^{2} & \leq C_{55}^{2}\left\|\mathbf{w}^{n}-\mathbf{P} \mathbf{w}^{n}\right\|_{H^{1}(\Omega)}^{2}\left\|\mathbf{p}^{n}\right\|_{L^{2}\left(\Gamma_{C}\right)}^{2} \\
& \leq C_{55}^{2} \frac{C_{52}}{\left\|\mathbf{u}^{n}\right\|_{H^{1}(\Omega)}} C_{53}\left\|\mathbf{u}^{n}\right\|_{H^{1}(\Omega)} \\
& \leq C_{54}^{2}<\infty
\end{aligned}
$$

for some constant $C_{54}, C_{54}>0$. This proves (9.11).

Let $\mathbf{v}=\mathbf{w}^{n}$ in (9.3), from Lemma $9.2 \mathrm{II}$, the trace theorem and $\left\|\mathbf{w}^{n}\right\|_{L^{2}(\Omega)} \leq 1$ we obtain the inequality

$$
\begin{aligned}
\left|\left[\mathbf{w}^{n}, \mathbf{p}^{n}\right]_{\bar{\Gamma}_{C}}\right| \leq & \left\|\mathbf{u}^{n}\right\|_{H^{1}(\Omega)} a\left(\mathbf{w}^{n}, \mathbf{w}^{n}\right)+\varepsilon_{n} \varphi_{\max }\left\|\mathbf{u}^{n}\right\|_{H^{1}(\Omega)} \\
& +\left|\left\langle\mathbf{f}, \mathbf{w}^{n}\right\rangle_{\Omega}\right|+\left|\left\langle\mathbf{t}, \mathbf{w}^{n}\right\rangle_{\Gamma}\right| \leq C_{56}
\end{aligned}
$$

for some constant $C_{56}, C_{56}<\infty$. We conclude that $\left|\left[\mathbf{w}^{n}, \mathbf{p}^{n}\right]_{\bar{\Gamma}_{C}}\right|$ is bounded. Since $\left|\mathbf{w}^{n}\right| \leq\left|w_{N}^{n}\right|+\left|\mathbf{w}_{T}^{n}\right|$ and $\left|\mathbf{p}^{n}\right| \leq\left(1+\mu_{\max }\right)\left|p_{N}^{n}\right|$ we have

$$
\begin{aligned}
\left|\left[\mathbf{w}^{n}, \mathbf{p}^{n}\right]_{\bar{\Gamma}_{C}}\right| & =-\left[w_{N}^{n}, p_{N}^{n}\right]_{\bar{\Gamma}_{C}}-\left[\mathbf{w}_{T}^{n}, \mathbf{p}_{T}^{n}\right]_{\bar{\Gamma}_{C}} \\
& =\left[\left|w_{N}^{n}\right|,\left|p_{N}^{n}\right|\right]_{\bar{\Gamma}_{C}}+\left[\left|\mathbf{w}_{T}^{n}\right|, \mu\left|p_{N}^{n}\right|\right]_{\bar{\Gamma}_{C}} \\
& \geq \min \left(1, \mu_{\min }\right)\left[\left|w_{N}^{n}\right|+\left|\mathbf{w}_{T}^{n}\right|,\left|p_{N}^{n}\right|\right]_{\bar{\Gamma}_{C}} \\
& \geq \frac{\min \left(1, \mu_{\min }\right)}{1+\mu_{\max }}\left[\left|\mathbf{w}^{n}\right|,\left|\mathbf{p}^{n}\right|\right]_{\bar{\Gamma}_{C}}
\end{aligned}
$$


so that

$$
\left[\left|\mathbf{w}^{n}\right|,\left|\mathbf{p}^{n}\right|\right]_{\Gamma_{C}} \leq \frac{1+\mu_{\max }}{\min \left(1, \mu_{\min }\right)}\left|\left[\mathbf{w}^{n}, \mathbf{p}^{n}\right]\right| \leq \frac{\left(1+\mu_{\max }\right) C_{56}}{\min \left(1, \mu_{\min }\right)} .
$$

From (9.11) and (9.12) we deduce

$$
\begin{aligned}
\left|\left[\left|\mathbf{P} \mathbf{w}^{n}\right|,\left|\mathbf{p}^{n}\right|\right]_{\bar{\Gamma}_{C}}\right| & \leq\left[\left|\mathbf{w}^{n}\right|,\left|\mathbf{p}^{n}\right|\right]_{\bar{\Gamma}_{C}}+\left|\left[\left|\mathbf{P} \mathbf{w}^{n}-\mathbf{w}^{n}\right|,\left|\mathbf{p}^{n}\right|\right]_{\bar{\Gamma}_{C}}\right| \\
& \leq \frac{\left(1+\mu_{\max }\right) C_{56}}{\min \left(1, \mu_{\min }\right)}+C_{54}<\infty
\end{aligned}
$$

so that $\left|\left[\left|\mathbf{P} \mathbf{w}^{n}\right|,\left|\mathbf{p}^{n}\right|\right]_{\bar{\Gamma}_{C}}\right| \leq C_{57}$ for some constant $C_{57}$.

To prove that $\left[1,\left|\mathbf{p}^{n}\right|\right]_{\bar{\Gamma}_{C}}$ is bounded, consider first the part of $\bar{\Gamma}_{C}$ where $|\mathbf{w}| \geq \delta>0$ for some $\delta$, that is $\Gamma_{C}^{\delta}=\left\{\mathbf{x} \in \bar{\Gamma}_{C}:|\mathbf{w}(\mathbf{x})| \geq\right.$ $\delta>0\}$. Since $\mathbf{P w}^{n} \rightarrow \mathbf{P w}=\mathbf{w}$ uniformly, there is an $N$ so that $\left|\mathbf{P w}^{n}(\mathbf{x})\right| \geq \delta / 2$ for all $\mathbf{x} \in \Gamma_{C}^{\delta}$ when $n \geq N$. We find

$$
C_{57} \geq\left[\left|\mathbf{P} \mathbf{w}^{n}\right|,\left|\mathbf{p}^{n}\right|\right]_{\Gamma_{C}^{\delta}} \geq \delta / 2\left[1,\left|\mathbf{p}^{n}\right|\right]
$$

so that

$$
\left[1,\left|\mathbf{p}^{n}\right|\right]_{\Gamma_{C}^{\delta}} \leq \frac{2 C_{57}}{\delta}
$$

when $n$ is sufficiently large.

If $\mathbf{w}$ is nonzero everywhere on $\bar{\Gamma}_{C}$, then $(9.13)$ proves that $\left[1, p_{N}^{n}\right]_{\bar{\Gamma}_{C}}$ is bounded. Otherwise, let us assume that $\mathbf{w}(\hat{\mathbf{x}})=0$ for some $\hat{\mathbf{x}} \in \bar{\Gamma}_{C}$. Let us define the set

$$
\Lambda=\left\{\mathbf{x} \in \bar{\Gamma}_{C}:\left|\mathbf{n}_{\hat{\mathbf{x}}}-\mathbf{n}_{\mathbf{x}}\right| \leq \frac{1}{2\left(1+\mu_{\max }\right)}\right\}
$$

Since $\mathbf{w}$ does not vanish on $\bar{\Gamma}_{C} \backslash \Lambda,(9.13)$ proves that $\left[1, p_{N}^{n}\right]_{\bar{\Gamma}_{C} \backslash \Lambda}$ is bounded. Let $\overline{\mathbf{n}}$ denote the displacement field with $\overline{\mathbf{n}}=\mathbf{n}_{\hat{\mathbf{x}}}$ at each point. Then $\overline{\mathbf{n}} \in \mathcal{N}$, so that Greens formula (9.3) yields

$$
\begin{aligned}
{\left[\overline{\mathbf{n}}, \mathbf{p}^{n}\right]_{\Lambda}=} & -\left[\overline{\mathbf{n}}, \mathbf{p}^{n}\right]_{\bar{\Gamma}_{C} \backslash \Lambda}+\varepsilon_{n}\left\langle\varphi \mathbf{u}^{n}, \overline{\mathbf{n}}\right\rangle_{\Omega} \\
& -\langle\mathbf{f}, \overline{\mathbf{n}}\rangle_{\Omega}-\langle\mathbf{t}, \overline{\mathbf{n}}\rangle_{\Gamma}
\end{aligned}
$$

Since all terms on the left hand side are bounded, we conclude that $\left[\overline{\mathbf{n}}, \mathbf{p}^{n}\right]_{\Lambda}$ is bounded, $\left[\overline{\mathbf{n}}, \mathbf{p}^{n}\right]_{\Lambda} \leq C_{58}$ for some constant $C_{58}$.

Now,

$$
\left|\left[1, p_{N}^{n}\right]_{\Lambda}\right|=\left|\left[\mathbf{n}, \mathbf{p}^{n}\right]_{\Lambda}\right| \leq\left[\overline{\mathbf{n}}, \mathbf{p}^{n}\right]_{\Lambda}+\left[|\mathbf{n}-\overline{\mathbf{n}}|,\left|\mathbf{p}^{n}\right|\right]_{\Lambda}
$$

Since $|\mathbf{n}-\overline{\mathbf{n}}| \leq \frac{1}{2\left(1+\mu_{\max }\right)}$ and $\left|\mathbf{p}^{n}\right| \leq-\left(1+\mu_{\max }\right) p_{N}^{n}$ we find

$$
\left|\left[1, p_{N}^{n}\right]_{\Lambda}\right| \leq\left[\overline{\mathbf{n}}, \mathbf{p}_{N}^{n}\right]_{\Lambda}+1 / 2\left|\left[1, p_{N}^{n}\right]_{\Lambda}\right|
$$


that is $\left|\left[1, p_{N}^{n}\right]_{\Lambda}\right| \leq 2\left[\overline{\mathbf{n}}, \mathbf{p}^{n}\right]_{\Lambda} \leq 2 C_{58}$.

We have thus proved that $\left[1, p_{N}^{n}\right]_{\bar{\Gamma}_{C}}=\left[1, p_{N}^{n}\right]_{\bar{\Gamma}_{C} \backslash \Lambda}+\left[1, p_{N}^{n}\right]_{\Lambda}$ is bounded, since both $\left[1, p_{N}^{n}\right]_{\bar{\Gamma}_{C} \backslash \Lambda}$ and $\left[1, p_{N}^{n}\right]_{\Lambda}$ are bounded.

II. Let us first assume $\left\|\mathbf{u}^{n}-\mathbf{P} \mathbf{u}^{n}\right\|_{H^{1}(\Omega)} \rightarrow \infty$. Equation (9.3) yields

$$
a\left(\mathbf{v}, \mathbf{u}^{n}\right)=\left[\mathbf{v}, \mathbf{p}^{n}\right]_{\bar{\Gamma}_{C}}-\varepsilon_{n}\left\langle\varphi \mathbf{u}^{n}, \mathbf{v}\right\rangle_{\Omega}+\langle\mathbf{f}, \mathbf{v}\rangle_{\Omega}+\langle\mathbf{t}, \mathbf{v}\rangle_{\Gamma}
$$

Now, since $a\left(\mathbf{v}, \mathbf{u}^{n}\right)=a\left(\mathbf{v}, \mathbf{u}^{n}-\mathbf{P} \mathbf{u}^{n}\right)$,

$$
\begin{aligned}
a\left(\mathbf{v}, \frac{\mathbf{u}^{n}-\mathbf{P} \mathbf{u}^{n}}{\left\|\mathbf{u}^{n}-\mathbf{P} \mathbf{u}^{n}\right\|_{H^{1}(\Omega)}}\right)= & \frac{1}{\left\|\mathbf{u}^{n}-\mathbf{P} \mathbf{u}^{n}\right\|_{H^{1}(\Omega)}}\left(\left[\mathbf{v}, \mathbf{p}^{n}\right]_{\Gamma_{C}}\right. \\
& \left.-\varepsilon_{n}\left\langle\varphi \mathbf{u}^{n}, \mathbf{v}\right\rangle_{\Omega}+\langle\mathbf{f}, \mathbf{v}\rangle_{\Omega}+\langle\mathbf{t}, \mathbf{v}\rangle_{\Gamma}\right)
\end{aligned}
$$

Let us assume $\mathbf{v}$ is continuous on $\bar{\Gamma}_{C}$, then the boundedness of $\left\langle 1, p_{N}^{n}\right\rangle$ and Lemma 9.2 II yields

$$
a\left(\mathbf{v}, \frac{\mathbf{u}^{n}-\mathbf{P u}^{n}}{\left\|\mathbf{u}^{n}-\mathbf{P} \mathbf{u}^{n}\right\|_{H^{1}(\Omega)}}\right) \rightarrow 0
$$

when $n \rightarrow \infty$.

Thus, let us define $\mathbf{z}^{n}$,

$$
\mathbf{z}^{n}=\frac{\mathbf{u}^{n}-\mathbf{P} \mathbf{u}^{n}}{\left\|\mathbf{u}^{n}-\mathbf{P} \mathbf{u}^{n}\right\|_{H^{1}(\Omega)}}
$$

Then $\left\langle\mathbf{z}^{n}, \mathbf{v}\right\rangle_{\Omega}=0$ for all $\mathbf{v} \in \mathcal{N}$. Since $\left\|\mathbf{z}^{n}\right\|_{H^{1}(\Omega)}=1$ so we can assume weak convergence, $\mathbf{z}^{n} \rightarrow \mathbf{z}^{*}$. But $a\left(\mathbf{v}, \mathbf{z}^{*}\right)=0$ for all $\mathbf{v}$ according to $(9.14)$, so that $\mathbf{z}^{*} \in \mathcal{N}$. This means that

$$
\left\langle\mathbf{z}^{*}, \mathbf{z}^{*}\right\rangle_{\Omega}=\lim _{n \rightarrow \infty}\left\langle\mathbf{z}^{n}, \mathbf{z}^{*}\right\rangle_{\Omega}=0
$$

which implies $\mathbf{z}^{*}=\mathbf{0}$.

By the compactness of the Sobolev Trace Imbedding Theorem, we find that $\mathbf{z}^{n} \rightarrow \mathbf{z}^{*}=\mathbf{0}$ in $L^{2}\left(\Gamma_{C}\right)$, thus

$$
\frac{\left\|\mathbf{u}^{n}-\mathbf{P} \mathbf{u}^{n}\right\|_{L^{2}\left(\Gamma_{C}\right)}}{\left\|\mathbf{u}^{n}-\mathbf{P} \mathbf{u}^{n}\right\|_{H^{1}(\Omega)}}=\left\|\mathbf{z}^{n}\right\|_{L^{2}\left(\Gamma_{C}\right)} \rightarrow 0
$$

To prove II we use the Cauchy-Schwarz inequality, (9.5) and (9.15) to find

$$
\begin{aligned}
\left\langle\left|\mathbf{w}^{n}-\mathbf{P} \mathbf{w}^{n}\right|,\left|\mathbf{p}^{n}\right|\right\rangle_{L^{2}\left(\Gamma_{C}\right)}^{2} & \\
\leq & \left\|\mathbf{z}^{n}\right\|_{L^{2}\left(\Gamma_{C}\right)}^{2}\left\|\mathbf{w}^{n}-\mathbf{P} \mathbf{w}^{n}\right\|_{H^{1}(\Omega)}^{2}\left\|\mathbf{p}^{n}\right\|_{L^{2}\left(\Gamma_{C}\right)}^{2} \\
\leq & \left\|\mathbf{z}^{n}\right\|_{L^{2}\left(\Gamma_{C}\right)}^{2} \frac{C_{52}}{\left\|\mathbf{u}^{n}\right\|_{H^{1}(\Omega)}} C_{53}\left\|\mathbf{u}^{n}\right\|_{H^{1}(\Omega)} \\
& \rightarrow 0
\end{aligned}
$$


when $n \rightarrow \infty$, which proves the proposition if $\left\|\mathbf{u}^{n}-\mathbf{P} \mathbf{u}^{n}\right\|_{H^{1}(\Omega)} \rightarrow \infty$. Let us now assume that $\left\|\mathbf{u}^{n}-\mathbf{P} \mathbf{u}^{n}\right\|_{H^{1}(\Omega)}$ is bounded, so there is a constant $C_{59}$ such that $\left\|\mathbf{u}^{n}-\mathbf{P} \mathbf{u}^{n}\right\|_{H^{1}(\Omega)} \leq C_{59}$. The trace theorem yields

$$
\begin{aligned}
\left\|\mathbf{w}^{n}-\mathbf{P} \mathbf{w}^{n}\right\|_{L^{2}\left(\Gamma_{C}\right)}^{2} & \leq\left\|\mathbf{w}^{n}-\mathbf{P} \mathbf{w}^{n}\right\|_{L^{2}(\Gamma)}^{2} \\
& \leq C_{55}^{2}\left\|\mathbf{w}^{n}-\mathbf{P} \mathbf{w}^{n}\right\|_{H^{1}(\Omega)}^{2} \\
& =C_{55}^{2} \frac{\left\|\mathbf{u}^{n}-\mathbf{P} \mathbf{u}^{n}\right\|_{H^{1}(\Omega)}^{2}}{\left\|\mathbf{u}^{n}\right\|_{H^{1}(\Omega)}^{2}} \leq C_{55}^{2} \frac{C_{59}^{2}}{\left\|\mathbf{u}^{n}\right\|_{H^{1}(\Omega)}^{2}}
\end{aligned}
$$

and thus the Cauchy-Schwarz inequality and (9.5) imply

$$
\begin{aligned}
\left\langle\left|\mathbf{w}^{n}-\mathbf{P} \mathbf{w}^{n}\right|,\right. & \left.\left|\mathbf{p}^{n}\right|\right\rangle_{L^{2}\left(\Gamma_{C}\right)}^{2} \\
\leq & C_{55}{ }^{2}\left\|\mathbf{w}^{n}-\mathbf{P} \mathbf{w}^{n}\right\|_{H^{1}(\Omega)}^{2}\left\|\mathbf{p}^{n}\right\|_{L^{2}\left(\Gamma_{C}\right)}^{2} \\
\leq & C_{55}{ }^{2} \frac{C_{59}^{2}}{\left\|\mathbf{u}^{n}\right\|_{H^{1}(\Omega)}^{2}} C_{53}\left\|\mathbf{u}^{n}\right\|_{H^{1}(\Omega)} \\
& \rightarrow 0 .
\end{aligned}
$$

which completes the proof.

III. Let $\mathbf{v} \in \mathcal{N}$ in (9.3). Since $a\left(\mathbf{v}, \mathbf{u}^{n}\right)=0$ we find

$$
\left[\mathbf{v}, \mathbf{p}^{n}\right]_{\bar{\Gamma}_{C}}=\varepsilon_{n}\left\langle\varphi \mathbf{u}^{n}, \mathbf{v}\right\rangle_{\Omega}-\langle\mathbf{f}, \mathbf{v}\rangle_{\Omega}-\langle\mathbf{t}, \mathbf{v}\rangle_{\Gamma_{T}}
$$

Hence we obtain (9.9) by taking limits $n \rightarrow \infty$.

IV. From (8.12) we obtain

$$
\begin{aligned}
{\left[\mathbf{v}-\mathbf{w}^{n}, \mathbf{p}^{n}\right]_{\bar{\Gamma}_{C}}-\left[\left|\mathbf{v}_{T}\right|-\left|\mathbf{w}_{T}^{n}\right|, \mu p_{N}^{n}\right]_{\bar{\Gamma}_{C}} } & \geq-\kappa\left\langle\left[w_{N}^{n}\right]_{+}, v_{N}-w_{N}^{n}\right\rangle_{\Gamma_{C}} \\
& \geq-\kappa\left\|\left[w_{N}^{n}\right]_{+}\right\|_{L^{2}\left(\Gamma_{C}\right)}^{2}
\end{aligned}
$$

since $p_{N}^{n}=-\kappa\left[u_{N}^{n}\right]_{+}$and $v_{N} \leq 0$ on $\Gamma_{C}$. We have $\left\|\left[w_{N}^{n}\right]_{+}\right\|_{L^{2}\left(\Gamma_{C}\right)}^{2} \rightarrow 0$ by (9.4), $\left[\mathbf{v}, \mathbf{p}^{n}\right]_{\bar{\Gamma}_{C}} \rightarrow[\mathbf{v}, \mathbf{p}]_{\bar{\Gamma}_{C}}$ and $\left[\left|\mathbf{v}_{T}\right|, \mu p_{N}^{n}\right]_{\Gamma_{C}} \rightarrow\left[\left|\mathbf{v}_{T}\right|, \mu p_{N}\right]_{\bar{\Gamma}_{C}}$ since $\mathbf{v}$ is assumed to be continuous on $\bar{\Gamma}_{C}$. Now, (9.10) follows by taking limits if we prove

$$
\limsup _{n \rightarrow \infty}\left[\mathbf{p}^{n}, \mathbf{w}^{n}\right]_{\bar{\Gamma}_{C}} \geq[\mathbf{p}, \mathbf{w}]_{\bar{\Gamma}_{C}}
$$

and

$$
\lim _{n \rightarrow \infty}\left[\mu p_{N}^{n},\left|\mathbf{w}_{T}^{n}\right|\right]_{\bar{\Gamma}_{C}}=\left[\mu p_{N},\left|\mathbf{w}_{T}\right|\right]_{\bar{\Gamma}_{C}} .
$$


First, let $\mathbf{v}=\mathbf{w}^{n}$ in (9.3). We find

$$
\begin{aligned}
{\left[\mathbf{w}^{n}, \mathbf{p}^{n}\right]_{\bar{\Gamma}_{C}}=} & \left\|\mathbf{u}^{n}\right\|_{H^{1}(\Omega)} a\left(\mathbf{w}^{n}, \mathbf{w}^{n}\right)+\varepsilon_{n}\left\langle\varphi \mathbf{u}^{n}, \mathbf{w}^{n}\right\rangle_{\Omega} \\
& -\left\langle\mathbf{f}, \mathbf{w}^{n}\right\rangle_{\Omega}-\left\langle\mathbf{t}, \mathbf{w}^{n}\right\rangle_{\Gamma} \\
\geq & \varepsilon_{n}\left\langle\varphi \mathbf{u}^{n}, \mathbf{w}^{n}\right\rangle_{\Omega}-\left\langle\mathbf{f}, \mathbf{w}^{n}\right\rangle_{\Omega}-\left\langle\mathbf{t}, \mathbf{w}^{n}\right\rangle_{\Gamma_{T}}
\end{aligned}
$$

By taking limits we obtain

$$
\limsup \left[\mathbf{w}^{n}, \mathbf{p}^{n}\right]_{\bar{\Gamma}_{C}} \leq \alpha\langle\varphi \mathbf{w}, \mathbf{w}\rangle_{\Omega}-\langle\mathbf{f}, \mathbf{w}\rangle_{\Omega}-\langle\mathbf{t}, \mathbf{w}\rangle_{\Gamma_{T}} .
$$

From (9.9) we have

$$
\alpha\langle\varphi \mathbf{w}, \mathbf{w}\rangle_{\Omega}-\langle\mathbf{f}, \mathbf{w}\rangle_{\Omega}-\langle\mathbf{t}, \mathbf{w}\rangle_{\Gamma}=[\mathbf{w}, \mathbf{p}]_{\bar{\Gamma}_{C}} .
$$

Thus, from (9.18) and (9.19) we obtain $\lim \sup _{n \rightarrow \infty}\left[\mathbf{w}^{n}, \mathbf{p}^{n}\right]_{\bar{\Gamma}_{C}} \leq$ $[\mathbf{w}, \mathbf{p}]_{\Gamma_{C}}$, which proves $(9.16)$.

Let us prove (9.17). Since $p_{N}^{n} \leq 0$ we have

$$
\begin{aligned}
{\left[\mu p_{N}^{n},\left|\mathbf{w}_{T}^{n}\right|\right]_{\bar{\Gamma}_{C}} \geq } & {\left[\mu p_{N}^{n},\left|\left(\mathbf{P} \mathbf{w}^{n}\right)_{T}\right|\right]_{\bar{\Gamma}_{C}} } \\
& +\left[\mu p_{N}^{n},\left|\left(\mathbf{w}^{n}-\mathbf{P} \mathbf{w}^{n}\right)_{T}\right|\right]_{\bar{\Gamma}_{C}} .
\end{aligned}
$$

We have $\mathbf{P}\left(\mathbf{w}^{n}\right) \rightarrow \mathbf{w}$ uniformly, hence

$$
\lim _{n \rightarrow \infty}\left[\mu p_{N}^{n},\left|\mathbf{P}\left(\mathbf{w}^{n}\right)_{T}\right|\right]_{\bar{\Gamma}_{C}}=\left[\mu p_{N},\left|\mathbf{w}_{T}\right|\right]_{\bar{\Gamma}_{C}} .
$$

Moreover

$$
\left|\left[\mu p_{N}^{n},\left|\left(\mathbf{w}^{n}-\mathbf{P w}^{n}\right)_{T}\right|\right]_{\bar{\Gamma}_{C}}\right| \leq\left\langle\left|\mathbf{p}^{n}\right|,\left|\mathbf{w}^{n}-\mathbf{P w}^{n}\right|\right\rangle_{L^{2}\left(\Gamma_{C}\right)} \rightarrow 0
$$

when $n \rightarrow \infty$ according to (II). Thus, (9.17) follows from (9.20) by taking limits.

The earlier theorems yield the following existence theorem for the friction problems with compliance friction laws:

9.5. Theorem. Lack of solutions to the friction problem (8.11) implies that for every function $\varphi \in L^{\infty}(\Omega)$ such that $\varphi_{\max } \geq \varphi \geq \varphi_{\min }>0$ for some constants $\varphi_{\max }$ and $\varphi_{\min }$ there exist a rigid body displacement $\mathbf{w} \in \mathbb{V}_{0}=\left\{\mathbf{v} \in H^{1}(\Omega): v_{N} \leq 0\right.$ on $\left.\Gamma_{C}\right\}$, a contact force field $\mathbf{p}$ of finite total variation on $\Gamma$ and a constant $\alpha \geq 0$ such that

$I$.

$$
[\mathbf{v}, \mathbf{p}]_{\bar{\Gamma}_{C}}=\alpha\langle\varphi \mathbf{w}, \mathbf{v}\rangle_{\Omega}-\langle\mathbf{f}, \mathbf{v}\rangle_{\Omega}-\langle\mathbf{t}, \mathbf{v}\rangle_{\Gamma_{T}}
$$

for all $\mathbf{v} \in \mathcal{N}$. 
II.

$$
[\mathbf{v}-\mathbf{w}, \mathbf{p}]_{\bar{\Gamma}_{C}}+\left[\left|\mathbf{v}_{T}\right|-\left|\mathbf{w}_{T}\right|,-\mu p_{N}\right]_{\bar{\Gamma}_{C}} \geq 0
$$

for all $\mathbf{v} \in\left\{\mathbf{v} \in \mathbb{V}_{0}: \mathbf{v}\right.$ continuous on $\left.\bar{\Gamma}_{C}\right\}$.

Proof. There are two possibilities when considering the perturbed problem (9.1), either $\left\|\mathbf{u}^{n}\right\|_{H^{1}(\Omega)}$ is a bounded sequence or $\left\|\mathbf{u}^{n}\right\|_{H^{1}(\Omega)} \rightarrow \infty$ for some subsequence. Now, Lemma 9.3 and Lemma 9.4 yield the theorem. 


\section{Chapter 10}

\section{Extensions}

\section{Initial displacement}

Let us consider a more general compliance law friction problem, where

$$
\left\{\begin{array}{l}
p_{N}=-\kappa\left[u_{N}-g\right]_{+} \\
\left|\mathbf{p}_{T}\right| \leq-\mu p_{N} \\
\mathbf{u}_{T}-\mathbf{u}_{T}^{\text {ref }}=-\lambda \mathbf{p}_{T} \text { if }\left|\mathbf{p}_{T}\right|=-\mu p_{N}>0
\end{array}\right.
$$

and $g \in H^{1 / 2}(\Gamma)$ and $\mathbf{u}_{T}^{\text {ref }} \in H^{1}(\Omega)$. The function $g$ represents an initial gap between the body and the obstacle, whereas $\mathbf{u}_{T}^{\text {ref }}$ represents an initial displacement field.

A contact problem for a body which fulfills (10.1) is governed by the inequality

$$
\begin{aligned}
(L, \mathbf{v}-\mathbf{u}) \leq & a(\mathbf{u}, \mathbf{v}-\mathbf{u})+\kappa \int_{\Gamma_{C}}\left[u_{N}-g\right]_{+}\left(v_{N}-u_{N}\right) \mathrm{d} \Gamma \\
& +\kappa \int_{\Gamma_{C}} \mu\left[u_{N}-g\right]_{+}\left(\left|\mathbf{v}_{T}-\mathbf{u}_{T}^{\mathrm{ref}}\right|-\left|\mathbf{u}_{T}-\mathbf{u}_{T}^{\mathrm{ref}}\right|\right) \mathrm{d} \Gamma
\end{aligned}
$$

for all $\mathbf{v} \in\left\{\mathbf{v} \in H^{1}: v_{N} \leq g\right.$ on $\left.\Gamma_{C}\right\}$. The proof that (10.2) yields (10.1) is similar to the proof of Lemma 8.1.

To study (10.2), we change variables so that $u_{N}^{\prime}=u_{N}-g$ and $\mathbf{u}_{T}^{\prime}=$ $\mathbf{u}_{T}-\mathbf{u}_{T}^{\text {ref }}$. Then (10.2) reads

$$
\begin{aligned}
\left(L^{\prime}, \mathbf{v}-\mathbf{u}^{\prime}\right) \leq & a\left(\mathbf{u}^{\prime}, \mathbf{v}-\mathbf{u}^{\prime}\right)+\kappa \int_{\Gamma_{C}}\left[u_{N}^{\prime}\right]_{+}\left(v_{N}-u_{N}^{\prime}\right) \mathrm{d} \Gamma \\
& +\kappa \int_{\Gamma_{C}} \mu\left[u_{N}^{\prime}\right]_{+}\left(\left|\mathbf{v}_{T}\right|-\left|\mathbf{u}_{T}^{\prime}\right|\right) \mathrm{d} \Gamma
\end{aligned}
$$

for all $\mathbf{v} \in\left\{\mathbf{v} \in H^{1}: v_{N} \leq 0\right.$ on $\left.\Gamma_{C}\right\}$, where

$$
\left(L^{\prime}, \mathbf{v}\right)=(L, \mathbf{v})-a(\overline{\mathbf{v}}, \mathbf{v})
$$


and $\overline{\mathbf{v}} \in H^{1}(\Omega)$ is such that $\bar{v}_{N}=g$ and $\overline{\mathbf{v}}_{T}=\mathbf{u}_{T}^{\text {ref }}$ on $\Gamma_{C}$. Let us also introduce a force $\mathbf{f}^{\prime}$

$$
\left(\mathbf{f}^{\prime}, \mathbf{v}\right)=(\mathbf{f}, \mathbf{v})-a(\overline{\mathbf{v}}, \mathbf{v})
$$

so that $\left(L^{\prime}, \mathbf{v}\right)=\left\langle\mathbf{f}^{\prime}, \mathbf{v}\right\rangle_{\Omega}-\langle\mathbf{t}, \mathbf{v}\rangle_{\Gamma_{C}}$. Formally, (10.3) is equivalently to (8.11), thus we conclude that the existence theorem 9.5 holds. Since $\left(\mathbf{f}^{\prime}, \mathbf{v}\right)=$ $(\mathbf{f}, \mathbf{v})$ for all $\mathbf{v} \in \mathcal{N}$, we find that $\mathbf{u}_{T}^{\text {ref }}$ and $g$ have no effect on the compatibility conditions on the applied force $\mathbf{f}$.

\section{Discrete friction problems with compliance friction laws}

Let us consider $n$ nodes attached by springs, that is a truss. Let the displacement of node $i$ be denoted by $\mathbf{u}_{i}$. We consider the following friction problem with a compliance friction law:

$$
\begin{cases}\mathbf{K u}=\mathbf{f}+\mathbf{r} & \\ \mathbf{r}_{i}=\mathbf{0} & \text { when } i \notin I_{C} \\ r_{i, N}=-\kappa\left[u_{i, N}\right]_{+} & \text {when } i \in I_{C} \\ \mathbf{u}_{i, T}=\mathbf{0} & \text { if }\left|\mathbf{r}_{i, T}\right|<-\mu_{i} r_{i, N} \\ \mathbf{u}_{i, T}=\lambda_{i} \mathbf{r}_{i, T} & \text { if }\left|\mathbf{r}_{i, T}\right|=-\mu_{i} r_{i, N}>0\end{cases}
$$

where $\mathbf{f}$ is a block vector composed by the applied forces $\mathbf{f}_{i}$ that act in each node $i, \mathbf{r}$ is a block vector composed by the contact forces $\mathbf{r}_{i}$ that act in each node $i, \mu_{i}$ is the coefficient of friction and $\lambda_{i} \geq 0$. Here, $\mathbf{u}_{i, N}$ and $\mathbf{u}_{i, T}$ are the normal and tangential component of $\mathbf{u}_{i}$ and $\mathbf{r}_{i, N}$ and $\mathbf{r}_{i, T}$ are the normal and tangential component of $\mathbf{r}_{i}$ with respect to some obstacle. The matrix $\mathbf{K}$ is the stiffness matrix and $I_{C}$ is the set of indices of the contact nodes.

This friction problem can be studied as the discrete friction problem (1.5) considered in Chapter 2. The existence theorem reads like Theorem 2.3. We only need to comment on Lemma 2.2 (III). To prove such a property for a problem with a compliance friction law we can use $u_{i, N} r_{i, N}=$ $-\left|u_{i, N} \| r_{i, N}\right|$ so that

$$
\begin{aligned}
\mathbf{r}_{i} \cdot \mathbf{u}_{i} & =u_{i, N} r_{i, N}+\mathbf{r}_{i, T} \cdot \mathbf{u}_{i, T} \\
& =-\left|u_{i, N} \| r_{i, N}\right|-\mu_{i}\left|r_{i, N}\right|\left|\mathbf{u}_{i, T}\right| \\
& \leq-\min \left(1, \mu_{\text {min }}\right)\left|r_{i, N}\right|\left(\left|u_{i, N}\right|+\left|\mathbf{u}_{i, T}\right|\right)
\end{aligned}
$$

Thus $\left|\mathbf{u}_{i}\right| \leq\left|u_{i, N}\right|+\left|\mathbf{u}_{i, T}\right|$ and $\left|\mathbf{r}_{i}\right| \leq\left|r_{i, N}\right|+\left|\mathbf{r}_{i, T}\right| \leq\left(1+\max _{i \in I_{C}} \mu_{i}\right)\left|r_{i, N}\right|$ imply

$$
\mathbf{r}_{i} \cdot \mathbf{u}_{i} \leq-\frac{\min \left(1, \mu_{\min }\right)}{1+\max _{i \in I_{C}} \mu_{i}}\left|\mathbf{r}_{i}\right|\left|\mathbf{u}_{i}\right| \leq 0
$$


which is an inequality of the type (2.8) that is used to prove Lemma 2.2 (III).

All the conclusions and examples in Chapter 3 also apply for discrete noncoercive friction problems with compliance friction laws. 


\section{Part V}

\section{Concluding remarks}





\section{Conclusions and future research}

The aim of this thesis was to study noncoercive friction problems, and our main objective was to determine when these problems have solutions. We considered a quasistatic incremental friction model of Coulomb type.

For discrete friction problems we derived a compatibility condition on the applied force that is sufficient for the existence of a solution. We also interpreted this condition mechanically, and considered some nontrivial examples where this condition was applicable. We found that if the compatibility condition on the applied force is violated, then there is a solution to the corresponding dynamical problem where the object is in motion. This indicates that the quasistatic assumption should be questioned if the condition is not fulfilled.

Moreover, we were able to determine similar conditions for the continuous friction problems with a nonlocal friction law and a normal compliance friction law. In both cases we had to require that the contact surface fulfilled a geometrical constraint, and also that the contact surface was $C^{2}$. However, this restriction on the geometry is always fulfilled for two dimensional friction problems.

This thesis also proposes a way to construct solutions to the noncoercive friction problem. We proved that if some compatibility conditions were satisfied, then we can construct a solution as a limit of a subsequence of solutions to an auxiliary coercive friction problem.

A future task is to discard the incremental formulation. The incremental formulation replaces derivatives with differences of successive displacements, and it would be of interest to develop theory for quasistatic friction problems without this approximation. Also, it would be of interest to consider noncoercive friction problems for continuous elastic bodies without regularized friction laws. Another task is to prove results on existence of solutions when the coefficient of friction is allowed to be zero on some parts of the contact surface. Moreover, noncoercive friction problems may also be interesting to study assuming large displacements, instead of the linearized small displacement theory as used in this thesis. 


\section{Bibliography}

[1] L. E. Andersson, Quasistatic frictional contact problems with finitely many degrees of freedom, LiTH-MAT-R-1999-22, Linköping University, 1999

[2] P. Ballard, A counter-example to uniqueness in quasi-static elastic contact problems with small friction, Int. J. Engrg Sci. 37, 1999, pp. $163-178$

[3] R. Biezuner, Best constants in Sobolev trace inequalities, Nonlinear Analysis, 54, 2003, pp 575-589

[4] G. Bonfanti, A noncoercive friction problem with tangential applied forces in three dimensions, Boll. Un. Mat. Ital. 7, 1993, pp. 149-165

[5] H. Brezis, L. Nirenberg, G. Stampacchia, A remark on Ky Fan's minimax principle, Boll. Un. Mat. Ital. 6, 1972, pp. 293-300

[6] M. Cocu, Existence of solutions of signorini problems with friction, Int. J. Engrg. Sci. 22, 1984, pp. 567-575

[7] J. W. Daniel, Stability of the solution of definite quadratic programs, Math. Prog. 5, 1973, pp. 41-53

[8] L. Demkowicz, J.T. Oden, On some existence and uniqueness results in contact problems with nonlocal friction, Nonlinear Analysis 6, 1982, pp. 1075-1093

[9] G. Duvaut, Équilibre d'un solide élastique avec contact unilatéral et frottement de Coulomb, C.R. Acad. Sci. Paris 290A, 1980, pp. 263265

[10] G. Duvaut, J. L. Lions, Les inéquations en mécanique et en physique, Dunod, Paris, 1972

[11] G. Duvaut, J. L. Lions, Inequalities in Mechanics and Physics, Springer, Berlin, 1976

[12] L. C. Evans, R. F. Gariepy, Measure Theory and Fine Properties of Functions, CRC Press, Boca Raton, 1992. 
[13] K. Fan, A minimax inequality and applications, in: Shisha (Ed.), Inequalities III, Academic Press, New York, 1972, pp. 103-113

[14] G. Fichera, Existence theorems in elasticity, in: Handbuch der Physik, vol VI a/2, Springer, Berlin, 1972, pp. 347-389

[15] G. Fichera, Boundary value problems of elasticity with unilateral constraints, in: Handbuch der Physik, vol VI a/2, Springer, Berlin, 1972, pp. 391-424

[16] H. Goldstein, Classical Mechanics, 2:nd ed, Addison-Wesley, Massachusetts, 1980

[17] R. Hassani, P. Hild, I. Ionescu, Sufficient conditions of non-uniqueness for the Coulomb friction problem, Math. Methods Appl. Sci. 27, 2004, pp. $47-67$

[18] P. Hild, N. Sakki, On solution multiplicity in friction problems with normal compliance, J. Math. An. Appl. 297, 2004, pp. 1-13

[19] D. Hilding, The equilibrium state of a structure subject to frictional contact, Eur. J. Mech. A/Solids, 19, 2000, pp. 1029-1040

[20] J. Jarušek, Contact problems with bounded friction. Coercive case. Czechoslovak Math. J. 33, 1983, pp. 237-261

[21] J. Jarušek, Contact problems with bounded friction. Semicoercive case. Czechoslovak Math. J. 34, 1984, pp. 619-629

[22] N. Kikuchi, J. T. Oden, Contact problems in elasticity: A study of variational inequalities and finite element methods, SIAM, Philadelphia, 1988

[23] A. Klarbring, Examples of non-uniqueness and non-existence of solutions to quasistatic contact problems with friction, Ingen. Achiv. 60, 1990, pp. 529-541

[24] A. Klarbring, A. Mikelic, M. Schillor, Frictional contact problems with normal compliance, Internat. J. Engrg Sci. 26, 1988, pp. 811-832

[25] A. Klarbring, J. Pang, Existence of solutions to discrete semicoercive frictional contact problems, SIAM J. Optim. 8, 1998, pp. 414-442

[26] C. Knudsen, R. Feldberg, H True, Bifurcation and chaos in a model of a rolling wheelsheet, Phil. Trans. R. Soc. Lond. 338, 1992, pp. 455-469

[27] O. Lundvall, A. Klarbring, Simulation of Wear by use of a Non-smooth Newton Method - A Spur Gear Application, Mechanics of Structures and Machines 29, 2001, pp. 223-238 
[28] J. Necas, J. Jarušsek, J. Haslinger, On the solution of the variational inequality to the Signorini problem with small friction, Boll. Un. Mat. Ital. B. 17, 1980, pp. 796-811

[29] J. R. Rice, Y. Ben-Zion, Slip complexity in earthquake fault models, Proc. Nat. Acad. Sci. USA 93, 1996, pp. 3811-3818

[30] A. Rietz, Existence results for noncoercive incremental contact problems with Coulomb friction, Licentiate thesis, LiU-TEK-LIC2002:45, Linköping University, 2002

[31] A. Rietz, Existence results for two dimensional noncoercive incremental friction problems with a nonlocal friction, LITH-MAT-R-2003-30, Linköping University, 2003

[32] W. Rudin, Functional analysis, 2:nd ed, McGraw-Hill, New York, 1991

[33] Yun-Bo Yi, Shuqin Du, J.R.Barber, J.W.Fash, Effect of geometry on thermoelastic instability in disk brakes and clutches, ASME J.Tribology 121, 1999, pp. 661-666. 